\title{
CYCLOTOMIC CARTER-PAYNE HOMOMORPHISMS
}

\author{
SINÉAD LYLE AND ANDREW MATHAS
}

\begin{abstract}
We construct a new family of homomorphisms between (graded) Specht modules of the quiver Hecke algebras of type $A$. These maps have many similarities with the homomorphisms constructed by Carter and Payne in the special case of the symmetric groups, although the maps that we obtain are both more and less general than these.
\end{abstract}

\section{INTRODUCTION}

The degenerate and non-degenerate cyclotomic Hecke algebras of type $G(\ell, 1, n)$ are an important class of algebras that arise naturally in the representation theory of the groups of Lie type [3], the study of rational Cherednik algebras [14] and the categorification of the irreducible highest weight modules of the affine special linear groups [1, 5].

In the representation theory of non-semisimple algebras the key unsolved problems revolve around computing decomposition multiplicities and Ext ${ }^{n}$-spaces between important classes of modules. For the cyclotomic Hecke algebras $\mathscr{H}_{n}^{\Lambda}$ of type $G(\ell, 1, n)$, significant progress has been made on the decomposition number problem through categorification, however, the calculation of $\mathrm{Ext}^{n}$-spaces for Specht modules remains an open problem.

The simplest Ext ${ }^{n}$-spaces are the hom-spaces and even here very little is known. For example, it was only recently shown by Dodge [12] that the dimension of the hom-spaces between Specht modules of the symmetric groups can be arbitrarily large. This was quite surprising because, prior to Dodge's work, there were no known examples of hom-spaces between Specht modules in odd characteristic which had dimension greater than one. Apart from the work in this paper, the only results on hom-spaces between Specht modules for the cyclotomic Hecke algebras are those contained in the recent work of Corlett [8, 9].

We construct a new family of explicit non-zero homomorphisms between the Specht modules of cyclotomic Hecke algebras. Our results are a cyclotomic generalisation of the famous Carter-Payne Theorem [7] for the symmetric groups. In the special case of the symmetric group we construct many of the homomorphisms described by Carter and Payne. We also construct additional maps which are not Carter-Payne maps.

The main new tool that we use to construct our cyclotomic Carter-Payne homomorphisms is to work in the graded setting and to construct homomorphisms for the

Received by the editors February 18, 2013 and, in revised form, October 22, 2013.

2010 Mathematics Subject Classification. Primary 20C08, 20C30.

Key words and phrases. Cyclotomic Hecke algebras, quiver Hecke algebras, Specht modules, Carter-Payne homomorphisms. 
Specht modules, defined over $\mathbb{Z}$, for the cyclotomic quiver Hecke algebras, or cyclotomic KLR algebras, of type $A$. These algebras are certain $\mathbb{Z}$-graded algebras which, over a field, are isomorphic to the cyclotomic Hecke algebras of type $G(\ell, 1, n)$ by work of Brundan and Kleshchev [4. We develop a number of new tools for working in the graded setting which are likely to be of independent interest; see, for example, $\$ 3.2$

To state our main theorem, let $\mathscr{R}_{n}^{\Lambda}$ be the cyclotomic quiver Hecke algebra of type $A$ over $\mathbb{Z}$ which is determined by the quiver with vertex set $I=\mathbb{Z} / e \mathbb{Z}$, where $e \in\{0,2,3,4, \ldots\}$. For each multipartition $\boldsymbol{\lambda}$ there is a graded Specht module $S^{\boldsymbol{\lambda}}$ which is a $\mathbb{Z}$-free $\mathscr{R}_{n}^{\Lambda}$-module [6]. If $K$ is a field then $S^{\boldsymbol{\lambda}} \otimes_{\mathbb{Z}} K$ is isomorphic to the graded Specht module constructed in [15, 20] and, in turn, this module is a graded lift of the (ungraded) Specht module $\underline{S}^{\hat{\lambda}}$ of the cyclotomic Hecke algebras $\mathscr{H}_{n}^{\Lambda}$ [2,10].

In Definition 3.26 we give a purely combinatorial, but quite technical, condition for when a pair $(\boldsymbol{\lambda}, \boldsymbol{\mu})$ of multipartitions is a cyclotomic Carter-Payne pair. The most important special case is when the multipartition $\boldsymbol{\mu}$ can be obtained from $\boldsymbol{\lambda}$ by moving a horizontal strip of $\gamma$ nodes to an earlier row in the diagram of $\boldsymbol{\lambda}$ without changing their residues, where $\gamma<|I|$. Our main result is the following.

Main Theorem. Suppose that $(\boldsymbol{\lambda}, \boldsymbol{\mu})$ is a cyclotomic Carter-Payne pair, where $\boldsymbol{\lambda}$ and $\boldsymbol{\mu}$ are multipartitions of $n$. Then $\operatorname{Hom}_{\mathscr{R}_{n}^{\Lambda}}\left(S^{\boldsymbol{\lambda}}\langle\delta\rangle, S^{\boldsymbol{\mu}}\right) \neq 0$, where $\delta$ is a positive integer determined by $\boldsymbol{\lambda}$ and $\boldsymbol{\mu}$.

An explicit formula for the integer $\delta$ is given in Theorem 3.28. Applying Brundan and Kleshchev's graded isomorphism theorem [4] we obtain the corresponding result for the cyclotomic Hecke algebra $\mathscr{H}_{n}^{\Lambda}$.

Corollary. Suppose that $\mathscr{H}_{n}^{\Lambda}$ is the cyclotomic Hecke algebra defined over a field $K$ and that $(\boldsymbol{\lambda}, \boldsymbol{\mu})$ is a cyclotomic Carter-Payne pair. Then $\operatorname{Hom}_{\mathscr{H}_{n}^{\Lambda}}\left(\underline{S}^{\boldsymbol{\lambda}}, \underline{S}^{\boldsymbol{\mu}}\right) \neq 0$.

The Carter-Payne theorem was proved for symmetric groups in 1980. It proved very difficult to generalise this result to the Iwahori-Hecke algebras of the symmetric groups [11,21,23], which is the level one case of the algebras considered here. We take a very different approach to this problem here, using the machinery of the cyclotomic quiver Hecke algebras to construct homomorphisms $S^{\boldsymbol{\lambda}}\langle\delta\rangle \rightarrow S^{\boldsymbol{\mu}}$.

The homomorphisms of our Main Theorem are described explicitly as multiplication by certain polynomials in the KLR generators $y_{n+1}, \ldots, y_{n+\gamma}$, which are nilpotent versions of the classical Jucys-Murphy elements. These polynomials act on a Specht module for $\mathscr{R}_{n+\gamma}^{\Lambda}$, which we consider as an $\mathscr{R}_{n}^{\Lambda}$-module by restriction, and which admits a filtration in which the Specht modules $S^{\boldsymbol{\lambda}}$ and $S^{\boldsymbol{\mu}}$ appear as quotients. It is not very difficult to show that the $\mathscr{R}_{n}^{\Lambda}$-endomorphism that we define on $S^{\nu}$ induces an $\mathscr{R}_{n}^{\Lambda}$-module homomorphism between these Specht modules, however, the real challenge is in showing that our maps are non-zero. Rather than working algebraically, it turns out to be much easier to use a variation of the diagram calculus introduced by Khovanov and Lauda [18. For the uninitiated, it takes some time to get used to these diagrams, but it is worth the effort because they reduce long pages of calculations with relations to a series of diagrams which neatly encode the same information in a more transparent way.

As a gentle introduction to the use of these diagrams we start by proving our Main Theorem in the one node case, which is when $\gamma=1$. This case contains most of the features of the general case, however, its proof is considerably easier both to 
write down and to understand. The basic idea for proving section 1 when $\gamma>1$ is the same as the one node case, however, the general argument requires more sophisticated techniques for working with diagrams and tableaux. For example, in $\S 3.2$ we introduce the notion of stubborn strings which we think will have wider application. Using these ideas we prove our Main Theorem as Theorem 3.28.

As a general rule it is very hard to construct non-trivial homomorphisms between Specht modules and it is even harder to compose such homomorphisms. One advantage of our explicit construction is that we are able to compose some of our cyclotomic Carter-Payne homomorphisms in Theorem 3.35. As a second application we prove a "row removal" theorem for cyclotomic Carter-Payne homomorphisms Theorem 3.37

\section{Cyclotomic Hecke algebras and Specht modules}

The cyclotomic quiver Hecke algebras were introduced for all oriented quivers in a series of papers by Khovanov and Lauda [18, 19] and Rouquier [25. These algebras are certain $\mathbb{Z}$-graded algebras which depend on an oriented quiver.

This section defines the cyclotomic quiver Hecke algebras of type $A$ and recalls the results from the literature that we need. Throughout this paper we work over the ring of integers $\mathbb{Z}$. Hence, by base change, all of our results hold over an arbitrary commutative ring.

2.1. Graded algebras and homomorphisms. In this paper a graded algebra will mean a $\mathbb{Z}$-graded algebra and a graded module will be a $\mathbb{Z}$-graded module. If $A$ is a graded algebra then $\operatorname{Mod}-A$ is the category of finitely generated graded (right) $A$-modules with degree preserving maps. We use the standard notation of graded representation theory. In particular, if $M=\bigoplus_{d \in \mathbb{Z}} M_{d}$ then $m \in M_{d}$ is homogeneous of degree $d=\operatorname{deg} m$. If $n \in \mathbb{Z}$ then $M\langle n\rangle$ is the graded module obtained by shifting the grading on $M$ up by $n$ so that $M\langle n\rangle_{d}=M_{d-n}$.

If $A$ is a graded algebra let $\underline{A}$ be the ungraded algebra obtained by forgetting the grading on $A$. Similarly, $\underline{M}$ is the (ungraded) $\underline{A}$-module obtained by forgetting the grading on the graded $A$-module $M$. It is well known that if $M$ and $N$ are graded $A$-modules then

$$
\operatorname{Hom}_{\underline{A}}(\underline{M}, \underline{N}) \cong \bigoplus_{d \in \mathbb{Z}} \operatorname{Hom}_{A}(M\langle d\rangle, N)
$$

where $\operatorname{Hom}_{A}(M\langle d\rangle, N) \cong \operatorname{Hom}_{A}(M, N\langle-d\rangle)$ is isomorphic to the space of homogeneous $A$-module homomorphisms $f: M \longrightarrow N$ of degree $d$ such that $f\left(M_{z}\right) \subseteq N_{z+d}$, for $z \in \mathbb{Z}$.

2.2. Cyclotomic quiver Hecke algebras. We are now ready to define the cyclotomic quiver Hecke algebras of type $A$.

Recall from the introduction that we have fixed an integer $e \in\{0,2,3,4, \ldots\}$ and that $I=\mathbb{Z} / e \mathbb{Z}$. Let $\Gamma_{e}$ be the oriented quiver with vertex set $I$ and directed edges $i \longrightarrow i-1$, for $i \in I$. (The orientation of $\Gamma_{e}$ is opposite to that used in [4,15].) Thus $\Gamma$ is a quiver of type $A_{\infty}$ if $e=0$ or of type $A_{e-1}^{(1)}$ if $e>0$. The corresponding 
Cartan matrix $\left(a_{i, j}\right)_{i, j \in I}$ is given by

$$
a_{i, j}= \begin{cases}2, & \text { if } i=j, \\ 0, & \text { if } j \neq i, i \pm 1, \\ -1, & \text { if } i \rightarrow j \text { or } i \leftarrow j, \\ -2, & \text { if } i \rightleftarrows j .\end{cases}
$$

(The case $a_{i, j}=-2$ only occurs if $e=2$.) To the quiver $\Gamma_{e}$ we attach the standard Lie theoretic data of simple roots $\left\{\alpha_{i} \mid i \in I\right\}$, fundamental weights $\left\{\Lambda_{i} \mid i \in I\right\}$, the positive weight lattice $P^{+}=\bigoplus_{i \in I} \mathbb{N} \Lambda_{i}$ and positive roots $Q^{+}=\bigoplus_{i \in I} \mathbb{N} \alpha_{i}$ and we let $($, ) be the bilinear form determined by

$$
\left(\alpha_{i}, \alpha_{j}\right)=a_{i j} \quad \text { and } \quad\left(\Lambda_{i}, \alpha_{j}\right)=\delta_{i j}, \quad \text { for } i, j \in I .
$$

More details can be found, for example, in Kac's book [17, Chapter 1].

Let $\mathfrak{S}_{n}$ be the symmetric group on $n$ letters and let $s_{r}=(r, r+1)$, for $1 \leq r<n$, be the simple transpositions of $\mathfrak{S}_{n}$. Then $\mathfrak{S}_{n}$ acts from the left on elements of the set $I^{n}$ by place permutations.

2.2. Definition (Khovanov-Lauda [18, 19], Rouquier [25]). Suppose that $\Lambda \in P^{+}$ and $n \geq 1$. The cyclotomic quiver Hecke algebra $\mathscr{R}_{n}^{\Lambda}=\mathscr{R}_{n}^{\Lambda}(\mathbb{Z})$ of weight $\Lambda$ and type $\Gamma_{e}$ is the unital associative $\mathbb{Z}$-algebra with generators

$$
\left\{\psi_{1}, \ldots, \psi_{n-1}\right\} \cup\left\{y_{1}, \ldots, y_{n}\right\} \cup\left\{e(\mathbf{i}) \mid \mathbf{i} \in I^{n}\right\}
$$

and relations

$$
\begin{aligned}
& y_{1}^{\left(\Lambda, \alpha_{i_{1}}\right)} e(\mathbf{i})=0, \quad e(\mathbf{i}) e(\mathbf{j})=\delta_{\mathbf{i j}} e(\mathbf{i}), \quad \sum_{\mathbf{i} \in I^{n}} e(\mathbf{i})=1, \\
& y_{r} e(\mathbf{i})=e(\mathbf{i}) y_{r}, \quad \psi_{r} e(\mathbf{i})=e\left(s_{r} \cdot \mathbf{i}\right) \psi_{r}, \quad y_{r} y_{s}=y_{s} y_{r},
\end{aligned}
$$

$$
\begin{aligned}
& \psi_{r} y_{r+1} e(\mathbf{i})=\left(y_{r} \psi_{r}+\delta_{i_{r} i_{r+1}}\right) e(\mathbf{i}), \quad y_{r+1} \psi_{r} e(\mathbf{i})=\left(\psi_{r} y_{r}+\delta_{i_{r} i_{r+1}}\right) e(\mathbf{i}), \\
& \psi_{r} y_{s}=y_{s} \psi_{r}, \quad \text { if } s \neq r, r+1 \text {, } \\
& \psi_{r} \psi_{s}=\psi_{s} \psi_{r}, \quad \text { if }|r-s|>1, \\
& \psi_{r}^{2} e(\mathbf{i})= \begin{cases}0, & \text { if } i_{r}=i_{r+1}, \\
\left(y_{r+1}-y_{r}\right) e(\mathbf{i}), & \text { if } i_{r} \rightarrow i_{r+1}, \\
\left(y_{r}-y_{r+1}\right) e(\mathbf{i}), & \text { if } i_{r} \leftarrow i_{r+1}, \\
\left(y_{r+1}-y_{r}\right)\left(y_{r}-y_{r+1}\right) e(\mathbf{i}), & \text { if } i_{r} \rightleftarrows i_{r+1}, \\
e(\mathbf{i}), & \text { otherwise, }\end{cases} \\
& \psi_{r} \psi_{r+1} \psi_{r} e(\mathbf{i})= \begin{cases}\left(\psi_{r+1} \psi_{r} \psi_{r+1}+1\right) e(\mathbf{i}), & \text { if } i_{r}=i_{r+2} \rightarrow i_{r+1}, \\
\left(\psi_{r+1} \psi_{r} \psi_{r+1}-1\right) e(\mathbf{i}), & \text { if } i_{r}=i_{r+2} \leftarrow i_{r+1}, \\
\left(\psi_{r+1} \psi_{r} \psi_{r+1}+y_{r}-2 y_{r+1}+y_{r+2}\right) e(\mathbf{i}), & \text { if } i_{r}=i_{r+2} \rightleftarrows i_{r+1}, \\
\psi_{r+1} \psi_{r} \psi_{r+1} e(\mathbf{i}), & \text { otherwise, }\end{cases}
\end{aligned}
$$

for $\mathbf{i}, \mathbf{j} \in I^{n}$ and all admissible $r$ and $s$. Moreover, $\mathscr{R}_{n}^{\Lambda}$ is naturally $\mathbb{Z}$-graded with degree function determined by

$$
\operatorname{deg} e(\mathbf{i})=0, \quad \operatorname{deg} y_{r}=2 \quad \text { and } \quad \operatorname{deg} \psi_{s} e(\mathbf{i})=-a_{i_{s}, i_{s+1}},
$$

for $1 \leq r \leq n, 1 \leq s<n$ and $\mathbf{i} \in I^{n}$. 
Throughout the paper, we fix a dominant weight $\Lambda \in P^{+}$and an ordered $\ell$ tuple $\boldsymbol{\kappa}=\left(\kappa_{1}, \ldots, \kappa_{\ell}\right) \in \mathbb{Z}^{\ell}$, the multicharge, such that $\ell=\sum_{i \in I}\left(\Lambda, \alpha_{i}\right)$ and $\Lambda=\Lambda_{\kappa_{1}}+\cdots+\Lambda_{\kappa_{\ell}}$. The algebra $\mathscr{R}_{n}^{\Lambda}$ depends only on $\Lambda$ (and on $\Gamma_{e}$ and $n$ ), however, some of the tableau combinatorics which we introduce below will depend on the choice of $\boldsymbol{\kappa}$.

Suppose that $w \in \mathfrak{S}_{n}$. Then $s_{r_{1}} \ldots s_{r_{l}}$ is a reduced expression for $w$, and $w$ has length $\ell(w)=l$, if $l$ is minimal such that $w=s_{r_{1}} \ldots s_{r_{l}}$, for $1 \leq r_{k}<n$. Fix a reduced expression $w=s_{r_{1}} \ldots s_{r_{l}}$ for $w$ and define $\psi_{w}=\psi_{r_{1}} \ldots \psi_{r_{l}}$. In view of (2.6) $\psi_{w}$ usually depends on the chosen reduced expression for $w$.

2.3. Cyclotomic Hecke algebras. Recall that $\Lambda \in P^{+}$and that we have fixed an integer $e \in\{0,2,3,4, \ldots\}$ and multicharge $\boldsymbol{\kappa}$. We now define the integral cyclotomic Hecke algebras $\mathscr{H}_{n}^{\Lambda}$ of type $G(\ell, 1, n)$, where $\ell=\sum_{i \in I}\left(\Lambda, \alpha_{i}\right)$ is the level of $\Lambda$.

Fix a field $K$. If $k \in \mathbb{Z}$ and $t \in K$ define the $t$-quantum integer

$$
[k]_{t}=\left\{\begin{array}{cl}
1+t+\cdots+t^{k-1}, & \text { if } k \geq 0, \\
-\left(t^{-1}+t^{-2}+\cdots+t^{k}\right), & \text { if } k<0 .
\end{array}\right.
$$

Now fix a non-element $\xi=\xi(e) \in K$ where $e$ is minimal such that $[e]_{\xi}=0$, if $e>1$, or $[k]_{\xi} \neq 0$ for all $k \in \mathbb{N}$, if $e=0$.

Following [16, we make the following definition.

2.7. Definition. Suppose that $K$ is a field containing a non-zero element $\xi=\xi(e)$ as above and let $\kappa \in \mathbb{Z}^{\ell}$ be a multicharge such that $\Lambda=\Lambda_{\kappa}$. The (integral) cyclotomic Hecke algebra $\mathscr{H}_{n}^{\Lambda}$ of type $G(\ell, 1, n)$ with Hecke parameter $\xi$ is the unital associative $K$-algebra with generators $L_{1}, \ldots, L_{n}, T_{1}, \ldots, T_{n-1}$ which are subject to the relations

$$
\begin{aligned}
& \prod_{l=1}^{\ell}\left(L_{1}-\left[\kappa_{l}\right]\right)=0, \quad\left(T_{r}+1\right)\left(T_{r}-\xi\right)=0, \\
& L_{r} L_{t}=L_{t} L_{r}, \quad T_{r} T_{s}=T_{s} T_{r} \quad \text { if }|r-s|>1, \\
& T_{s} T_{s+1} T_{s}=T_{s+1} T_{s} T_{s+1}, \quad T_{r} L_{t}=L_{t} T_{r}, \quad \text { if } t \neq r, r+1 \text {, } \\
& L_{r+1} T_{r}-T_{r} L_{r}=1+(\xi-1) L_{r+1},
\end{aligned}
$$

where $1 \leq r<n, 1 \leq s<n-1$ and $1 \leq t \leq n$.

The connection between the cyclotomic quiver Hecke algebras of type $\Gamma_{e}$ and the cyclotomic Hecke algebras of type $G(\ell, 1, n)$ is given by the following remarkable result of Brundan and Kleshchev.

2.8. Theorem (Brundan-Kleshchev's graded isomorphism theorem [4, Theorem 1.1]). Suppose $K$ is a field. Then $\mathscr{H}_{n}^{\Lambda} \cong \underline{\mathscr{R}}_{n}^{\Lambda}$.

In particular, over a field $K$, the cyclotomic quiver Hecke algebra $\mathscr{R}_{n}^{\Lambda}(K)=$ $\mathscr{R}_{n}^{\Lambda}(\mathbb{Z}) \otimes_{\mathbb{Z}} K$ has dimension $\ell^{n} n$ ! and, up to isomorphism, $\mathscr{H}_{n}^{\Lambda}$ depends only on $e$ and $\Lambda$. By [16], $\mathscr{R}_{n}^{\Lambda}(\mathbb{Z})$ is free of rank $\ell^{n} n$ ! if $e=0$ or if $e$ is a prime integer.

2.4. Partitions and tableaux. We now introduce the tableau combinatorics which describes the representation theory of $\mathscr{R}_{n}^{\Lambda}$.

A partition of $m$ is a sequence $\mu=\left(\mu_{1}, \mu_{2}, \ldots\right)$ of non-negative integers such that $m=|\mu|=\mu_{1}+\mu_{2}+\ldots$ and $\mu_{1} \geq \mu_{2} \geq \ldots$ An $\ell$-multipartition of $n$ is an ordered $\ell$-tuple of partitions $\boldsymbol{\mu}=\left(\mu^{(1)}|\ldots| \mu^{(\ell)}\right)$ such that $\left|\mu^{(1)}\right|+\cdots+\left|\mu^{(\ell)}\right|=$ $n$. The partition $\mu^{(l)}$ is the $l$ th component of $\boldsymbol{\mu}$. Let $\mathscr{P}_{n}^{\Lambda}$ be the set of all $\ell$ multipartitions of $n$. The set $\mathscr{P}_{n}^{\Lambda}$ depends only on $\ell$, and not on $\Lambda$; however, soon 
we will introduce the residues of a multipartition which will depend on $\Lambda$ or, more accurately, on $\boldsymbol{\kappa}$. When $\ell$ is clear from context we will talk of multipartitions.

We identify the multipartition $\boldsymbol{\mu} \in \mathscr{P}_{n}^{\Lambda}$ with its diagram

$$
\left\{(l, r, c) \in\{1, \ldots, \ell\} \times \mathbb{N}^{2} \mid 1 \leq c \leq \mu_{r}^{(l)}\right\} .
$$

The elements of (the diagram of) $\boldsymbol{\mu}$ are nodes. More generally, a node is any element of $\{1, \ldots, \ell\} \times \mathbb{N}^{2}$. We think of $\boldsymbol{\mu}$ as an $\ell$-tuple of arrays of boxes in the plane. For example, the diagram of $\left(3,1\left|1^{3}\right| 4,2\right)$ is

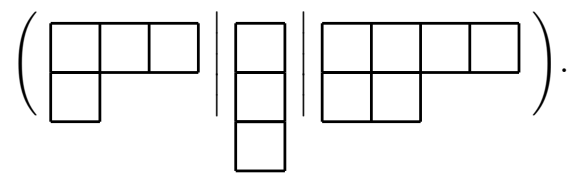

The length of a multipartition $\boldsymbol{\mu}$ is $\ell(\boldsymbol{\mu})=\#\left\{(l, r) \mid \mu_{r}^{(l)} \neq 0\right\}$. That is, $\ell(\boldsymbol{\mu})$ is the number of non-empty rows in the diagram of $\boldsymbol{\mu}$. A node $A \in \boldsymbol{\mu}$ is a removable node (of $\boldsymbol{\mu}$ ) if $\boldsymbol{\mu} \backslash\{A\}$ is (the diagram of) a multipartition. A node $B \notin \boldsymbol{\mu}$ is an addable node (for $\boldsymbol{\mu}$ ) if $\boldsymbol{\mu} \cup\{B\}$ is a multipartition. Let $\operatorname{Add}(\boldsymbol{\mu})$ and $\operatorname{Rem}(\boldsymbol{\mu})$ be the sets of addable and removable nodes of the multipartition $\boldsymbol{\mu}$.

Let $\boldsymbol{\mu}$ and $\boldsymbol{\nu}$ be multipartitions. Then $\boldsymbol{\mu}$ dominates $\boldsymbol{\nu}$, or $\boldsymbol{\mu} \unrhd \boldsymbol{\nu}$, if

$$
\sum_{l=1}^{m-1}\left|\mu^{(l)}\right|+\sum_{r=1}^{s} \mu_{r}^{(m)} \geq \sum_{l=1}^{m-1}\left|\nu^{(l)}\right|+\sum_{r=1}^{s} \nu_{r}^{(m)}
$$

for all $1 \leq m \leq \ell$ and $s \geq 1$. Therefore, $\boldsymbol{\mu} \unrhd \boldsymbol{\nu}$ if $\boldsymbol{\nu}$ is obtained from $\boldsymbol{\mu}$ by moving nodes down or to the right in the diagrams. We write $\boldsymbol{\mu} \triangleright \boldsymbol{\nu}$ if $\boldsymbol{\mu} \unrhd \boldsymbol{\nu}$ and $\boldsymbol{\mu} \neq \boldsymbol{\nu}$.

Let $\boldsymbol{\mu}$ be a multipartition. A $\boldsymbol{\mu}$-tableau $\mathfrak{t}$ is a bijection $\mathfrak{t}: \boldsymbol{\mu} \longrightarrow\{1,2, \ldots, n\}$. We think of a $\boldsymbol{\mu}$-tableau $\mathfrak{t}$ as a labelling of the diagram of $\boldsymbol{\mu}$ and we set Shape $(\mathfrak{t})=\boldsymbol{\mu}$. In this way we talk of the components, rows and columns of tableaux. In particular, if $\mathfrak{t}(l, r, c)=k$ then $k$ lies in row $(l, r)$ of $\mathfrak{t}$ and we write $\operatorname{row}_{\mathfrak{t}}(k)=(l, r)$. The rows of $\boldsymbol{\mu}$ (and the rows of $\mathfrak{t}$ ) are totally ordered lexicographically, from top left to bottom right, by defining

$$
(l, r)<\left(l^{\prime}, r^{\prime}\right) \quad \text { if } l<l^{\prime} \text { or if } l=l^{\prime} \text { and } r<r^{\prime} .
$$

The residue of a node $(l, r, c)$ is $\operatorname{res}(l, r, c)=\kappa_{l}-r+c(\bmod e)$, so that $\operatorname{res}(l, r, c) \in I$. An $i$-node is any node of residue $i$, for $i \in I$. Similarly, if $\mathfrak{t}$ is a tableau and $\mathfrak{t}(l, r, c)=k$ then the residue of $k$ in $\mathfrak{t}$ is $\operatorname{res}_{\mathfrak{t}}(k)=\operatorname{res}(l, r, c)$. The residue sequence of $\mathfrak{t}$ is

$$
\operatorname{res}(\mathfrak{t})=\left(\operatorname{res}_{\mathfrak{t}}(1), \operatorname{res}_{\mathfrak{t}}(2), \ldots, \operatorname{res}_{\mathfrak{t}}(n)\right) \in I^{n} .
$$

Note that the residues depend upon the choice of multicharge $\boldsymbol{\kappa}$.

A $\boldsymbol{\mu}$-tableau $\mathfrak{t}$ is row standard if its entries increase along the rows in each component. A $\boldsymbol{\mu}$-tableau $\boldsymbol{t}$ is standard if it is row standard and its entries increase down the columns in each component. Let $\operatorname{Std}(\boldsymbol{\mu})$ be the set of standard $\boldsymbol{\mu}$-tableaux.

If $\mathfrak{t}$ is a standard $\boldsymbol{\mu}$-tableau and $1 \leq k \leq n$ let $\mathfrak{t}_{\downarrow k}$ be the subtableau of $\mathfrak{t}$ which contains $1,2, \ldots, k$. Then $\operatorname{Shape}\left(\mathfrak{t}_{\downarrow k}\right)$ is a multipartition for $1 \leq k \leq n$. If $\mathfrak{s}, \mathfrak{t} \in \operatorname{Std}(\boldsymbol{\mu})$ then $\mathfrak{s}$ dominates $\mathfrak{t}$, and we write $\mathfrak{s} \unrhd \mathfrak{t}$, if

$$
\operatorname{Shape}\left(\mathfrak{s}_{\downarrow k}\right) \unrhd \operatorname{Shape}\left(\mathfrak{t}_{\downarrow k}\right), \quad \text { for } 1 \leq k \leq n .
$$

This defines a partial order on the set of row standard $\boldsymbol{\mu}$-tableaux. 
Let $\mathfrak{t}^{\boldsymbol{\mu}}$ be the unique row standard $\boldsymbol{\mu}$-tableau such that $\mathfrak{t}^{\boldsymbol{\mu}} \unrhd \mathfrak{t}$, for all $\mathfrak{t} \in \operatorname{Std}(\boldsymbol{\mu})$. Then $\mathfrak{t}^{\boldsymbol{\mu}}$ has the numbers $1,2, \ldots, n$ entered in order from left to right along its rows. Set $\mathbf{i}^{\mu}=\operatorname{res}\left(\mathfrak{t}^{\boldsymbol{\mu}}\right)$. For any $\boldsymbol{\mu}$-tableau $\mathfrak{t}$ let $d(\mathfrak{t}) \in \mathfrak{S}_{n}$ be the unique permutation such that $\mathfrak{t}=\mathfrak{t}^{\mu} d(\mathfrak{t})$. It is a well-known result, commonly attributed to Ehresmann and James, that $\mathfrak{s} \unrhd \mathfrak{t}$ if and only if $d(\mathfrak{s}) \leq d(\mathfrak{t})$, where $\leq$ is the Bruhat order on $\mathfrak{S}_{n}$; see, for example, [24, Theorem 3.8].

If $\boldsymbol{\mu} \in \mathscr{P}_{n}^{\Lambda}$ and $A$ is a removable $i$-node of $\boldsymbol{\mu}$, let

$$
\begin{aligned}
d_{A}(\boldsymbol{\mu})=\#\{\text { addable } i \text {-nodes of } \boldsymbol{\mu} \text { strictly below } A\} \\
\qquad \text { - \#\{removable } i \text {-nodes of } \boldsymbol{\mu} \text { strictly below } A\} .
\end{aligned}
$$

For $\mathfrak{t} \in \operatorname{Std}(\boldsymbol{\mu})$, we define the degree of $\mathfrak{t}$ recursively as follows. If $n=0$ then $\operatorname{deg} \mathfrak{t}=0$. Otherwise let $A=\mathfrak{t}^{-1}(n)$ be the node occupied by $n$ in $\mathfrak{t}$ and set

$$
\operatorname{deg} \mathfrak{t}=\operatorname{deg} \mathfrak{t} \backslash\{A\}+d_{A}(\boldsymbol{\mu}) .
$$

This degree function describes the grading on the Specht module $S^{\mu}$.

2.5. Graded Specht modules. The graded Specht modules were first defined over a field by Brundan, Kleshchev and Wang [6]. In this paper we follow the approach of [20] which defines the Specht modules by generators and relations over an arbitrary ring. The main advantage of using [20] is that the graded Specht modules are defined over $\mathbb{Z}$ and the action of $\mathscr{R}_{n}^{\Lambda}(\mathbb{Z})$ on these modules is explicitly described in terms of the KLR generators. In contrast, using the definitions in [6], it is only possible to compute inside the graded Specht modules by repeatedly applying Theorem 2.8

We remark that [15] gives a third construction of the graded Specht modules using cellular algebra techniques. This approach equips the graded Specht modules with a homogeneous bilinear form. We do not need this result here.

Fix a multipartition $\boldsymbol{\mu}$ and a node $A=(l, r, c) \in \boldsymbol{\mu}$. If $(l, r+1, c) \in \boldsymbol{\mu}$ then $A$ is a (row) Garnir node of $\boldsymbol{\mu}$. Suppose that $e \neq 0$. Then the $(e, A)$-Garnir belt is the set of nodes

$$
\begin{array}{r}
\mathbf{B}_{A}^{(e)}=\left\{(l, r, k) \in \boldsymbol{\mu} \mid k \geq c \text { and } e\left\lceil\frac{k-c+1}{e}\right\rceil \leq \mu_{r}^{(l)}-c+1\right\} \\
\cup\left\{(l, r+1, k) \in \boldsymbol{\mu} \mid k \leq c \text { and } c \geq e\left\lceil\frac{c-k+1}{e}\right\rceil\right\} .
\end{array}
$$

Let $b_{A}=\# \mathbf{B}_{A}^{(e)} / e$ and write $b_{A}=a_{A}+c_{A}$ where $e a_{A}$ is the number of nodes in $\mathbf{B}_{A}^{(e)}$ in row $(l, r)$. Let $\mathscr{D}_{A}$ be the set of minimal length right coset representatives of $\mathfrak{S}_{a_{A}} \times \mathfrak{S}_{c_{A}}$ in $\mathfrak{S}_{b_{A}}$; see, for example, [24, Proposition 3.3]. If $e=0$, set $\mathbf{B}_{A}^{(e)}=\emptyset$, $b_{A}=0=a_{A}=c_{A}$ and $\mathscr{D}_{A}=1$.

Suppose $A$ is a Garnir node of $\boldsymbol{\mu}$. Let $\mathrm{T}_{A}^{(e)}$ be the $\boldsymbol{\mu}$-tableau which agrees with $\mathfrak{t}^{\boldsymbol{\mu}}$ for all numbers $k<\mathfrak{t}^{\mu}(A)=\mathfrak{t}^{\mu}(l, r, c)$ and $k>\mathfrak{t}^{\mu}(l, r+1, c)$ and where the remaining entries in rows $(l, r)$ and $(l, r+1)$ are filled in increasing order from left to right first along the nodes in row $(l, r+1)$ which are in the first $c$ columns but not in $\mathbf{B}_{A}^{(e)}$, then along the nodes in row $(l, r)$ of $\mathbf{B}_{A}^{(e)}$ followed by the nodes in row $(l, r+1)$ of $\mathbf{B}_{A}^{(e)}$, and then along the remaining nodes in row $(l, r)$.

2.11. Example. Suppose that $e=2, \boldsymbol{\mu}=(3,1|7,5| 2,1)$ and $A=(1,3,2)$. Then

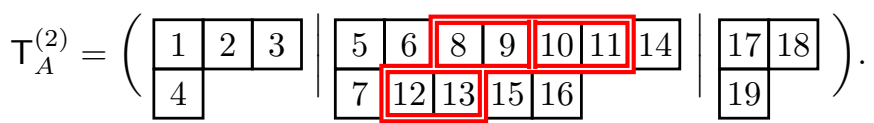


The double lines in $\mathrm{T}_{A}^{(2)}$ show the $(2, A)$-Garnir belt of $\boldsymbol{\mu}$ and they show how it decomposes into a disjoint union of "e-bricks". In general, if $e \neq 0$ then $b_{A}$ is the number of $e$-bricks in the Garnir belt and $a_{A}$ is the number of $e$-bricks in its first row. In this case, $b_{A}=3$ and $a_{A}=2$. Therefore, $\mathscr{D}_{A}=\left\{1, s_{2}, s_{2} s_{1}\right\}$.

Let $k_{A}=\mathrm{T}_{A}^{(e)}(A)$ be the number occupying $A$ in $\mathrm{T}_{A}^{(e)}$. For $1 \leq r<b_{A}$ define

$$
w_{r}^{A}=\prod_{a=k_{A}+e(r-1)}^{k_{A}+r e-1}(a, a+e) .
$$

Although it does not affect the last definition, because all of the factors commute, in this paper we use the convention that all products are read from left to right. The set $\left\{w_{r}^{A} \mid 1 \leq r<b_{A}\right\}$ generates a subgroup of $\mathfrak{S}_{n}$ isomorphic to $\mathfrak{S}_{b_{A}}$, where the isomorphism is determined by the map $w_{r}^{A} \mapsto s_{r}$, for $1 \leq r<b_{A}$. Set $\mathbf{i}_{A}=\operatorname{res}\left(\mathbf{T}_{A}^{(e)}\right)$. If $d \in \mathscr{D}_{A}$ choose a reduced expression $d=s_{r_{1}} \ldots s_{r_{k}}$ for $d$ and define

$$
\tau_{d}^{A}=e\left(\mathbf{i}_{A}\right)\left(\psi_{w_{r_{1}}^{A}}+1\right) \ldots\left(\psi_{w_{r_{k}}^{A}}+1\right) \in \mathscr{R}_{n}^{\Lambda} .
$$

Then $\tau_{d}^{A}$ is independent of the choice of reduced expression for $d$ and independent of the choice of reduced expression for each $\psi_{w_{r_{i}}^{A}}$ by [20, Theorem 5.11]. We can now define the Specht modules of $\mathscr{R}_{n}^{\Lambda}$.

2.12. Definition ([20, Definition 5.9]). Suppose that $\boldsymbol{\mu} \in \mathscr{P}_{n}^{\Lambda}$. The Specht module $S^{\mu}$ of $\mathscr{R}_{n}^{\Lambda}$ is the $\mathscr{R}_{n}^{\Lambda}$-module generated by the homogeneous element $v_{t^{\mu}}$ of degree $\operatorname{deg} \mathfrak{t}^{\mu}$ subject to the relations:

a) $v_{\mathfrak{t}} \mu e(\mathbf{i})=\delta_{\mathbf{i i}^{\mu}} v_{\mathfrak{t}}^{\mu}$.

b) $v_{t^{\mu}} y_{s}=0$, for $1 \leq s \leq n$.

c) $v_{\mathfrak{t}} \mu \psi_{r}=0$ whenever $r$ and $r+1$ are in the same row of $\mathfrak{t}^{\mu}$, for $1 \leq r<n$.

d) $\sum_{d \in \mathscr{D}_{A}} v_{\mathfrak{t}^{\mu}} \psi_{\mathrm{T}_{A}^{(e)}} \tau_{d}^{A}=0$, for all Garnir nodes $A \in \boldsymbol{\mu}$.

The relations in part (d) are the homogeneous Garnir relations. By [20, Theorem 6.23], if $K$ is a field then the graded Specht module $S^{\boldsymbol{\mu}} \otimes_{\mathbb{Z}} K$ is isomorphic, as a graded $\mathscr{R}_{n}^{\Lambda}$-module, to the graded Specht module studied previously by Brundan, Kleshchev and Wang [6] and by $\mathrm{Hu}$ and Mathas [15.

For any $\boldsymbol{\mu}$-tableau $\mathfrak{t}$ define $v_{\mathfrak{t}}=v_{\mathfrak{t}} \mu \psi_{d(\mathfrak{t})} \in S^{\boldsymbol{\mu}}$. These elements depend on the choice of reduced expression for the permutation $d(\mathfrak{t})$. Nonetheless, by fixing arbitrary choices of these reduced expressions we have the following:

2.13. Theorem ([6, Theorem 4.2], [20, Theorem 6.21]). Suppose that $\boldsymbol{\mu} \in \mathscr{P}_{n}^{\Lambda}$. Then $\left\{v_{\mathfrak{t}} \mid \mathfrak{t} \in \operatorname{Std}(\boldsymbol{\mu})\right\}$ is a homogeneous basis of $S^{\boldsymbol{\mu}}$ with $\operatorname{deg} v_{\mathfrak{t}}=\operatorname{deg} \mathfrak{t}$, for all $\mathfrak{t} \in \operatorname{Std}(\boldsymbol{\mu})$.

For future use we now state three properties of the homogeneous basis of $S^{\mu}$ which go back to results of Brundan, Kleshchev and Wang 6]. In view of 20, Theorem 6.23] these results are valid over $\mathbb{Z}$. First observe that Definition 2.12 (a) and the relation $\psi_{r} e(\mathbf{i})=e\left(s_{r} \mathbf{i}\right) \psi_{r}$ imply that if $\mathfrak{t}$ is any $\boldsymbol{\mu}$-tableau then

$$
v_{\mathfrak{t}} e(\mathbf{i})=\delta_{\mathbf{i}, \operatorname{res}(\mathfrak{t})} v_{\mathfrak{t}},
$$

for all $\mathbf{i} \in I^{n}$. 
2.15. Lemma ([6, Corollary 4.6 \& Proposition 4.7]). Let $s_{i_{1}} s_{i_{2}} \ldots s_{i_{l}}$ be a reduced expression for a permutation $w \in \mathfrak{S}_{n}$ such that $\mathfrak{t}=\mathfrak{t}^{\boldsymbol{\mu}} w$ is standard. Then

$$
v_{\mathfrak{t} \mu} \psi_{i_{1}} \psi_{i_{2}} \ldots \psi_{i_{l}}=\sum_{\substack{\mathfrak{s} \in \operatorname{Std}(\boldsymbol{\mu}), \mathfrak{s} \unrhd \mathfrak{t}}} a_{\mathfrak{s}} v_{\mathfrak{s}}
$$

for some $a_{\mathfrak{s}} \in \mathbb{Z}$. Furthermore $a_{\mathfrak{s}} \neq 0$ only if $\operatorname{res}(\mathfrak{s})=\operatorname{res}(\mathfrak{t})$.

2.16. Lemma ([6, Lemma 4.8]). Suppose that $\mathfrak{t} \in \operatorname{Std}(\boldsymbol{\nu})$ and $1 \leq r \leq n$. Then

$$
v_{\mathfrak{t}} y_{r}=\sum_{\substack{\mathfrak{s} \in \operatorname{Std}(\boldsymbol{\mu}), \mathfrak{s} \triangleright \mathfrak{t}}} a_{\mathfrak{s}} v_{\mathfrak{s}}
$$

for some $a_{\mathfrak{s}} \in \mathbb{Z}$. Furthermore if $a_{\mathfrak{s}} \neq 0$ then $\operatorname{res}(\mathfrak{s})=\operatorname{res}(\mathfrak{t})$.

2.6. Braid diagrams. This section introduces the diagram combinatorics of Khovanov and Lauda [18, §2], adapting it to our situation. In principle, all of the arguments in this paper could be given algebraically, however, they become much more transparent, and shorter, when we use these diagrams.

An $n$-braid diagram is an $I$-labelled graph on $\left\{1^{\prime}, \ldots, n^{\prime}, 1, \ldots, n\right\}$ such that each vertex in $\left\{1^{\prime}, \ldots, n^{\prime}\right\}$ is connected to a unique vertex in $\{1, \ldots, n\}$ and each edge is labelled by an element of $I$ and is decorated by a finite, non-negative, number of dots.

As we explain below, the braid diagrams in this paper represent an element of a Specht module $S^{\mu}$, for some multipartition $\boldsymbol{\mu} \in \mathscr{P}_{n}^{\Lambda}$. All of the relations in $\mathscr{R}_{n}^{\Lambda}$ and in $S^{\boldsymbol{\mu}}$ have diagrammatic counterparts which describe how to rewrite a given diagram as a linear combination of other diagrams. Considered as a map from $\left\{1^{\prime}, \ldots, n^{\prime}\right\}$ to $\{1, \ldots, n\}$, each braid diagram $B$ determines a unique permutation $\pi_{B} \in \mathfrak{S}_{n}$. We emphasize that the element of $S^{\boldsymbol{\mu}}$ corresponding to $B$ is, in general, not determined by $\pi_{B}$ and is dependent on the crossings and the dots in $B$.

We represent braid diagrams as graphs in the plane and we do not distinguish between under and over crossings. Braid diagrams are drawn with the vertices labelled by $\left\{1^{\prime}, \ldots, n^{\prime}\right\}$ at the top of the diagram and the vertices labelled by $\{1, \ldots, n\}$ at the bottom, with both sets of vertices ordered from left to right in the obvious way. For $1 \leq m \leq n$ the (unique) edge with vertex $m$ is the $m$-string of residue $i$, where $i \in I$ is the corresponding edge label. We omit the labels for the vertices $\left\{1^{\prime}, \ldots, n^{\prime}\right\}$ and put the edge labels $\mathbf{i}=\left(i_{1}, \ldots, i_{n}\right) \in I^{n}$ at the top of each string. To improve readability, we colour the strings of the diagrams, however, these colours have no mathematical meaning (and may not be distinguishable in black and white!).

Writing $\mathbf{i}^{\mu}=\left(i_{1}, \ldots, i_{n}\right)$, the braid diagram representing $v_{t^{\mu}} \in S^{\mu}$ is:

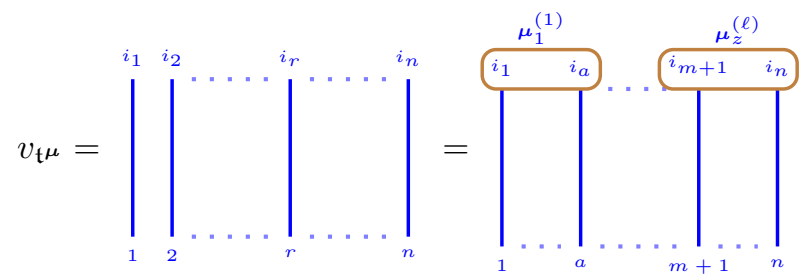

As in the right-hand diagram (where $a=\mu_{1}^{(1)}, m=n-\mu_{z}^{(\ell)}<n$ and $\mu_{z+1}^{(\ell)}=0$ ), we will sometimes (but not always) indicate the rows of $\boldsymbol{\mu}$ by drawing a circle around the corresponding residues at the top of the diagram. 
For $1 \leq r<n, \psi_{r}$ acts on a braid diagram by crossing the $r$-string and the $(r+1)$-string at the bottom of the diagram. If $1 \leq s \leq n$ then $y_{s}$ acts by putting a dot at the bottom of the $s$-string. If $\mathbf{j} \in I^{n}$ then $e(\mathbf{j})$ acts as the identity on a diagram $B$ if $\mathbf{j}=\pi_{B}^{-1} \cdot \mathbf{i}$, and otherwise it acts as zero.

All of the relations in Definitions 2.2 and 2.12 have corresponding diagrammatic versions. For example, the relations in parts (b) and (c) of Definition 2.12 become:

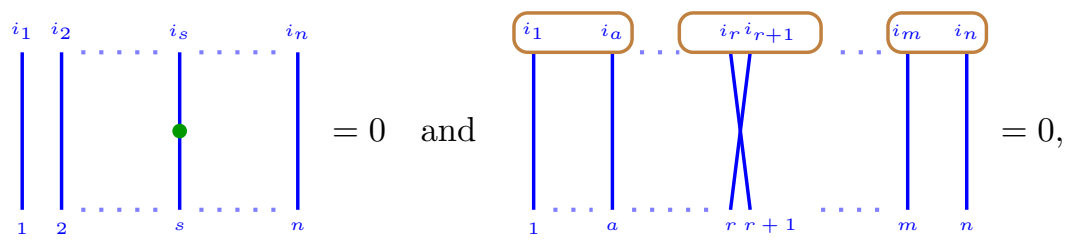

respectively. Similarly when $e \neq 2$, relations (2.4) and (2.6) yield the diagrams:
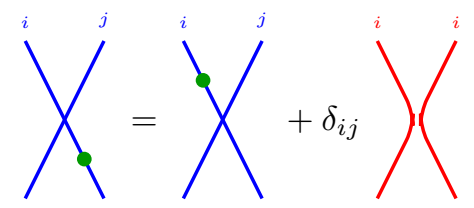

and

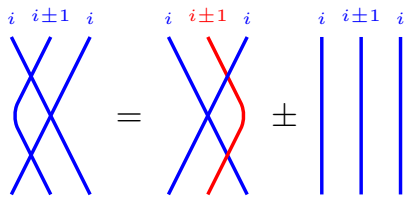

both of which should be viewed as "local" relations for strings inside a braid diagram. The homogeneous Garnir relations of Definition 2.12 (d) are harder to represent diagrammatically, however, in the special case when $A=(l, r, c)$ and either $e=0$ or $\delta=\boldsymbol{\mu}_{r}^{(l)}-c+1<e$ this relation simplifies to $v_{t^{\mu}} \psi_{\mathrm{T}_{A}^{(e)}}=0$, or

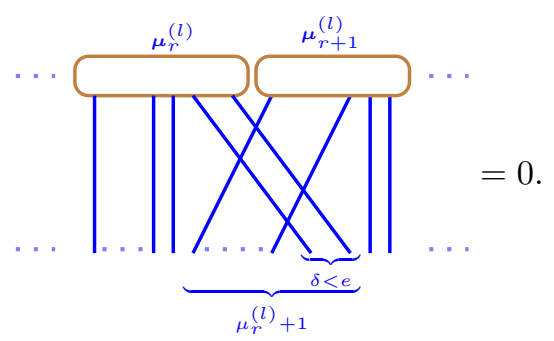

We will use these diagrams extensively in this paper. The reader who has not used these diagrams before may find, initially, that they are cumbersome to work with. The reader should persevere, however, because these diagrams allow us to replace long calculations with the KLR relations with one or two diagrams which make the arguments more transparent. As these diagrams take some getting use to we give a toy example of how they are used which also illustrates why they are useful.

2.20. Example. Take $e=2$, let $\mu$ be the partition $(5,4)$ and consider $v_{\mathfrak{t}}=$ $v_{\mathfrak{t}^{\mu}} \psi_{5} \psi_{6} \psi_{7} \psi_{8} y_{9}$, where $\mathfrak{t}=$\begin{tabular}{|l|l|l|l}
\hline 1 & 2 & 3 & 4 \\
\hline 5 & 6 & 7 & 8 \\
\hline
\end{tabular} . Let $i=\operatorname{res}_{\mathfrak{t}^{\mu}}(5)=0$. This product corresponds to:

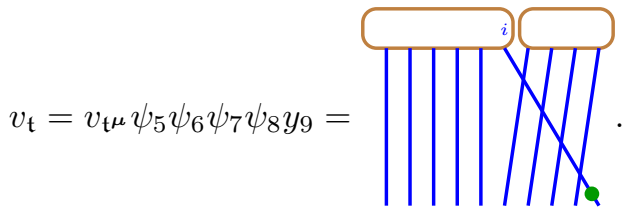


The dot at the bottom of the diagram represents $y_{9}$ because it is on the 9 -string, which is the ninth string reading from left to right at the bottom of the diagram. The 9 -string has residue 0 because its top vertex is $5^{\prime}$, the fifth string reading left to right at the top of the diagram, and $\operatorname{res}_{t} \mu(m)=i$. The 9-string crosses the four $k$-strings, for $k=5,6,7,8$. Reading these crossings in order, from top to bottom, corresponds to multiplying by $\psi_{5}, \ldots, \psi_{8}$, respectively.

Now it is, of course, easy to compute this example using the relations. Diagrammatically, it is even easier because (2.18) says that we slide the dot all the way up to the top of the 9-string, where it comes zero, except that whenever we cross another string of residue $i$ then we must add the a new diagram created by removing the dot and cutting the ${ }^{i} \mathbf{X}^{i}$-crossing. The 8 -string and the 6 -string have residue $i$, so

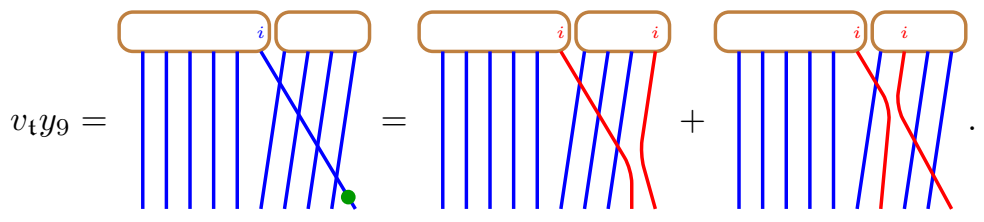

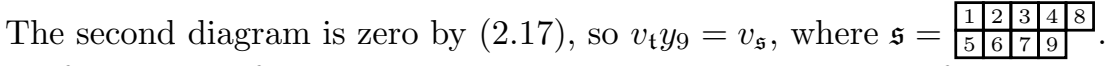

If the reader if not yet convinced that these diagrams simplify these calculations, then exactly the same argument shows that if $e \in\{0,2,3, \ldots\}$ and $\mu=(m, n-m)$, where $m \leq n \leq 2 m$, then

$$
v_{\mathfrak{t} \mu} \psi_{m} \psi_{m+1} \ldots \psi_{n-1} y_{n}= \begin{cases}v_{\mathfrak{s}}, & \operatorname{res}_{\mathfrak{t} \mu}(m)=\operatorname{res}_{\mathfrak{t} \mu}(n), \\ 0, & \text { otherwise, }\end{cases}
$$

where $\mathfrak{s}=\mathfrak{t}^{\mu} s_{m} \ldots s_{n-2}$.

2.7. Restricting Specht modules. We now describe the framework that we use to prove our Main Theorem. These ideas are a natural extension of work of Ellers and Murray [13.

Let $\boldsymbol{\nu}$ be a multipartition of $n+1$. By [6, Theorem 4.11] and [20, Theorem 6.23], the restriction $\operatorname{Res} S^{\nu}$ of $S^{\nu}$ to $\mathscr{R}_{n}^{\Lambda}$ has a graded Specht module filtration

$$
S^{\nu}=S_{0}^{\nu} \supset S_{1}^{\nu} \supset \cdots \supset S_{z+1}^{\nu}=0
$$

where $S_{k}^{\boldsymbol{\nu}} / S_{k+1}^{\boldsymbol{\nu}} \cong S^{\boldsymbol{\nu} \backslash\left\{A_{k+1}\right\}}\left\langle\operatorname{deg} A_{k+1}\right\rangle$, for $0 \leq k \leq z$, and $A_{1}<\cdots<A_{z+1}$ are the removable nodes of $\boldsymbol{\nu}$, where the nodes $A_{1}, \ldots, A_{z+1}$ are ordered lexicographically so that $\operatorname{row}\left(A_{1}\right)<\cdots<\operatorname{row}\left(A_{z+1}\right)$ according to (2.9), In particular, $S_{k}^{\nu}$ is the $\mathscr{R}_{n}^{\Lambda}$-submodule of $S^{\boldsymbol{\nu}}$ with basis $\left\{v_{\mathfrak{t}} \mid \mathfrak{t} \in \operatorname{Std}(\boldsymbol{\nu})\right.$ and $\left.\operatorname{Shape}\left(\mathfrak{t}_{\downarrow n}\right) \unrhd \boldsymbol{\nu} \backslash\left\{A_{k+1}\right\}\right\}$.

More generally, suppose that $\nu \in \mathscr{P}_{n+\gamma}^{\Lambda}$ where $\gamma>0$. The next result then follows by considering the restriction of $S^{\nu}$ to $\mathscr{R}_{n}^{\Lambda}$ and repeatedly applying (2.21).

2.22. Lemma. Suppose that $\mathfrak{t} \in \operatorname{Std}(\boldsymbol{\nu})$ and that $h \in \mathscr{R}_{n}^{\Lambda}$. Then

$$
v_{\mathfrak{t}} h=\sum_{\mathfrak{s} \in \operatorname{Std}(\boldsymbol{\nu})} a_{\mathfrak{s}} v_{\mathfrak{s}}
$$

where $a_{\mathfrak{s}} \neq 0$ only if $\operatorname{row}_{\mathfrak{s}}(n+g) \geq \operatorname{row}_{\mathfrak{t}}(n+g)$ for $1 \leq g \leq \gamma$. 
If $\mathfrak{t} \in \operatorname{Std}(\boldsymbol{\nu})$, let $\operatorname{Std}_{\mathfrak{t}}^{\gamma}(\boldsymbol{\nu})=\left\{\mathfrak{s} \in \operatorname{Std}(\boldsymbol{\nu}) \mid \mathfrak{s}^{-1}(n+g)=\mathfrak{t}^{-1}(n+g)\right.$ for $\left.1 \leq g \leq \gamma\right\}$. Now suppose that $\boldsymbol{\sigma} \in \mathscr{P}_{n}^{\Lambda}$ and that $\boldsymbol{\sigma} \subset \boldsymbol{\nu}$. Let

$$
\begin{aligned}
& \operatorname{Std}_{\boldsymbol{\sigma}}^{\vee}(\boldsymbol{\nu})=\left\{\mathfrak{s} \in \operatorname{Std}(\boldsymbol{\nu}) \mid \operatorname{Shape}\left(\mathfrak{s}_{\downarrow n}\right) \triangleright \boldsymbol{\sigma}\right\}, \\
& \operatorname{Std}_{\boldsymbol{\sigma}}^{\wedge}(\boldsymbol{\nu})=\left\{\mathfrak{s} \in \operatorname{Std}(\boldsymbol{\nu}) \mid \boldsymbol{\sigma} \unrhd \operatorname{Shape}\left(\mathfrak{s}_{\downarrow n}\right)\right\} .
\end{aligned}
$$

Then $\operatorname{Std}_{\boldsymbol{\sigma}}^{\vee}(\boldsymbol{\nu}) \subseteq \operatorname{Std}_{\boldsymbol{\sigma}}^{\wedge}(\boldsymbol{\nu})$ where this inclusion is usually strict if $\gamma>1$. If $\mathfrak{t}_{\boldsymbol{\sigma}} \in$ $\operatorname{Std}(\boldsymbol{\nu})$ and $\left(\mathfrak{t}_{\boldsymbol{\sigma}}\right)_{\downarrow n}=\mathfrak{t}^{\boldsymbol{\sigma}}$ then define

$$
\begin{array}{ll}
\check{S}_{\mathfrak{t}_{\boldsymbol{\sigma}}}^{\nu}=\left\langle v_{\mathfrak{s}} \mid \mathfrak{s} \in \operatorname{Std}_{\mathfrak{t}_{\boldsymbol{\sigma}}}^{\gamma}(\boldsymbol{\nu}) \cup \operatorname{Std}_{\boldsymbol{\sigma}}^{\vee}(\boldsymbol{\nu})\right\rangle_{\mathbb{Z}}, & \check{S}_{\boldsymbol{\sigma}}^{\nu}=\left\langle v_{\mathfrak{s}} \mid \mathfrak{s} \in \operatorname{Std}_{\boldsymbol{\sigma}}^{\vee}(\boldsymbol{\nu})\right\rangle_{\mathbb{Z}}, \\
\hat{S}_{\mathfrak{t}_{\boldsymbol{\sigma}}}^{\nu}=\left\langle v_{\mathfrak{s}} \mid \mathfrak{s} \in \operatorname{Std}_{\mathfrak{t}_{\boldsymbol{\sigma}}}^{\gamma}(\boldsymbol{\nu}) \cup \operatorname{Std}_{\boldsymbol{\sigma}}^{\wedge}(\boldsymbol{\nu})\right\rangle_{\mathbb{Z}}, & \hat{S}_{\boldsymbol{\sigma}}^{\nu}=\left\langle v_{\mathfrak{s}} \mid \mathfrak{s} \in \operatorname{Std}_{\boldsymbol{\sigma}}^{\wedge}(\boldsymbol{\nu})\right\rangle_{\mathbb{Z}} .
\end{array}
$$

Fix multipartitions $\boldsymbol{\lambda}$ and $\boldsymbol{\mu}$ of $n$ such that $\boldsymbol{\mu} \triangleright \boldsymbol{\lambda}$ and $\boldsymbol{\lambda}, \boldsymbol{\mu} \subset \boldsymbol{\nu}$ and fix standard tableaux $\mathfrak{t}_{\boldsymbol{\lambda}}, \mathfrak{t}_{\boldsymbol{\mu}} \in \operatorname{Std}(\boldsymbol{\nu})$ such that $\left(\mathfrak{t}_{\boldsymbol{\lambda}}\right)_{\downarrow n}=\mathfrak{t}^{\boldsymbol{\lambda}},\left(\mathfrak{t}_{\boldsymbol{\mu}}\right)_{\downarrow n}=\mathfrak{t}^{\boldsymbol{\mu}}$ and $\mathfrak{t}_{\boldsymbol{\mu}} \triangleright \mathfrak{t}_{\boldsymbol{\lambda}}$. It follows from Lemma 2.22 that $\check{S}_{\mathfrak{t}_{\boldsymbol{\lambda}}}^{\nu}, \check{S}_{\boldsymbol{\lambda}}^{\nu}, \hat{S}_{\mathfrak{t}_{\mu}}^{\nu}$ and $\hat{S}_{\boldsymbol{\mu}}^{\nu}$ are all $\mathscr{R}_{n}^{\Lambda}$-submodules of $S^{\nu}$ and, moreover, that $S^{\nu}$ has an $\mathscr{R}_{n}^{\Lambda}$-module filtration

$$
S^{\nu} \supseteq \check{S}_{\mathfrak{t}_{\lambda}}^{\nu} \supset \check{S}_{\lambda}^{\nu} \supseteq \hat{S}_{\mathfrak{t}_{\mu}}^{\nu} \supset \hat{S}_{\mu}^{\nu} \supset 0 .
$$

Furthermore, $S^{\boldsymbol{\lambda}}\left\langle\operatorname{deg} \mathfrak{t}_{\boldsymbol{\lambda}}-\operatorname{deg} \mathfrak{t}^{\boldsymbol{\lambda}}\right\rangle \cong \check{S}_{\mathfrak{t}_{\boldsymbol{\lambda}}}^{\nu} / \check{S}_{\boldsymbol{\lambda}}^{\nu}$ and $S^{\boldsymbol{\mu}}\left\langle\operatorname{deg} \mathfrak{t}_{\boldsymbol{\mu}}-\operatorname{deg} \mathfrak{t}^{\boldsymbol{\mu}}\right\rangle \cong \hat{S}_{\mathfrak{t}_{\mu}}^{\nu} / \hat{S}_{\boldsymbol{\mu}}^{\nu}$ as graded $\mathscr{R}_{n}^{\Lambda}$-modules where the isomorphisms are given by $v_{\mathfrak{s}}+\check{S}_{\boldsymbol{\lambda}}^{\nu} \mapsto v_{\mathfrak{s}_{\downarrow n}}$ and $v_{\mathfrak{t}}+\hat{S}_{\boldsymbol{\mu}}^{\nu} \mapsto v_{\mathfrak{t}_{\downarrow n}}$, for $\mathfrak{s} \in \operatorname{Std}_{\mathfrak{t}_{\boldsymbol{\lambda}}}^{\gamma}(\boldsymbol{\nu})$ and $\mathfrak{t} \in \operatorname{Std}_{\mathfrak{t}_{\boldsymbol{\mu}}}(\boldsymbol{\nu})$, respectively. In particular, as $\mathscr{R}_{n}^{\Lambda}$-modules, $\check{S}_{\mathfrak{t}_{\boldsymbol{\lambda}}}^{\nu} / \check{S}_{\boldsymbol{\lambda}}^{\nu}$ is generated by $v_{\mathfrak{t}_{\boldsymbol{\lambda}}}+\check{S}_{\boldsymbol{\lambda}}^{\nu}$ and, similarly, $\hat{S}_{\mathfrak{t}_{\mu}}^{\nu} / \hat{S}_{\boldsymbol{\mu}}^{\nu}$ is generated by $v_{\mathfrak{t}_{\mu}}+\hat{S}_{\mu}^{\nu}$.

The following result is a graded analogue of [23, Corollary 2.3].

2.24. Lemma. For $\boldsymbol{\nu} \in \mathscr{P}_{n+\gamma}^{\Lambda}$ and $\boldsymbol{\lambda}, \boldsymbol{\mu} \in \mathscr{P}_{n}^{\Lambda}$ with $\boldsymbol{\lambda}, \boldsymbol{\mu} \subset \boldsymbol{\nu}$, let $\mathfrak{t}_{\boldsymbol{\lambda}}, \mathfrak{t}_{\boldsymbol{\mu}} \in \operatorname{Std}(\boldsymbol{\nu})$ be standard tableaux such that $\left(\mathfrak{t}_{\boldsymbol{\lambda}}\right)_{\downarrow n}=\mathfrak{t}^{\boldsymbol{\lambda}},\left(\mathfrak{t}_{\boldsymbol{\mu}}\right)_{\downarrow n}=\mathfrak{t}^{\boldsymbol{\mu}}$ and $\mathfrak{t}_{\boldsymbol{\mu}} \triangleright \mathfrak{t}_{\boldsymbol{\lambda}}$. Suppose that there exists a homogeneous element $L \in \mathscr{R}_{n+\gamma}^{\Lambda}$ such that $L$ commutes with $\mathscr{R}_{n}^{\Lambda}$ and

$$
\check{S}_{\mathfrak{t}_{\lambda}}^{\nu} L \subseteq \hat{S}_{\mathfrak{t}_{\mu}}^{\nu}, \quad \check{S}_{\lambda}^{\nu} L \subseteq \hat{S}_{\boldsymbol{\mu}}^{\nu} \quad \text { and } \quad v_{\mathfrak{t}_{\lambda}} L \notin \hat{S}_{\boldsymbol{\mu}}^{\nu} .
$$

Then $\operatorname{Hom}_{\mathscr{R}_{n}^{\Lambda}}\left(S^{\boldsymbol{\lambda}}\langle d\rangle, S^{\boldsymbol{\mu}}\right) \neq 0$, where $d=\operatorname{deg} \mathfrak{t}_{\boldsymbol{\lambda}}-\operatorname{deg} \mathfrak{t}^{\boldsymbol{\lambda}}-\operatorname{deg} \mathfrak{t}_{\boldsymbol{\mu}}+\operatorname{deg} \mathfrak{t}^{\boldsymbol{\mu}}+\operatorname{deg} L$.

Proof. Since $L$ commutes with $\mathscr{R}_{n}^{\Lambda}$, our assumptions ensure that there is a welldefined, non-zero, degree preserving $\mathscr{R}_{n}^{\Lambda}$-module homomorphism such that

$$
\theta_{L}: \check{S}_{\mathfrak{t}_{\boldsymbol{\lambda}}}^{\nu} / \check{S}_{\boldsymbol{\lambda}}^{\nu}\langle\operatorname{deg} L\rangle \longrightarrow \hat{S}_{\mathfrak{t}_{\mu}}^{\nu} / \hat{S}_{\boldsymbol{\mu}}^{\nu} ; \quad x+\check{S}_{\boldsymbol{\lambda}}^{\nu} \mapsto x L+\hat{S}_{\boldsymbol{\mu}}^{\nu},
$$

for $x \in \check{S}_{\mathfrak{t}_{\boldsymbol{\lambda}}}^{\nu}$. By construction, $\check{S}_{\mathfrak{t}_{\boldsymbol{\lambda}}}^{\nu} / \check{S}_{\boldsymbol{\lambda}}^{\nu} \cong S^{\boldsymbol{\lambda}}\left\langle\operatorname{deg} \mathfrak{t}_{\boldsymbol{\lambda}}-\operatorname{deg} \mathfrak{t}^{\boldsymbol{\lambda}}\right\rangle$ and $\hat{S}_{\mathfrak{t}^{\mu}}^{\nu} / \hat{S}_{\boldsymbol{\mu}}^{\nu} \cong$ $S^{\boldsymbol{\mu}}\left\langle\operatorname{deg} \mathfrak{t}_{\boldsymbol{\mu}}-\operatorname{deg} \mathfrak{t}^{\boldsymbol{\mu}}\right\rangle$ as $\mathscr{R}_{n}^{\Lambda}$-modules, so the result follows.

\section{Cyclotomic Carter-Payne pairs}

In this section we prove our Main Theorem by using the framework of Lemma 2.24 to explicitly construct a non-zero homomorphism between two Specht modules $S^{\boldsymbol{\lambda}}$ and $S^{\boldsymbol{\mu}}$ when $(\boldsymbol{\lambda}, \boldsymbol{\mu})$ is a cyclotomic Carter-Payne pair. The first section treats the simplest case, when $\boldsymbol{\lambda}$ and $\boldsymbol{\mu}$ differ by one node. This case is easy to understand and it illustrates some of the main ideas of the general case. Doing the one node case first also has the advantage that it allows us to assume that $e \neq 2$ in later sections. Once the one node case is settled we introduce the machinery that we need to tackle the general case and then prove our Main Theorem. 
3.1. One node cyclotomic Carter-Payne homomorphisms. We are now ready to prove our main theorem in the special case when $\boldsymbol{\lambda}$ and $\boldsymbol{\mu}$ differ by one node.

Throughout this section, we fix $\boldsymbol{\nu} \in \mathscr{P}_{n+1}^{\Lambda}$ and $i \in I$. If $\boldsymbol{\sigma} \in \mathscr{P}_{n}^{\Lambda}$ write $\boldsymbol{\sigma} \stackrel{i}{\longrightarrow} \boldsymbol{\nu}$ if $\boldsymbol{\sigma} \subset \boldsymbol{\nu}$ and $\operatorname{res}(\boldsymbol{\nu} \backslash \boldsymbol{\sigma})=i$, that is, $\boldsymbol{\nu} \backslash \boldsymbol{\sigma}$ is a removable $i$-node of $\boldsymbol{\nu}$.

3.1. Theorem. Suppose that $\boldsymbol{\lambda}, \boldsymbol{\mu} \stackrel{i}{\longrightarrow} \boldsymbol{\nu}$ with $\boldsymbol{\mu} \triangleright \boldsymbol{\lambda}$. Then

$$
\operatorname{Hom}_{\mathscr{R}_{n}^{\Lambda}}\left(S^{\boldsymbol{\lambda}}\langle a+b\rangle, S^{\boldsymbol{\mu}}\right) \neq 0,
$$

where $a=\#\{\alpha \in \operatorname{Add}(\boldsymbol{\nu}) \mid \operatorname{res}(\alpha)=i$ and $\operatorname{row}(\boldsymbol{\nu} \backslash \boldsymbol{\lambda})<\operatorname{row}(\alpha) \leq \operatorname{row}(\boldsymbol{\nu} \backslash \boldsymbol{\mu})\}$ and $b=\#\{\alpha \in \operatorname{Rem}(\boldsymbol{\nu}) \mid \operatorname{res}(\alpha)=i$ and $\operatorname{row}(\boldsymbol{\nu} \backslash \boldsymbol{\lambda})<\operatorname{row}(\alpha) \leq \operatorname{row}(\boldsymbol{\nu} \backslash \boldsymbol{\mu})\}$. In particular, $a+b>0$.

It is possible, with some persistence, to prove this result algebraically, however, as a limbering-up exercise for the next sections we prove Theorem 3.1 using the braid diagrams introduced in $\$ 2.6$.

If $\boldsymbol{\sigma} \stackrel{i}{\longrightarrow} \boldsymbol{\nu}$, let $\mathfrak{t}_{\boldsymbol{\sigma}}^{\nu}$ be the unique standard $\boldsymbol{\nu}$-tableau such that $\left(\mathfrak{t}_{\boldsymbol{\sigma}}^{\boldsymbol{\nu}}\right)_{\downarrow n}=\mathfrak{t}^{\boldsymbol{\sigma}}$. Then

$$
v_{\mathfrak{t}_{\boldsymbol{\sigma}}^{\nu}}=v_{\mathfrak{t}^{\nu}} \psi_{m} \psi_{m+1} \ldots \psi_{n}
$$

where $m$ is in the same position of $\mathfrak{t}^{\boldsymbol{\nu}}$ as $n+1$ is in $\mathfrak{t}_{\boldsymbol{\sigma}}^{\boldsymbol{\nu}}$. If $\mathfrak{t}$ is any other tableau with Shape $\left(\mathfrak{t}_{\downarrow n}\right)=\operatorname{Shape}\left(\mathfrak{t}_{\boldsymbol{\sigma} \downarrow n}^{\nu}\right)$ then there exists $w \in \mathfrak{S}_{n}$ such that $\mathfrak{t}_{\boldsymbol{\sigma}}^{\boldsymbol{\nu}} w=\mathfrak{t}$ and we define $v_{\mathfrak{t}} \in S^{\nu}$ by $v_{\mathfrak{t}}=v_{\mathfrak{t}^{\nu}} \psi_{w}$. Note that $\psi_{w}$ may depend on the chosen reduced expression for $w$.

Suppose that $\boldsymbol{\sigma}, \boldsymbol{\tau} \stackrel{i}{\longrightarrow} \boldsymbol{\nu}$ and $\boldsymbol{\tau} \triangleright \boldsymbol{\sigma}$. Define $w_{(\boldsymbol{\tau}, \boldsymbol{\sigma})} \in \mathfrak{S}_{n}$ to be the permutation $(m, m-1, \ldots, l)$, where $m$ is the number in position $\boldsymbol{\nu} \backslash \boldsymbol{\tau}$ of $\mathfrak{t}_{\boldsymbol{\sigma}}^{\boldsymbol{\nu}}$ and $l$ is the number in position $\boldsymbol{\nu} \backslash \boldsymbol{\sigma}$ of $\boldsymbol{t}_{\tau}^{\nu}$.

3.2. Lemma. Suppose that $\boldsymbol{\sigma} \stackrel{i}{\longrightarrow} \boldsymbol{\nu}$. Then

$$
v_{\mathfrak{t}_{\boldsymbol{\sigma}}^{\nu}} y_{n+1}=\sum_{\underset{\boldsymbol{\tau}}{\boldsymbol{\tau} \triangleright \boldsymbol{\sigma}} \boldsymbol{\nu}} v_{\mathfrak{t}_{\boldsymbol{\tau}}^{\nu}} \psi_{w_{(\boldsymbol{\tau}, \boldsymbol{\sigma})}} .
$$

Proof. We want to compute $v_{t_{\sigma}^{\nu}} y_{n+1}$ using diagrams. By design this calculation is almost the same as Example 2.20. Nonetheless, we give a detailed explanation of the notation to ensure that the reader understands the diagrams because this language is indispensable later. In terms of diagrams we want to compute:

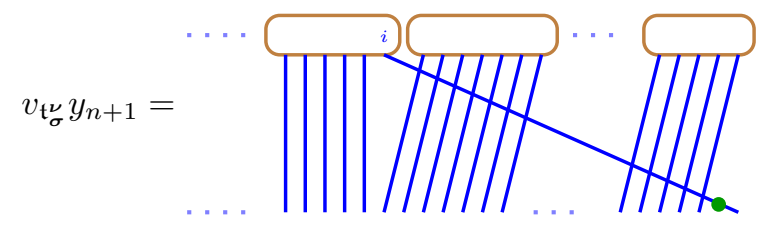

The dot represents $y_{n+1}$ because it is on the $(n+1)$-string, which is the $(n+1)$ th string when we read from left to right along the bottom of the diagram. Reading from top to bottom, the $(n+1)$-string crosses the $k$-string for $k=m, \ldots, n$. Each crossing corresponds to multiplying by some $\psi_{r}$, so $v_{\mathfrak{t}^{\nu}} \psi_{m} \ldots \psi_{n} y_{n+1}=v_{\mathfrak{t}_{\sigma}^{\nu}} y_{n+1}$ as claimed.

To simplify the diagram above we use (2.4) to move the dot past the ${ }^{i} \mathbf{X}^{j}$-crossings above it - here $i$ and $j$ are the residues of the corresponding strings in the diagram. 
If $j \neq i$ then the dot jumps over the crossing without penalty. When $j=i$, however, we have an ${ }^{i} \mathbf{X}^{i}$-crossing and by (2.18).

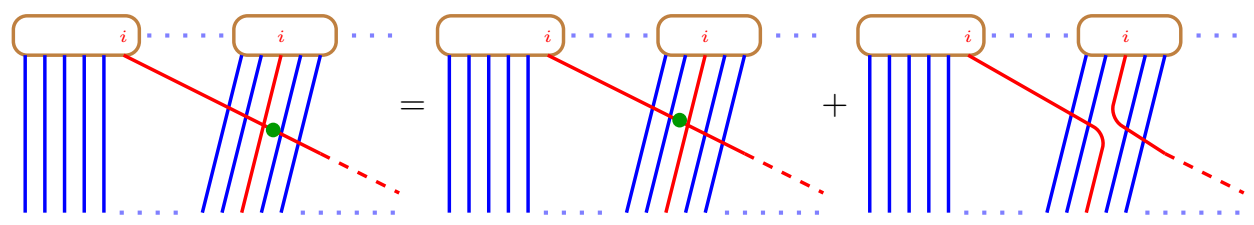

With the first diagram on the right-hand side, we continue moving the dot to the left. With the second diagram there are three possibilities. First, if the rightmost labelled string of residue $i$ does not correspond to a node at the right-hand end of a row of the diagram $\boldsymbol{\nu}$, as drawn in the diagram above, then the diagram is zero by the row relation $(2.17)$ If the node is at the end of row $(l, r)$ and $\nu_{r}^{(l)}=\nu_{r+1}^{(l)}$, where $1 \leq l \leq \ell$, then the diagram is again zero by the Garnir relation (2.19). Otherwise, the right-hand string of residue $i$ moves $n+1$ to a removable node in $\boldsymbol{\nu}$. Let $\boldsymbol{\tau}$ be the multipartition of $n$ obtained by removing this node. Then $\boldsymbol{\tau} \stackrel{i}{\longrightarrow} \boldsymbol{\nu}, \boldsymbol{\tau} \triangleright \boldsymbol{\sigma}$ and the diagram is equal to $v_{\mathfrak{t}_{\tau}^{\nu}} \psi_{w_{(\boldsymbol{\tau}, \boldsymbol{\sigma})}}$.

The lemma follows by repeating this argument and noting that once the dot gets all the way to the top of the diagram we get zero by $(2.17)$.

We now generalise the definitions used in Lemma 3.2 Suppose that $\boldsymbol{\sigma}_{0}, \ldots, \boldsymbol{\sigma}_{z+1}$ are multipartitions of $n$ such that $\boldsymbol{\sigma}_{k} \stackrel{i}{\longrightarrow} \boldsymbol{\nu}$, for $0 \leq k \leq z+1$, ordered so that $\boldsymbol{\sigma}_{z+1} \triangleright \boldsymbol{\sigma}_{z} \triangleright \cdots \triangleright \boldsymbol{\sigma}_{0}$. Define permutations

$$
w_{\left(\boldsymbol{\sigma}_{k}, \boldsymbol{\sigma}_{k-1}, \ldots, \boldsymbol{\sigma}_{0}\right)}=w_{\left(\boldsymbol{\sigma}_{1}, \boldsymbol{\sigma}_{0}\right)} w_{\left(\boldsymbol{\sigma}_{2}, \boldsymbol{\sigma}_{1}\right)} \ldots w_{\left(\boldsymbol{\sigma}_{k}, \boldsymbol{\sigma}_{k-1}\right)},
$$

for $0 \leq k \leq z+1$. Observe that $w_{\left(\boldsymbol{\sigma}_{j+1}, \boldsymbol{\sigma}_{j}\right)} w_{\left(\boldsymbol{\sigma}_{k+1}, \boldsymbol{\sigma}_{k}\right)}=w_{\left(\boldsymbol{\sigma}_{k+1}, \boldsymbol{\sigma}_{k}\right)} w_{\left(\boldsymbol{\sigma}_{j+1}, \boldsymbol{\sigma}_{j}\right)}$, whenever $0 \leq j<k \leq z$. For $0 \leq k \leq z+1$ define $\boldsymbol{\nu}$-tableaux by

$$
\mathfrak{t}_{\left(\boldsymbol{\sigma}_{k}, \boldsymbol{\sigma}_{k-1}, \ldots, \boldsymbol{\sigma}_{0}\right)}=\mathfrak{t}_{\boldsymbol{\sigma}_{k}}^{\nu} w_{\left(\boldsymbol{\sigma}_{k}, \boldsymbol{\sigma}_{k-1}, \ldots, \boldsymbol{\sigma}_{0}\right)} .
$$

Then $\mathfrak{t}_{\left(\boldsymbol{\sigma}_{k}, \boldsymbol{\sigma}_{k-1}, \ldots, \boldsymbol{\sigma}_{0}\right)}$ is standard. Note that $\psi_{w_{\left(\boldsymbol{\sigma}_{k}, \boldsymbol{\sigma}_{k-1}, \ldots, \boldsymbol{\sigma}_{0}\right)}}$ is independent of the choice of reduced expression for $w_{\left(\boldsymbol{\sigma}_{k}, \boldsymbol{\sigma}_{k-1}, \ldots, \boldsymbol{\sigma}_{0}\right)}$, so that

$$
v_{\mathfrak{t}_{\left(\sigma_{k}, \sigma_{k-1}, \ldots, \sigma_{0}\right)}}=v_{\mathfrak{t}_{\boldsymbol{t}_{k}}^{\nu}} \psi_{w_{\left(\boldsymbol{\sigma}_{k}, \boldsymbol{\sigma}_{k-1}, \ldots, \sigma_{0}\right)}} .
$$

3.3. Example. Suppose that $e=3$ and $\kappa=(0,2,0,1)$. Take $n+1=20, \boldsymbol{\nu}=$ $(4,3,1|2| 4,3 \mid 2,1)$ and $i=0$. The removable $i$-nodes are those shaded below

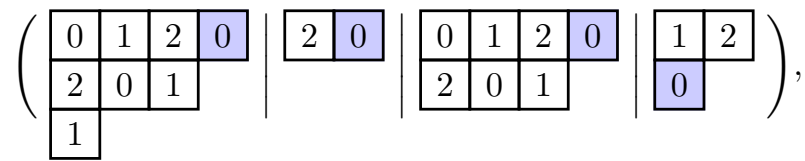

and the partitions $\boldsymbol{\tau}$ such that $\boldsymbol{\tau} \stackrel{i}{\longrightarrow} \boldsymbol{\nu}$ are

$$
\begin{array}{ll}
\boldsymbol{\sigma}_{0}=(3,3,1|2| 4,3 \mid 2,1), & \boldsymbol{\sigma}_{1}=(4,3,1|1| 4,3 \mid 2,1), \\
\boldsymbol{\sigma}_{2}=(4,3,1|2| 3,3 \mid 2,1), & \boldsymbol{\sigma}_{3}=(4,3,1|2| 4,3 \mid 2) .
\end{array}
$$

Then $w_{\left(\boldsymbol{\sigma}_{3}, \boldsymbol{\sigma}_{2}, \boldsymbol{\sigma}_{1}, \boldsymbol{\sigma}_{0}\right)}=(9,8,7,6,5,4)(13,12,11,10)(19,18,17,16,15,14)$ and

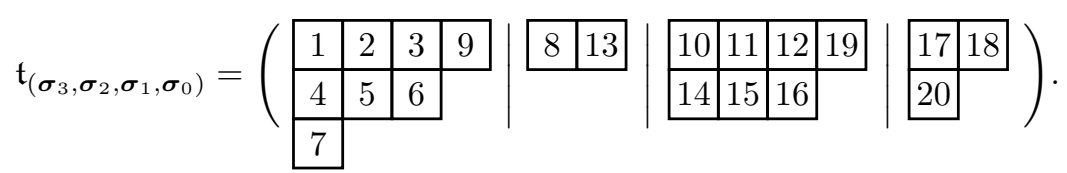


By definition, $w_{\left(\boldsymbol{\sigma}_{k}, \boldsymbol{\sigma}_{k-1}, \ldots, \boldsymbol{\sigma}_{0}\right)} \in \mathfrak{S}_{n}$ so $y_{n+1}$ commutes with $\psi_{w_{\left(\boldsymbol{\sigma}_{k}, \boldsymbol{\sigma}_{k-1}, \ldots, \boldsymbol{\sigma}_{0}\right)}}$, for $0 \leq k \leq z+1$. Hence, Lemma 3.2 immediately implies the following.

3.4. Corollary. Suppose that $\boldsymbol{\sigma} \stackrel{i}{\longrightarrow} \boldsymbol{\nu}$ and that $k \geq 1$. Then

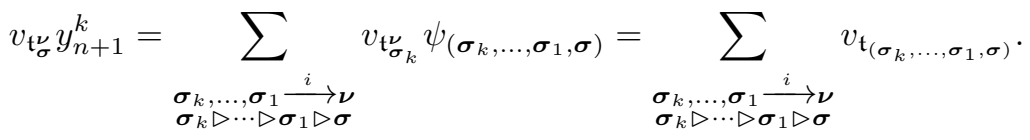

We can now complete the proof of our Main Theorem in the case when $\gamma=1$.

Proof of Theorem 3.1. We remind the reader that the rows of $\boldsymbol{\nu}$ are indexed by pairs $r=($ comp, row $)$, which determine a row in a component of $\boldsymbol{\nu}$. These row indices are totally ordered lexicographically by (2.9)

By definition, $\boldsymbol{\lambda}, \boldsymbol{\mu} \stackrel{i}{\longrightarrow} \boldsymbol{\nu}$ are multipartitions such that $\boldsymbol{\mu} \triangleright \boldsymbol{\lambda}$ where $\boldsymbol{\nu} \backslash \boldsymbol{\lambda}$ is in row $r$ of $\boldsymbol{\nu}$ and $\boldsymbol{\nu} \backslash \boldsymbol{\mu}$ lies in row $s$ for some $r<s$.

Let $\alpha_{0}, \ldots, \alpha_{z+1}$ be the removable $i$-nodes in $\boldsymbol{\nu}$ in rows $r$ to $s$ inclusive, ordered by their row indices. Define multipartitions $\boldsymbol{\lambda}=\boldsymbol{\sigma}_{0}, \boldsymbol{\sigma}_{1}, \ldots, \boldsymbol{\sigma}_{z+1}=\boldsymbol{\mu}$ of $n$ by setting $\boldsymbol{\sigma}_{k}=\boldsymbol{\nu} \backslash\left\{\alpha_{k}\right\}$, for $0 \leq k \leq z+1$. Then $\boldsymbol{\mu}=\boldsymbol{\sigma}_{z+1} \triangleright \boldsymbol{\sigma}_{z} \triangleright \cdots \triangleright \boldsymbol{\sigma}_{0}=\boldsymbol{\lambda}$ and $\boldsymbol{\sigma}_{k} \stackrel{i}{\longrightarrow}$ $\boldsymbol{\nu}$, for $0 \leq k \leq z+1$. Recalling the notation from $\S 2.7$ define $S_{\boldsymbol{\sigma}_{k}}^{\boldsymbol{\nu}}:=\hat{S}_{\boldsymbol{\sigma}_{k}}^{\boldsymbol{\nu}}=\check{S}_{\boldsymbol{\sigma}_{k}}^{\boldsymbol{\nu}}$ and $S_{\mathfrak{t}_{\boldsymbol{\sigma}_{k}}}^{\nu}:=\hat{S}_{\mathfrak{t}_{\boldsymbol{\sigma}_{k}}^{\nu}}^{\nu}=\check{S}_{\mathfrak{t}_{\boldsymbol{\sigma}_{k}}^{\nu}}^{\nu}$, so that $S_{\boldsymbol{\sigma}_{k}}^{\nu}$ has basis $\left\{v_{\mathfrak{t}} \mid \operatorname{row}_{\mathfrak{t}}(n+1)>\operatorname{row}_{\boldsymbol{t}_{\boldsymbol{\sigma}_{k}}}^{\nu}(n+1)\right\}$, for $0 \leq k \leq z+1$. It then follows from (2.23) that the Specht module $S^{\nu}$ has an $\mathscr{R}_{n}^{\Lambda}$-module filtration

$$
S^{\nu} \supseteq S_{\mathfrak{t}_{\lambda}^{\nu}}^{\nu} \supset S_{\lambda}^{\nu} \supseteq S_{\mathfrak{t}_{\mu}^{\nu}}^{\nu} \supset S_{\mu}^{\nu} \supseteq 0 .
$$

Recalling the framework of Lemma 2.24, define an idempotent $e_{n}(i) \in \mathscr{R}_{n+1}^{\Lambda}$ by

$$
e_{n}(i)=\sum_{\left(i_{1}, \ldots, i_{n}\right) \in I^{n}} e\left(i_{1}, \ldots, i_{n}, i\right)
$$

and let $L=e_{n}(i) y_{n+1}^{z+1}$. Then $L$ commutes with $\mathscr{R}_{n}^{\Lambda}$ and $\operatorname{deg} L=2(z+1)$. We claim that multiplication by $L$ induces a non-zero homomorphism from $S^{\boldsymbol{\lambda}}$ to $S^{\mu}$.

Suppose that $x \in S_{\boldsymbol{\lambda}}^{\nu}$. Then $x$ is a $\mathbb{Z}$-linear combination of elements $v_{\mathfrak{t}}$ indexed by standard tableaux $\mathfrak{t}$ such that $\operatorname{row}_{\mathfrak{t}}(n+1)>r$. If $\operatorname{res}_{\mathfrak{t}}(n+1) \neq i$ then $v_{\mathfrak{t}} e_{n}(i)=0$ by $(2.14)$ so that $v_{\mathfrak{t}} L=0$. So suppose that $\operatorname{res}_{\mathfrak{t}}(n+1)=i$. Then $v_{\mathrm{t}} e_{n}(i)=v_{\mathrm{t}}$. If $\operatorname{row}_{\mathfrak{t}}(n+1)>s$ then $\boldsymbol{\mu} \unrhd \mathfrak{t}_{\downarrow n}$ and $v_{\mathfrak{t}} y_{n+1}^{z+1} \in S_{\boldsymbol{\mu}}^{\nu}$ by Lemma 2.16. Otherwise $\operatorname{Shape}\left(\mathfrak{t}_{\downarrow n}\right)=\boldsymbol{\sigma}_{k}$, for some $1 \leq k \leq z+1$. Now $v_{\mathfrak{t}}=v_{\mathfrak{t}_{\boldsymbol{\sigma}}^{\nu}} \psi_{w}$, for $w \in \mathfrak{S}_{n}$ and

$$
v_{\mathfrak{t}} y_{n+1}^{z+1}=v_{\mathfrak{t}_{\boldsymbol{\sigma}_{k}}^{\nu}} \psi_{w} y_{n+1}^{z+1}=v_{\mathfrak{t}_{\boldsymbol{k}}}^{\nu} y_{n+1}^{z+1} \psi_{w} \in S_{\boldsymbol{\sigma}_{z+1}}^{\boldsymbol{\nu}}=S_{\boldsymbol{\mu}}^{\nu}
$$

by Corollary 3.4. Therefore, if $x \in S_{\boldsymbol{\lambda}}^{\nu}$ then $x L \in S_{\boldsymbol{\mu}}^{\nu}$.

Now consider $v_{\mathfrak{t}_{\lambda}^{\nu}} L$. Using Corollary 3.4 again,

$$
v_{\mathfrak{t}_{\lambda}^{\nu}} L=v_{\mathfrak{t}_{\lambda}^{\nu}} y_{n+1}^{z+1} \equiv v_{\mathfrak{t}_{\left(\boldsymbol{\mu}, \boldsymbol{\sigma}_{z}, \ldots, \boldsymbol{\sigma}_{1}, \boldsymbol{\lambda}\right)}} \quad\left(\bmod S_{\boldsymbol{\mu}}^{\boldsymbol{\nu}}\right) .
$$

By construction, $v_{\mathfrak{t}_{\left(\mu, \boldsymbol{\sigma}_{z}, \ldots, \boldsymbol{\sigma}_{1}, \boldsymbol{\lambda}\right)}} \in S_{\mathfrak{t}_{\mu}^{\nu}}^{\nu}$ because $\operatorname{row}_{\mathfrak{t}_{\left(\mu, \boldsymbol{\sigma}_{z}, \ldots, \boldsymbol{\sigma}_{1}, \boldsymbol{\lambda}\right)}}(n+1)=s$ which

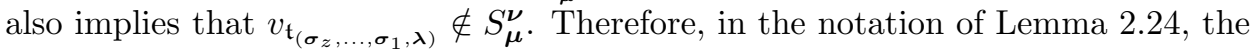
map $\theta_{L}$ induces a non-zero $\mathscr{R}_{n}^{\Lambda}$-module homomorphism from $S^{\boldsymbol{\lambda}}\langle\delta\rangle$ to $S^{\boldsymbol{\mu}}$, where $\delta=\left(\operatorname{deg} \mathfrak{t}_{\boldsymbol{\lambda}}^{\boldsymbol{\nu}}-\operatorname{deg} \mathfrak{t}^{\boldsymbol{\lambda}}\right)-\left(\operatorname{deg} \mathfrak{t}_{\boldsymbol{\mu}}^{\boldsymbol{\nu}}-\operatorname{deg} \mathfrak{t}^{\boldsymbol{\mu}}\right)+\operatorname{deg} L$.

To complete the proof of Theorem 3.1 it remains to show that $\delta$, as defined in the last paragraph, is equal to the number of addable or removable $i$-nodes in rows $r+1$ to $s$. Applying (2.10), $\operatorname{deg} \mathfrak{t}_{\boldsymbol{\lambda}}^{\boldsymbol{\nu}}-\operatorname{deg} \mathfrak{t}^{\boldsymbol{\lambda}}$ is equal to the number of 
addable $i$-nodes minus the number of removable $i$-nodes in $\boldsymbol{\nu}$ which are strictly below row $r$ while $\operatorname{deg} \mathfrak{t}_{\boldsymbol{\mu}}^{\nu}-\operatorname{deg} \mathfrak{t}^{\boldsymbol{\mu}}$ is equal to the number of addable $i$-nodes minus the number of removable $i$-nodes in $\boldsymbol{\nu}$ which are strictly below row $s$. Therefore, $\left(\operatorname{deg} \mathfrak{t}_{\boldsymbol{\lambda}}^{\nu}-\operatorname{deg} \mathfrak{t}^{\boldsymbol{\lambda}}\right)-\left(\operatorname{deg} \mathfrak{t}_{\boldsymbol{\mu}}^{\boldsymbol{\nu}}-\operatorname{deg} \mathfrak{t}^{\boldsymbol{\mu}}\right)$ is the number of addable $i$-nodes minus the number of removable $i$-nodes in $\boldsymbol{\nu}$ in rows $r+1$ to $s$. On the other hand, by definition, $\operatorname{deg} L=2(z+1)$ and $\boldsymbol{\nu}$ has exactly $z+1$ removable $i$-nodes in rows $r+1$ to $s$. This completes the proof.

3.2. Stubborn strings. The proof of Theorem 3.1 is quite straightforward but extending this result to the general case requires several new ideas. We start by introducing stubborn strings which will allow us to prove a significant generalisation of Lemma 3.2. The results and techniques introduced in this section are likely to be of independent interest.

Fix integers $n \geq 0$ and $\gamma>0$ and a multipartition $\boldsymbol{\nu} \in \mathscr{P}_{n+\gamma}^{\Lambda}$. As we will see, when $e=2$ our Main Theorem reduces to Theorem 3.1 so we will assume that $e \neq 2$. This is advantageous because it simplifies relations (2.5) and (2.6)

The basis $\left\{v_{\mathfrak{u}} \mid \mathfrak{u} \in \operatorname{Std}(\boldsymbol{\nu})\right\}$ of the Specht module $S^{\boldsymbol{\nu}}$ defined in $\$ 2.5$ is determined by a choice of reduced expressions and the arguments which follow are easier for certain choices of these reduced expressions. The following lemma, which we leave as an easy exercise, will help us make these choices.

3.5. Lemma. Suppose that $\mathfrak{u} \in \operatorname{Std}(\boldsymbol{\nu})$ and let $\boldsymbol{\sigma}=\operatorname{Shape}\left(\mathfrak{u}_{\downarrow}\right)$. Then there exists a unique tableau $\mathfrak{s} \in \operatorname{Std}(\boldsymbol{\nu})$ such that $\mathfrak{s}_{\downarrow}=\mathfrak{t}^{\boldsymbol{\sigma}}$ and $\mathfrak{s}^{-1}(n+h)=\mathfrak{u}^{-1}(n+h)$, for $1 \leq h \leq \gamma$. Moreover, $\mathfrak{u}=\mathfrak{s} w$, for some $w \in \mathfrak{S}_{n}$ and $\ell(d(\mathfrak{u}))=\ell(d(\mathfrak{s}))+\ell(w)$.

If $\mathfrak{u} \in \operatorname{Std}(\boldsymbol{\nu})$ then write $\mathfrak{u}=\mathfrak{s} w$ as in Lemma 3.5. Then $d(\mathfrak{u})=d(\mathfrak{s}) w$ with $\ell(d(\mathfrak{u}))=\ell(d(\mathfrak{s}))+\ell(w)$. In the sequel we always assume that $v_{\mathfrak{u}}=v_{\mathfrak{s}} \psi_{w}$, for some fixed (and for the moment arbitrary) reduced expressions for $d(\mathfrak{s})$ and $w$.

Let $\mathcal{W}_{n+\gamma}$ be the set of words in $\left\{\psi_{1}, \ldots, \psi_{n+\gamma-1}, y_{1}, \ldots, y_{n+\gamma}\right\}$. We identify a word $\varpi \in \mathcal{W}_{n+\gamma}$ with a braid diagram $B_{\varpi}$ in $S^{\nu}$ by concatenating the diagrams for $v_{\mathfrak{t}^{\nu}}$ and the letters in $\varpi$ the natural way. By definition, $B_{\varpi}$ is a braid diagram which represents an element of $S^{\nu}$. We have not yet attempted to apply the relations to rewrite this element in terms of the basis $\left\{v_{\mathfrak{t}} \mid \mathfrak{t} \in \operatorname{Std}(\boldsymbol{\nu})\right\}$ of $S^{\boldsymbol{\nu}}$.

If $\varpi \in \mathcal{W}_{n+\gamma}$ define $\pi_{\varpi}$ to be the underlying permutation in $\mathfrak{S}_{n+\gamma}$ determined by ignoring any dots on the strings in $B_{\varpi}$. Define $\operatorname{res}(\varpi)=\operatorname{res}\left(\mathfrak{t}^{\nu} \pi_{\varpi}\right)$.

Finally, given $\varpi \in \mathcal{W}_{n+\gamma}$ let $\approx_{\varpi}$ be the equivalence relation on $\{1,2, \ldots, n+\gamma\}$ generated by $l \approx_{\varpi} m$ whenever the $l$-string and $m$-string in $B_{\varpi}$ intersect and have the same residue.

3.6. Proposition. Suppose that $\varpi \in \mathcal{W}_{n+\gamma}$. Then there exist integers $a_{\mathfrak{s}} \in \mathbb{Z}$ such that

$$
v_{\mathfrak{t} \nu} \varpi=\sum_{\mathfrak{s} \in \operatorname{Std}(\boldsymbol{\nu})} a_{\mathfrak{s}} v_{\mathfrak{s}}
$$

where $a_{\mathfrak{s}} \neq 0$ only if $\operatorname{res}(\mathfrak{s})=\operatorname{res}(\varpi)$ and $\left(\pi_{\varpi} d(\mathfrak{s})^{-1}\right)(m) \approx_{\varpi} m$, for $1 \leq m \leq n+\gamma$.

Proof. Since $\left\{v_{\mathfrak{s}} \mid s \in \operatorname{Std}(\boldsymbol{\nu})\right\}$ is a basis of $S^{\boldsymbol{\nu}}$ we can certainly write $v_{\mathfrak{t}} \varpi=$ $\sum_{\mathfrak{s}} a_{s} v_{\mathfrak{s}}$, for some $a_{\mathfrak{s}} \in \mathbb{Z}$. If $\mathbf{i}=\operatorname{res}(\varpi)$ then $v_{\mathfrak{t}^{\nu}} \varpi=v_{\mathfrak{t}^{\nu}} \varpi e(\mathbf{i})$. So $a_{\mathfrak{s}} \neq 0$ only if $\operatorname{res}(\mathfrak{s})=\mathbf{i}$ by $(2.14)$.

To prove the second claim in the lemma observe that if $i \in I$ then all of the KLR relations in Definition 2.2, interpreted in terms of braid diagrams, either preserve or break the ${ }^{i} \mathbf{X}^{i}$-crossings in braid diagrams. In particular, the relations in $\mathscr{R}_{n}^{\Lambda}$ never 
create new ${ }^{i} X^{i}$-crossings in the sense that two strings of the same residue intersect in the diagrams obtained after applying the relations only if they intersected before the relations were applied. Similarly, relations (a)-(c) in Definition 2.12 do not create new ${ }^{i} \mathbf{X}^{i}$-crossings.

Similarly, we claim that the homogeneous Garnir relations in Definition 2.12 (d) do not generate new ${ }^{i} \mathbf{X}^{i}$-crossings for any $i \in I$. When $e=0$ then there is nothing to prove so suppose that $e>0$. Fix a node $A \in \boldsymbol{\nu}$. Then the terms in the corresponding homogeneous Garnir relation are indexed by the tableaux $\left\{\mathrm{T}_{A}^{(e)} d \mid d \in \mathscr{D}_{A}\right\}$. All of these tableaux are standard except for the Garnir tableau $\mathrm{T}_{A}^{(e)} d_{0}$, where $d_{0}$ is the unique element of $\mathscr{D}_{A}$ of maximal length. Inside the Garnir belt, the braid diagram for $d_{0}$ takes the form:

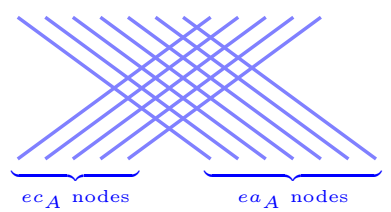

where $a_{A}$ and $c_{A}$ are the number of $e$-bricks in the first and second rows of the Garnir belt, respectively. It follows that if $d \in \mathscr{D}_{a}$ then two strings form an ${ }^{i} \mathbf{X}^{i}$ crossing in the diagram for $\mathrm{T}_{A}^{(e)} d$ only if they form an ${ }^{i} \mathbf{X}^{i}$-crossing in the diagram for $\mathrm{T}_{A}^{(e)} d_{0}$. Consequently, the homogeneous Garnir relations do not create new ${ }^{i} \mathbf{X}^{i}$-crossings, as claimed.

We have shown that none of the relations in $S^{\boldsymbol{\nu}}$ generate new ${ }^{i} \mathbf{X}^{i}$ crossings. Therefore, if $a_{\mathfrak{s}} \neq 0$ and the $l$-string and the $m$-string in $d(\mathfrak{s})$ intersect and have the same residue then $l \approx_{\varpi} m$. Note that $d(\mathfrak{s})^{-1}(m)$ is the number at the top of the $m$-string in the diagram for $\mathfrak{s}$. It follows that $\left(\pi_{\varpi} d(\mathfrak{s})^{-1}\right)(m) \approx_{\varpi} m$ as required.

3.7. Definition. Suppose that $\varpi \in \mathcal{W}_{n+\gamma}$ and that $1 \leq m \leq n+\gamma$. Then the $m$-string is stubborn in $B_{\varpi}$ if $\pi_{\varpi}^{-1}(m) \leq \pi_{\varpi}^{-1}(l)$ for all $l$ such that $l \approx_{\varpi} m$.

An immediate consequence of the definition and Proposition 3.6 is the following.

3.8. Corollary. Suppose that $\varpi \in \mathcal{W}_{n+\gamma}$ and that the $m$-string is stubborn in $B_{\varpi}$. Then

$$
v_{\mathfrak{t}} \varpi=\sum_{\mathfrak{s} \in \operatorname{Std}(\boldsymbol{\nu})} a_{\mathfrak{s}} v_{\mathfrak{s}}
$$

where $a_{\mathfrak{s}} \neq 0$ only if $\operatorname{res}(\mathfrak{s})=\operatorname{res}\left(\mathfrak{t}^{\nu} \pi_{\varpi}\right)$ and $d(\mathfrak{s})^{-1}(m) \geq \pi_{\varpi}^{-1}(m)$.

Define $\operatorname{Std}_{n}(\boldsymbol{\nu})=\left\{\mathfrak{t} \in \operatorname{Std}(\boldsymbol{\nu}) \mid \mathfrak{t}_{\downarrow n}=\mathfrak{t}^{\boldsymbol{\sigma}}\right.$ where $\left.\boldsymbol{\sigma}=\operatorname{Shape}\left(\mathfrak{t}_{\downarrow n}\right)\right\}$. These tableaux have already appeared in Lemma 3.5. We are particularly interested in the following subset of $\operatorname{Std}_{n}(\boldsymbol{\nu})$ : set

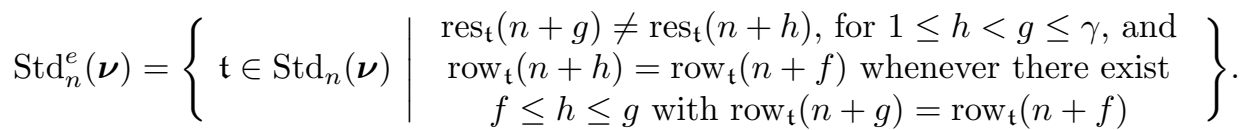

The next result should be compared with Lemma 3.2.

3.9. Lemma. Suppose that $\mathfrak{t} \in \operatorname{Std}_{n}^{e}(\boldsymbol{\nu})$. Fix an integer $g$ with $1 \leq g \leq \gamma$ and suppose $f$ is minimal such that $1 \leq f \leq g$ and $\operatorname{row}_{\mathfrak{t}}(n+f)=\operatorname{row}_{\mathfrak{t}}(n+g)$. Then

$$
v_{\mathfrak{t}} y_{n+g}=\sum_{s \in \operatorname{Std}(\boldsymbol{\nu})} a_{\mathfrak{s}} v_{\mathfrak{s}}
$$


for some integers $a_{\mathfrak{s}}$ such that $a_{\mathfrak{s}} \neq 0$ only if $\operatorname{res}(\mathfrak{s})=\operatorname{res}(\mathfrak{t})$ and

$$
\operatorname{row}_{\mathfrak{s}}(n+h) \geq \operatorname{row}_{\mathfrak{t}}(n+h), \quad \text { for } 1 \leq h \leq \gamma,
$$

and this inequality is strict whenever $f \leq h \leq g$.

Proof. In terms of diagrams, to prove the lemma we need to compute the following:

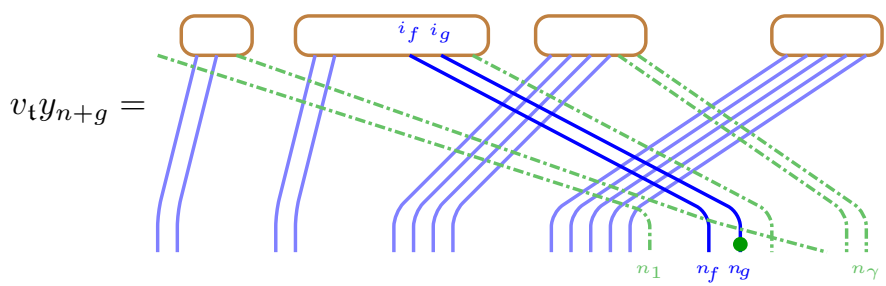

where for convenience we write $n_{h}$ instead of $n+h$ and $i_{h}=\operatorname{res}_{\mathfrak{t}}(n+h)$ for $1 \leq h \leq \gamma$. Thus, the $n_{h}$-string is the string of residue $i_{h}$ that ends at $n+h$ in the bottom row of the diagram. As in the diagram, $n_{f}=n+f$ and $n_{g}=n+g$ are in the same row of $\mathfrak{t}$ and if $m \leq n_{f}$ is in a row of higher index in $\mathfrak{t}$ than $n_{f}$ then the $m$-string must cross the $n_{h}$-string whenever $f \leq h \leq g$. The dashed lines indicate that we do not know (or care) where the $n_{h}$-strings are in the diagram if $h<f$ or if $h>g$. As we will see we can may ignore these lines because $i_{h} \neq i_{g}$, whenever $h \neq g$ and $1 \leq h \leq \gamma$.

By assumption the $(n+h)$-strings, for $1 \leq h \leq \gamma$, have distinct residues so the fact that some of the $(n+h)$-strings might cross the $n+f, \ldots, n+g$ strings will not cause us any difficulties. Exactly as in the proof of Lemma 3.2 we can slide the dot up the $(n+g)$-string until we reach a string of residue $i_{g}=\operatorname{res}_{\mathfrak{t}}(n+g)$. Applying (2.18), and the relation $v_{\mathfrak{t}^{\nu}} y_{r}=0$ for all $1 \leq r \leq n+\gamma$, shows that $v_{\mathfrak{t}} y_{n+g}$ is equal to the sum of diagrams of the form

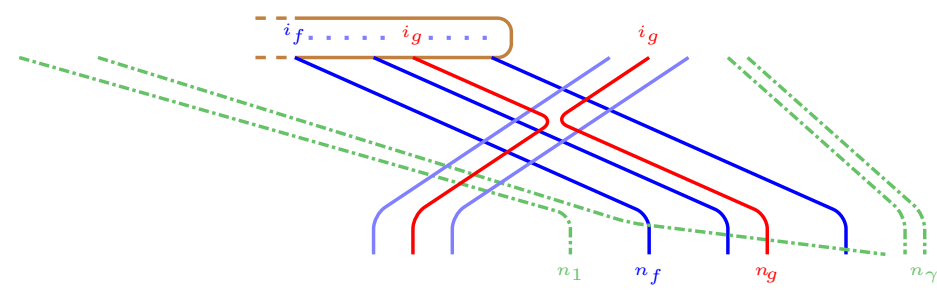

Let $\varpi$ be the word corresponding to one of these diagrams. Noting that the $n+$ $1, \ldots, n+\gamma$ strings all have different residues, we see that for $1 \leq h \leq \gamma$, the $(n+h)$-string is stubborn. Applying Corollary 3.8.

$$
v_{\mathfrak{t} \nu} \varpi=\sum_{\mathfrak{s} \in \operatorname{Std}(\boldsymbol{\nu})} b_{\mathfrak{s}} v_{\mathfrak{s}},
$$

for $b_{\mathfrak{s}} \in \mathbb{Z}$ such that $b_{\mathfrak{s}} \neq 0$ only if $\operatorname{res}(\mathfrak{s})=\operatorname{res}(\mathfrak{t})$ and $d(\mathfrak{s})^{-1}(n+h) \geq \pi_{\varpi}^{-1}(n+h) \geq$ $d(\mathfrak{t})^{-1}(n+h)$, for $1 \leq h \leq \gamma$. In particular, if $b_{\mathfrak{s}} \neq 0$ then $d(\mathfrak{s})^{-1}(n+g)>$ $d(\mathfrak{t})^{-1}(n+g)$. But this implies that $\operatorname{row}_{\mathfrak{s}}(n+g)>\operatorname{row}_{\mathfrak{t}}(n+g)$ because there is no position in $\operatorname{row}_{\mathfrak{t}}(n+g)$ which is to the right of the position containing $n+g$ in $\mathfrak{t}$ and which has the same residue. Similarly, if $f \leq h<g$ then $\operatorname{row}_{\mathfrak{t}}(n+h)=\operatorname{row}_{\mathfrak{t}}(n+g)$. Then $\operatorname{row}_{\mathfrak{s}}(n+h)>\operatorname{row}_{\mathfrak{t}}(n+h)$ because otherwise $\operatorname{row}_{\mathfrak{s}}(n+h)=\operatorname{row}_{\mathfrak{t}}(n+h)$ which implies that the entry in $\mathfrak{s}$ which is in the same position as $n+g$ in $\mathfrak{t}$ is both larger than $n+h$ and has residue $\operatorname{res}_{\mathfrak{t}}(n+g)$, since $\operatorname{res}(\mathfrak{s})=\operatorname{res}(\mathfrak{t})$, which is impossible. 
Hence, $\operatorname{row}_{\mathfrak{s}}(n+h) \geq \operatorname{row}_{\mathfrak{t}}(n+h)$, for $1 \leq h \leq \gamma$, and this inequality is strict if $f \leq h \leq g$. This completes the proof.

We also need the following more specialised version of Lemma 3.9.

3.10. Lemma. Suppose that $\mathfrak{t} \in \operatorname{Std}_{n}^{e}(\boldsymbol{\nu})$. Choose $1 \leq g \leq \gamma$ and suppose $1 \leq f \leq g$ is minimal such that $\operatorname{row}_{\mathfrak{t}}(n+f)=\operatorname{row}_{\mathfrak{t}}(n+g)$. Set $n_{h}=n+h$ and $i_{h}=\operatorname{res}_{\mathfrak{t}}(n+h)$, for $1 \leq h \leq \gamma$. Then $v_{\mathfrak{t}} y_{n+g}$ is the sum of all diagrams of the form

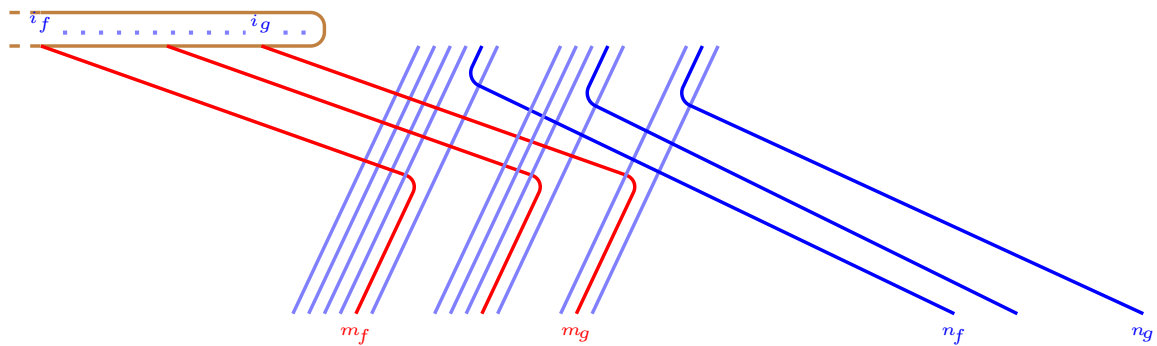

where the sum is over all integers $1 \leq m_{f}<\cdots<m_{g} \leq n$ such that $\operatorname{res}_{\mathfrak{t}}\left(m_{h}\right)=$ $\operatorname{res}_{\mathfrak{t}}(n+h)$ and $\operatorname{row}_{\mathfrak{t}}\left(m_{h}\right)>\operatorname{row}_{\mathfrak{t}}(n+h)$ for $f \leq h \leq g$, and where all other strings appear in the same positions as they appear in $\mathfrak{t}$.

Warning! The notation $m_{h}$ for the integers $m_{f}, \ldots, m_{g}$ in Lemma 3.10 is convenient because $m_{h}$ depends on $n_{h}$, however, the reader should not confuse $m_{h}$ and $m+h$ (since $m$ is not defined) even though $n_{h}=n+h$, for $1 \leq h \leq \gamma$.

Proof. As in the proof of Lemma 3.9, in terms of diagrams of the form

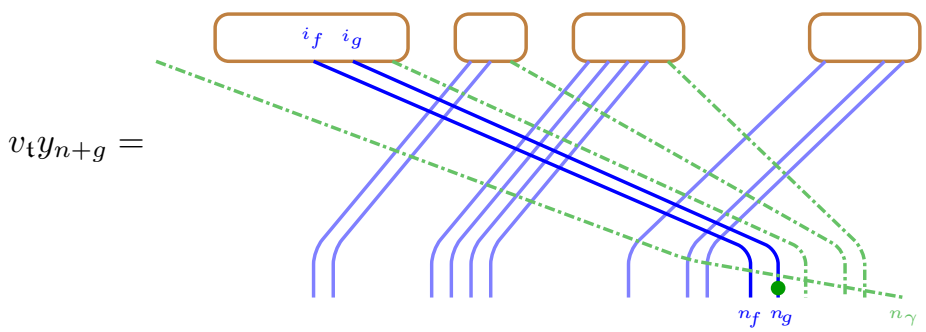

We saw in the proof of Lemma 3.9 that the dashed strings do not move, so for clarity we will now ignore them. As in the proof of Lemma 3.9. sliding the dot up the $(n+g)$-string shows that $v_{\mathfrak{t}} y_{n+g}$ is the sum of all diagrams of the form

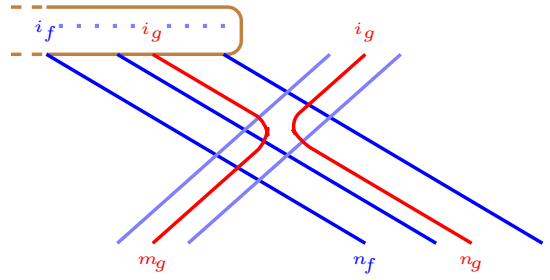

If $f=g$ then this proves the lemma, so we may assume that $f<g$.

Each of these diagrams above corresponds to a tableau $\mathfrak{s}$ where $\mathfrak{s}=\mathfrak{t}\left(m_{g}, n_{g}\right)$ and $\operatorname{res}_{\mathfrak{t}}\left(m_{g}\right)=i_{g}=\operatorname{res}_{\mathfrak{t}}\left(n_{g}\right)$. Note that $m_{g} \leq n$ because the residues of the $n_{h}$-strings are all distinct, for $f \leq h \leq g$. Unfortunately, since $f<g$, the tableau $\mathfrak{s}$ is not standard because $n_{g-1}$ and $m_{g}$ both appear in the same row of $\mathfrak{s}$, with $n_{g-1}$ directly 
to the left of $m_{g}$, and $m_{g}<n_{g-1}$. To prove the lemma we show that this diagram can be written as a linear combination of diagrams which correspond to tableaux of the form $\mathfrak{t} w$, where $w=\left(m_{f}, n_{f}\right) \ldots\left(m_{g}, n_{g}\right) \in \mathfrak{S}_{n+g}$, with $n_{f}, \ldots, n_{g}$ all appearing in later rows of $\mathfrak{t} w$ than $m_{f}, \ldots, m_{g}$. As we will see, the key observation is that each $n_{h}$-string crosses an $m_{h}$ string of the same residue, for $f \leq h \leq g$.

Fix $h$ with $f \leq h \leq g$. We claim that the diagram displayed above is equal to the sum of all diagrams of the form

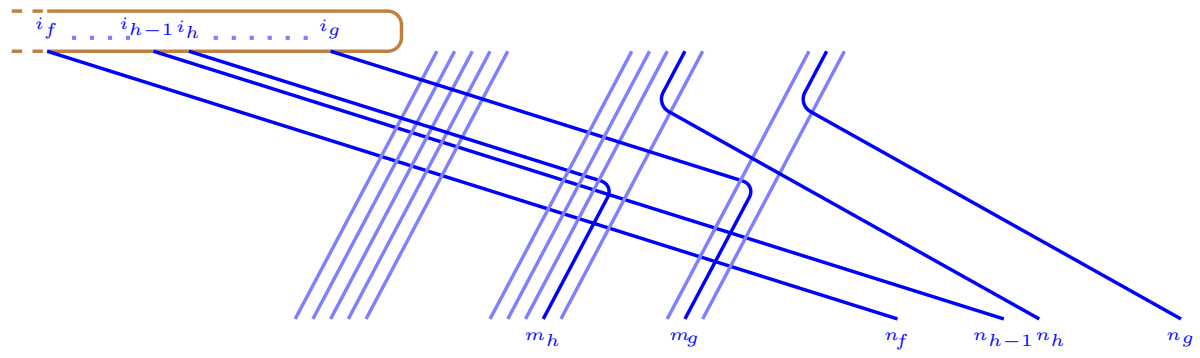

where all other strings are in exactly the same position as they are in the diagram for $\mathfrak{t}$. That is, we have replaced the tableau $\mathfrak{s}=\mathfrak{t}\left(m_{g}, n_{g}\right)$ in the paragraph above with $\mathfrak{t}\left(m_{h}, n_{h}\right) \ldots\left(m_{g}, n_{g}\right)$, for some integers $m_{h}<m_{h+1}<\cdots<m_{g}$ such that $\operatorname{res}_{\mathfrak{t}}\left(m_{k}\right)=\operatorname{res}_{\mathfrak{t}}\left(n_{k}\right)$ and $\operatorname{row}_{\mathfrak{t}}\left(m_{h}\right)>\operatorname{row}_{\mathfrak{t}}\left(n_{h}\right)$, for $h \leq k \leq g$. The sum is over all choices of $m_{h}, \ldots, m_{g}$ subject to these constraints. The lemma is exactly our claim when $h=f$.

We prove our claim by downwards induction on $h$. If $h=g$ then the claim is automatically true. By induction we can assume that $f+1 \leq h \leq g$ and that the claim holds for $h$. For the inductive step we show that the last diagram, where $m_{h}, \ldots, m_{g}$ are fixed, is equal to the sum of all diagrams of the required form with $m_{h-1}<m_{h}$. To do this we want to pull the $m_{h}$-string past the string of residue $i_{h-1}$, which is immediately to the left of this string. Applying relation (2.18), we can keep on doing this until we reach an $i_{h-1}$-crossing by which time we will have a diagram of the form

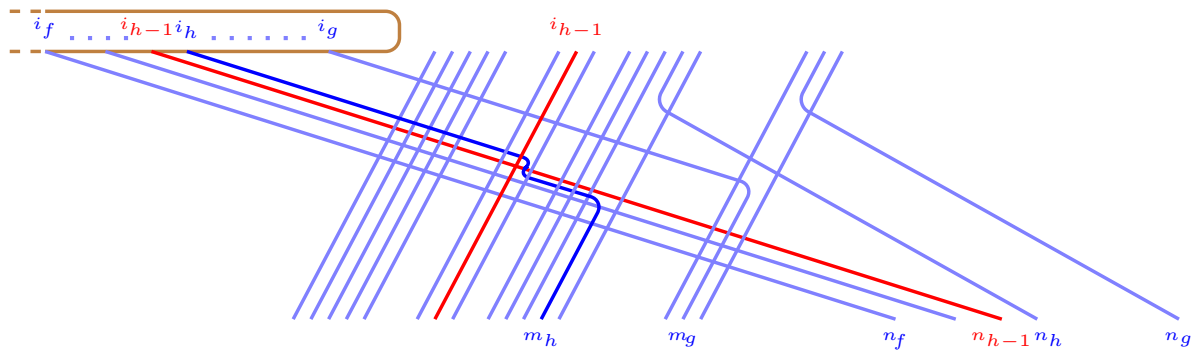

The two strings of residue $i_{h-1}$ and the string of residue $i_{h}$ are now in exactly the configuration where (2.18) gives a non-trivial braid relation. Therefore, when we pull the $m_{h}$-string past the $i_{h-1}$-crossing we obtain two diagrams. The first diagram is obtained by applying the standard three string braid relation, so that we pull the $m_{h}$-string past the $i_{h-1}$-crossing. Therefore, in the first diagram we can keep pulling the $m_{h}$-string to the left until we reach the next $i_{h-1}$-crossing, or until we reach the top of the diagram in which case we get zero by (2.17) 
The second diagram that arises when we apply the braid relation in (2.18) is obtained by cutting the $i_{h-1}$-crossing, vertically, so it has the form

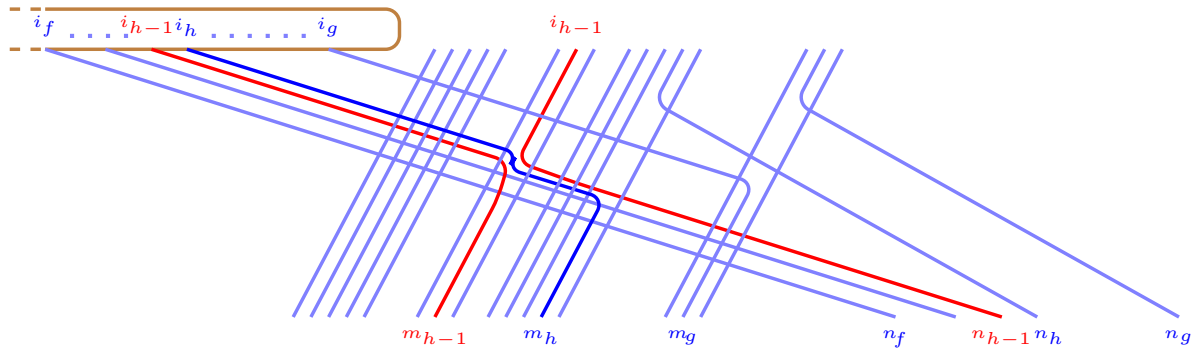

Observe that this diagram is not of the required form because the $n_{h-1}$-string crosses the $m_{k}$-string twice, for $h<k \leq g$. If $h<k \leq g$ then $i_{h-1} \neq i_{k} \pm 1, i_{k}$ because the residues $i_{h+1}, \ldots, i_{g}$ are all consecutive and, by assumption, $g \leq \gamma<e$. Therefore, we can move the $n_{h-1}$-string past the $m_{k}$-strings using the relation $\psi_{r}^{2} e(\mathbf{i})=e(\mathbf{i})$, for $h<k \leq g$. Hence, the last diagram is equal to

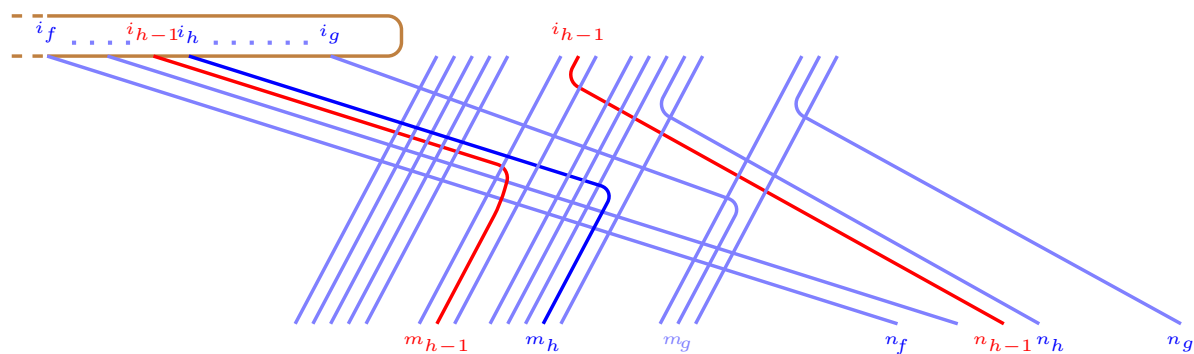

This completes the proof of the inductive step and hence of our claim. Notice that the endpoints of the $n+h, \ldots, n+h$ strings and the strings which start at $i_{h}, \ldots, i_{g}$ have not changed during the proof of the claim. Taking $h=f$ we have proved the lemma.

3.3. One-row cyclotomic Carter-Payne homomorphisms. We now prove the version of our Main Theorem which is closest in spirit to the original Carter-Payne theorem. Even though we are again considering only a special case of our Main Theorem the arguments in this section extend, almost verbatim, to the general case. The proof of our Main Theorem requires considerably more notation, however, to make the argument clearer we consider the "one-row case" first and then highlight the changes needed for the general case.

We continue to assume that $\boldsymbol{\nu}$ is a multipartition of $n+\gamma$ where $n \geq 0$ and $\gamma>0$.

3.11. Definition. Suppose that $j \in I$. A removable $j$-strip of length $\delta$ in $\boldsymbol{\nu}$ is a set of nodes $C=\{(l, r, c),(l, r, c+1), \ldots,(l, r, c+\delta-1)\} \subseteq \boldsymbol{\nu}$ such that $j=\operatorname{res}(l, r, c)$ and $\boldsymbol{\nu} \backslash C$ is a multipartition of $n$. Set $\operatorname{row}(C)=(l, r)$.

That is, a removable $j$-strip in $\boldsymbol{\nu}$ is a set of nodes $C$ which are contained in one row of $\boldsymbol{\nu}$ such that $\boldsymbol{\nu} \backslash C$ is a multipartition and the residue of the leftmost node in $C$ has residue $j$.

If $C$ is a removable $j$-strip in $\boldsymbol{\nu}$ let $\operatorname{res}(C)=\{\operatorname{res}(\alpha) \mid \alpha \in C\}$. If $J \subseteq I$ then a removable $J$-node is a removable $i$-node for some $i \in J$ and an addable $J$-node is an addable $i$-node for some $i \in J$.

The main result of this section is the following: 
3.12. Theorem. Let $\boldsymbol{\lambda}$ and $\boldsymbol{\mu}$ be multipartitions of $n$ and suppose that $\boldsymbol{\mu} \triangleright \boldsymbol{\lambda}$ and that $\boldsymbol{\nu}=\boldsymbol{\lambda} \cup \boldsymbol{\mu}$ is a multipartition of $n+\gamma$ such that $\boldsymbol{\nu} \backslash \boldsymbol{\lambda}$ and $\boldsymbol{\nu} \backslash \boldsymbol{\mu}$ are both removable $j$-strips of length $\gamma$, where $j \in I$ and $\gamma<|I|$. Let $J=\operatorname{res}(\boldsymbol{\nu} \backslash \boldsymbol{\lambda})=\operatorname{res}(\boldsymbol{\nu} \backslash \boldsymbol{\mu})$. Then

$$
\operatorname{Hom}_{\mathscr{R}_{n}^{\Lambda}}\left(S^{\boldsymbol{\lambda}}\langle a-b+2 c\rangle, S^{\boldsymbol{\mu}}\right) \neq 0
$$

where

$$
\begin{aligned}
& a=\#\{\alpha \in \operatorname{Add}(\boldsymbol{\nu}) \mid \operatorname{res}(\alpha) \in J \text { and } \operatorname{row}(\boldsymbol{\nu} \backslash \boldsymbol{\lambda})<\operatorname{row}(\alpha) \leq \operatorname{row}(\boldsymbol{\nu} \backslash \boldsymbol{\mu})\}, \\
& b=\#\{\alpha \in \operatorname{Rem}(\boldsymbol{\nu}) \mid \operatorname{res}(\alpha) \in J \text { and } \operatorname{row}(\boldsymbol{\nu} \backslash \boldsymbol{\lambda})<\operatorname{row}(\alpha) \leq \operatorname{row}(\boldsymbol{\nu} \backslash \boldsymbol{\mu})\}, \\
& c=\#\left\{\begin{array}{l|l}
C \text { is a removable } j \text {-strip of length at most } \gamma \\
\text { such that row }(\boldsymbol{\nu} \backslash \boldsymbol{\lambda})<\operatorname{row}(C) \leq \operatorname{row}(\boldsymbol{\nu} \backslash \boldsymbol{\mu})
\end{array}\right\} .
\end{aligned}
$$

Moreover, $a-b+2 c>0$.

When $\gamma=1$ then it is not hard to see that $b=c$ so that in this case the degree shift in Theorem 3.12 is $a-b+2 c=a+b$, which is strictly positive. More generally, it is easy to see that if $\alpha$ is a removable $i$-node which contributes to the number $b$ in Theorem 3.12 then either $\alpha$ is contained in a removable $j$-strip of length at most $\gamma$ or the row below row $(\alpha)$ has an addable $i$-node for some $i \in J$. As $c>0$ it follows that $a-b+2 c>0$.

In view of the remarks in the last paragraph, Theorem 3.1 is the special case of Theorem 3.12 corresponding to $\gamma=1$. In particular, if $e=2$ then Theorem 3.12 is equivalent to Theorem 3.1. Consequently, as we did in the last section, we can assume that $e \neq 2$ when proving Theorem 3.12 .

For the rest of this section we assume that $\boldsymbol{\lambda}, \boldsymbol{\mu} \subset \boldsymbol{\nu}$ are multipartitions satisfying the assumptions of Theorem 3.12. Once again, the basic strategy for proving Theorem 3.12 is to apply Lemma 2.24. The first step is to set up the notation that we need in order to define a non-zero map from $S^{\boldsymbol{\lambda}}\langle a-b+2 c\rangle$ to $S^{\boldsymbol{\mu}}$.

Recall the definition of $\operatorname{Std}_{n}^{e}(\boldsymbol{\nu})$ from just before Lemma 3.9. Let $\boldsymbol{t}_{\boldsymbol{\lambda}}^{\boldsymbol{\nu}}$ be the unique standard $\boldsymbol{\nu}$-tableau in $\operatorname{Std}_{n}^{e}(\boldsymbol{\nu})$ such that $\operatorname{Shape}\left(\left(\mathfrak{t}_{\boldsymbol{\lambda}}^{\boldsymbol{\nu}}\right)_{\downarrow n}\right)=\boldsymbol{\lambda}$. Then $S^{\boldsymbol{\lambda}} \cong$ $S_{\mathfrak{t}_{\boldsymbol{\lambda}}^{\nu}}^{\boldsymbol{\nu}} / S_{\boldsymbol{\lambda}}^{\boldsymbol{\nu}}\left\langle\operatorname{deg} \mathfrak{t}^{\boldsymbol{\lambda}}-\operatorname{deg} \mathfrak{t}_{\boldsymbol{\lambda}}^{\boldsymbol{\nu}}\right\rangle$ by (2.23). Extending the definition of $\operatorname{Std}_{n}^{e}(\boldsymbol{\nu})$, define

$$
\operatorname{Std}_{\boldsymbol{\lambda}}^{e}(\boldsymbol{\nu})=\left\{\mathfrak{t} \in \operatorname{Std}_{n}^{e}(\boldsymbol{\nu}) \mid \operatorname{res}_{\mathfrak{t}}(n+g)=\operatorname{res}_{\mathfrak{t}_{\boldsymbol{\lambda}}^{\nu}}(n+g), \text { for } 1 \leq g \leq \gamma\right\} .
$$

Note that the residues of the nodes in $\boldsymbol{\nu} \backslash \boldsymbol{\lambda}$ are pairwise distinct. If $\boldsymbol{\sigma} \in \mathscr{P}_{n}^{\Lambda}$, $\boldsymbol{\sigma} \subset \boldsymbol{\nu}$ and $\operatorname{res}(\boldsymbol{\nu} \backslash \boldsymbol{\sigma})=\operatorname{res}(\boldsymbol{\nu} \backslash \boldsymbol{\lambda})$ then define $\mathfrak{t}_{\boldsymbol{\sigma}}^{\boldsymbol{\nu}}=\mathfrak{t}_{\boldsymbol{\sigma}}^{\boldsymbol{\nu}}(\boldsymbol{\lambda})$ to be the unique standard $\boldsymbol{\nu}$-tableau in $\operatorname{Std}_{\boldsymbol{\lambda}}^{e}(\boldsymbol{\nu})$ such that $\left(\mathfrak{t}_{\boldsymbol{\sigma}}^{\nu}\right)_{\downarrow n}=\mathfrak{t}^{\sigma}$.

By assumption, $\boldsymbol{\nu} \backslash \boldsymbol{\lambda}$ and $\boldsymbol{\nu} \backslash \boldsymbol{\mu}$ are both removable $j$-strips where $j=\operatorname{res}_{t_{\boldsymbol{\lambda}}^{\nu}}(n+1)$. Let $C_{0}=\boldsymbol{\nu} \backslash \boldsymbol{\lambda}, C_{1}, \ldots, C_{z+1}=\boldsymbol{\nu} \backslash \boldsymbol{\mu}$ be the removable $j$-strips of length at most $\gamma$ in $\boldsymbol{\nu}$ ordered so that $r_{0}<r_{1}<\cdots<r_{z+1}$, where $r_{k}=\operatorname{row}\left(C_{k}\right)$ for $0 \leq k \leq z+1$. Define standard tableaux $\mathfrak{t}_{0}, \mathfrak{t}_{1}, \ldots, \mathfrak{t}_{z+1}$ recursively by setting $\mathfrak{t}_{0}=\mathfrak{t}_{\boldsymbol{\lambda}}^{\nu}$ and

$$
\mathfrak{t}_{k+1}=\mathfrak{t}_{k} \prod_{h=1}^{g_{k}}\left(\eta_{h}^{(k)}, n+h\right),
$$

where $g_{k}=\# C_{k}$ and $\eta_{h}^{(k)}$ is minimal such that $\operatorname{res}_{\mathfrak{t}_{k}}\left(\eta_{h}^{(k)}\right)=\operatorname{res}_{\mathfrak{t}_{k}}(n+h)$ and

$$
\mathfrak{t}_{k}^{-1}\left(\eta_{h}^{(k)}\right) \in C_{k+1} \cup C_{k+2} \cup \cdots \cup C_{z+1},
$$

for $1 \leq h \leq g_{k}$. Then, $1 \leq \eta_{h}^{(k)} \leq n$ for $1 \leq h \leq g_{k}$.

Before we give an example, we explain the meaning of these definitions. As $j=\operatorname{res}_{t_{\lambda}^{\nu}}(n+1)$, the removable $j$-strips $C_{0}, C_{1}, \ldots, C_{z+1}$ are in precisely those 
rows between $r_{0}$ and $r_{z+1}$ where $n+1$ can appear in a standard tableau with the same residue sequence as $\boldsymbol{t}_{\boldsymbol{\lambda}}^{\nu}$. In such a tableau, if $n+1$ occurs in $C_{k}$ then so does $n+h$ whenever $1 \leq h \leq g_{k}=\# C_{k}$. Finally, the tableau $\mathfrak{t}_{k+1}$ is obtained from $\mathfrak{t}_{k}$ by swapping $n+h$ and $\eta_{h}^{(k)}$, for $1 \leq h \leq g_{k}$, where $\eta_{h}^{(k)}$ is the highest number in $\mathfrak{t}_{k}$ which is below $n+h$, has residue $i_{h}=\operatorname{res}_{\mathfrak{t}_{\lambda}^{\nu}}(n+h)$ and which sits in a removable $j$-strip. That is, the tableaux $\mathfrak{t}_{k}$ are constructed by successively moving $n+1, \ldots, n+\gamma$ into later rows as "slowly" as possible.

3.13. Example. Suppose that $e=3$ and $\kappa=(0,0,1)$. Let $\boldsymbol{\lambda}=(3,3,2,1|2| 3,2)$ and $\boldsymbol{\mu}=(5,3,2,1|2| 3)$ so that $n=16, \gamma=2$ and $\boldsymbol{\nu}=(5,3,2,1|2| 3,2)$. The residues in $\boldsymbol{\nu}$ are

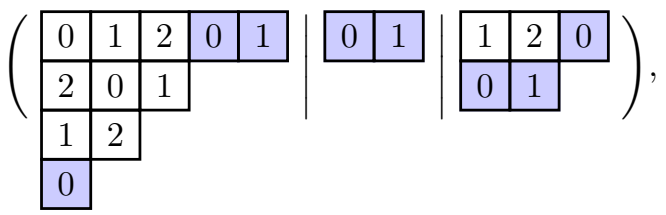

where we have shaded all of the nodes which are contained in a removable 0-strip of length at most 2. Therefore, $z=3$ and our definitions in this case give:

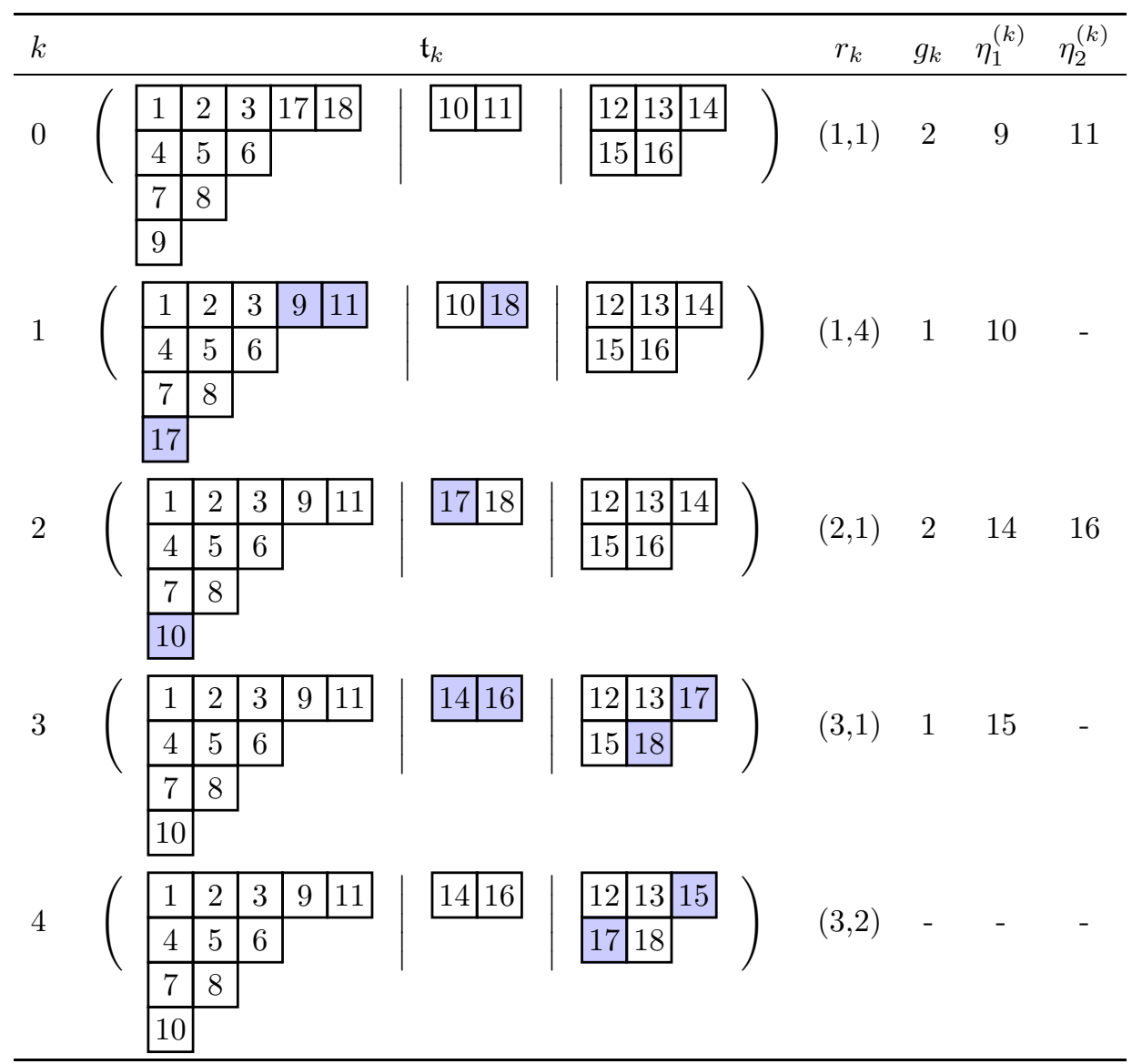

where we have shaded the numbers which have moved at each step. Observe that $\boldsymbol{\mu}=\operatorname{Shape}\left(\left(\mathfrak{t}_{4}\right)_{\downarrow n}\right)$. 
For $0 \leq k \leq z+1$ set $\boldsymbol{\sigma}_{k}=\operatorname{Shape}\left(\left(\mathfrak{t}_{k}\right)_{\downarrow n}\right)$. Then $\boldsymbol{\mu}=\boldsymbol{\sigma}_{z+1} \triangleright \boldsymbol{\sigma}_{z} \triangleright \cdots \triangleright \boldsymbol{\sigma}_{0}=\boldsymbol{\lambda}$. By definition, $\boldsymbol{\sigma}_{k} \in \mathscr{P}_{n}^{\Lambda}, \boldsymbol{\sigma}_{k} \subset \boldsymbol{\nu}$ and $\operatorname{res}\left(\boldsymbol{\nu} \backslash \boldsymbol{\sigma}_{k}\right)=\operatorname{res}(\boldsymbol{\nu} \backslash \boldsymbol{\lambda})$. So, $\mathfrak{t}_{\boldsymbol{\sigma}_{k}}^{\boldsymbol{\nu}} \in \operatorname{Std}_{\boldsymbol{\lambda}}^{e}(\boldsymbol{\nu})$ and we can define permutations $w_{k} \in \mathfrak{S}_{n}$ by $\mathfrak{t}_{k}=\mathfrak{t}_{\boldsymbol{\sigma}_{k}}^{\nu} w_{k}$, for $0 \leq k \leq z+1$.

The next result is a straightforward application of the definitions. Note that part (b) follows by induction on $k$ because if $1 \leq h \leq \gamma$ then, by definition, $n+h$ is contained in a removable $j$-strip. Therefore, $r_{k}=\operatorname{row}_{\mathfrak{t}_{k}}(n+1) \leq \operatorname{row}_{\mathfrak{t}_{k}}(n+h)$ because $\mathfrak{t}_{k}$ is standard, for $0 \leq k \leq z+1$.

3.14. Lemma. Suppose that $0 \leq k \leq z+1$. Then:

a) $\operatorname{row}_{\mathfrak{t}_{k}}(n+h)=r_{k}$ for $1 \leq h \leq g_{k}$.

b) $\operatorname{row}_{\mathfrak{t}_{k}}(n+h)>r_{k}$ for $g_{k}<h \leq \gamma$.

Moreover, $\mathfrak{t}_{z+1} \triangleright \mathfrak{t}_{z} \triangleright \cdots \triangleright \mathfrak{t}_{1} \triangleright \mathfrak{t}_{0}=\mathfrak{t}_{\boldsymbol{\lambda}}^{\nu}$.

We next choose a reduced expression for $w_{k}$ which will be used to fix the basis element $v_{\mathfrak{t}_{k}} \in S^{\boldsymbol{\nu}}$, further specifying the choice made in Lemma 3.5. If $0 \leq k \leq z$ and $1 \leq h \leq g_{k}$ then set

$$
l_{h}^{(k)}=\mathfrak{t}^{\nu}\left(\mathfrak{t}_{k}^{-1}(n+h)\right) \quad \text { and } \quad m_{h}^{(k)}=\mathfrak{t}_{\sigma_{k}}^{\nu}\left(\mathfrak{t}_{k}^{-1}\left(\eta_{h}^{(k)}\right)\right) .
$$

If $1 \leq l \leq m \leq n+\gamma$ let $s(l, m)=(l, l+1)(l+1, l+2) \ldots(m-1, m)$ and set $\psi(l, m)=\psi_{l} \ldots \psi_{m-1}$. Note that $s(m, m)=1$ and $\psi(m, m)=1$.

3.16. Example. Continuing Example 3.13 the permutations $w_{k}$ are:

\begin{tabular}{cccccc}
\hline$k$ & $w_{k}$ & $l_{1}^{(k)}$ & $l_{2}^{(k)}$ & $m_{1}^{(k)}$ & $m_{2}^{(k)}$ \\
\hline 0 & 1 & 4 & 5 & 9 & 11 \\
1 & $s(5,11) s(4,9)$ & 11 & - & 11 & - \\
2 & $s(5,11) s(4,9)$ & 12 & 13 & 14 & 16 \\
3 & $s(13,16) s(12,14) s(5,11) s(4,9)$ & 16 & - & 16 & - \\
4 & $s(13,16) s(12,14) s(5,11) s(4,9)$ & - & - & - & - \\
\hline
\end{tabular}

It might help the reader to keep the last two examples in mind during the proof of the following lemma. The equation in Lemma 3.17 is not as complicated it looks: $g_{k}-h+1$ appears only because we want the right-hand product in the formula to be written in terms of increasing values of $h$.

3.17. Lemma. Suppose that $0 \leq k \leq z$. Then

$$
w_{k+1}=\prod_{h=1}^{g_{k}} s\left(l_{g_{k}-h+1}^{(k)}, m_{g_{k}-h+1}^{(k)}\right) \cdot w_{k} .
$$

Furthermore, $\ell\left(w_{k+1}\right)=\ell\left(w_{k}\right)+\sum_{h^{\prime}=1}^{g_{k}}\left(m_{h^{\prime}}^{(k)}-l_{h^{\prime}}^{(k)}\right)$.

Proof. Directly from the definitions, if $0 \leq k \leq z$ then

$$
\mathfrak{t}_{\sigma_{k+1}}^{\nu} w_{k+1}=\mathfrak{t}_{k+1}=\mathfrak{t}_{k} \prod_{h=1}^{g_{k}}\left(\eta_{h}^{(k)}, n+h\right)=\mathfrak{t}_{\sigma_{k}}^{\nu} w_{k} \prod_{h=1}^{g_{k}}\left(\eta_{h}^{(k)}, n+h\right) .
$$


On the other hand, the definition of $l_{j}^{(k)}$ and $m_{h}^{(k)}$ gives that

$$
\mathfrak{t}_{\sigma_{k}}^{\nu} w_{k} \prod_{h=1}^{g_{k}}\left(\eta_{h}^{(k)}, n+h\right)=\mathfrak{t}_{\sigma_{k+1}}^{\nu} \prod_{h=1}^{g_{k}} s\left(l_{g_{k}-h+1}^{(k)}, m_{g_{k}-h+1}^{(k)}\right) \cdot w_{k} .
$$

Combining these two equations shows that $w_{k+1}=\prod_{h=1}^{g_{k}} s\left(l_{g_{k}-h+1}^{(k)}, m_{g_{k}-h+1}^{(k)}\right) \cdot w_{k}$ as claimed. The formula for $\ell\left(w_{k+1}\right)$ follows by noting that $\ell(s(l, m))=m-l$ if $l \leq m$, that $\ell(w(l, l+1))=\ell(w)+1$ if and only if $w(l)<w(l+1)$ and that

$$
l_{g_{1}}^{(k)}>\ldots>l_{1}^{(k)}>l_{g_{k-1}}^{(k-1)}>\ldots>l_{1}^{(k-1)}>\ldots>l_{g_{1}}^{(1)}>\ldots>l_{1}^{(1)} .
$$

We leave the details to the reader.

Following Lemma 3.17, we fix reduced expressions for $w_{0}, \ldots, w_{z+1}$ by setting $\psi_{w_{0}}=1$ and

$$
\psi_{w_{k+1}}=\prod_{h=1}^{g_{k}} \psi_{s\left(l_{g_{k}-h+1}^{(k)}, m_{g_{k}-h+1}^{(k)}\right)} \psi_{w_{k}},
$$

for $0 \leq k \leq z$. Using this choice of reduced expressions, define

$$
v_{\mathfrak{t}_{k}}=v_{\mathfrak{t}_{\sigma_{k}}^{\nu}} \psi_{w_{k}}
$$

for $0 \leq k \leq z+1$. Notice that the definition of $v_{\mathfrak{t}_{k}}$ is compatible with the choice of reduced expression that we made after Lemma 3.5.

Having set up our notation, we are now ready to start the proof of Theorem 3.12. Define $\boldsymbol{\Psi}_{n}=\left\langle\psi_{1}, \ldots, \psi_{n-1}\right\rangle \subset \mathscr{R}_{n}^{\Lambda}$ to be the (non-unital) subalgebra of $\mathscr{R}_{n}^{\Lambda}$ generated by $\psi_{1}, \ldots, \psi_{n-1}$.

3.19. Lemma. Suppose that $\mathfrak{t} \in \operatorname{Std}_{\boldsymbol{\lambda}}^{e}(\boldsymbol{\nu})$ and that $\operatorname{row}_{\mathfrak{t}}(n+1)=r_{k}$ for some $0 \leq k \leq z$. Let $k \leq l \leq z$. Then

$$
v_{\mathfrak{t}} y_{n+g_{k}} y_{n+g_{k+1}} \ldots y_{n+g_{l}}=\sum_{\mathfrak{s} \in \operatorname{Std}_{\boldsymbol{\lambda}}^{e}(\boldsymbol{\nu})} a_{\mathfrak{s}} v_{\mathfrak{s}} \psi_{\mathfrak{s}},
$$

where $a_{\mathfrak{s}} \in \mathbb{Z}$ and $\psi_{\mathfrak{s}} \in \mathbf{\Psi}_{n}$. Moreover, if $a_{\mathfrak{s}} \neq 0$ then $\operatorname{row}_{\mathfrak{s}}(n+1) \geq r_{l+1}$ and $\operatorname{row}_{\mathfrak{s}}(n+g) \geq \operatorname{row}_{\mathfrak{t}}(n+g)$, for $1 \leq g \leq \gamma$.

Proof. By Lemma 3.5, if $\mathfrak{u} \in \operatorname{Std}(\boldsymbol{\nu})$ and $\operatorname{res}(\mathfrak{u})=\operatorname{res}\left(\mathfrak{t}_{\boldsymbol{\lambda}}^{\nu}\right)$ then $v_{\mathfrak{u}}=v_{\mathfrak{s}} \psi_{w}$ for a unique $\mathfrak{s} \in \operatorname{Std}_{\boldsymbol{\lambda}}^{e}(\boldsymbol{\nu})$ and $w \in \mathfrak{S}_{n}$, so that $v_{\mathfrak{u}} y_{n+g}=v_{\mathfrak{s}} y_{n+g} \psi_{w}$ where $\psi_{w} \in \boldsymbol{\Psi}_{n}$. Observe that $y_{n+g}$ commutes with $\boldsymbol{\Psi}_{n}$, for $1 \leq g \leq \gamma$. Therefore, the lemma follows by applying Lemma 3.9 $(l-k+1)$ times.

Recall from $\S 2.7$ that $\hat{S}_{\boldsymbol{\mu}}^{\nu}=\left\langle v_{\mathfrak{t}}\right| \mathfrak{t} \in \operatorname{Std}(\boldsymbol{\nu})$ and $\boldsymbol{\mu} \unrhd$ Shape $\left.\left(\mathfrak{t}_{\downarrow n}\right)\right\rangle$ is an $\mathscr{R}_{n}^{\Lambda}$ submodule of $S^{\boldsymbol{\nu}}$. For the next result we note that if $\mathfrak{t} \in \operatorname{Std}_{\boldsymbol{\lambda}}^{e}(\boldsymbol{\nu})$ and $r_{0} \leq$ $\operatorname{row}_{\mathfrak{t}}(n+1) \leq r_{z+1}$ then it is not necessarily true that $\mathfrak{t}=\mathfrak{t}_{\boldsymbol{\sigma}_{k}}^{\nu}$, for some $k$ with $0 \leq k \leq z+1$.

3.20. Lemma. Suppose $\mathfrak{t} \in \operatorname{Std}(\boldsymbol{\nu})$, with $\operatorname{res}(\mathfrak{t})=\operatorname{res}\left(\mathfrak{t}_{\boldsymbol{\lambda}}^{\boldsymbol{\nu}}\right)$, and that there exist $0 \leq l \leq z+1$ and $1 \leq g \leq \gamma$ such that $\operatorname{row}_{\mathfrak{t}}(n+1) \geq r_{l}$ and $\operatorname{row}_{\mathfrak{t}}(n+g)>\operatorname{row}_{\mathfrak{t}_{l}}(n+g)$. Then

$$
v_{\mathfrak{t}} \prod_{j=l}^{z} y_{n+g_{j}} \in \hat{S}_{\boldsymbol{\mu}}^{\nu}
$$

where the product is assumed to be equal to 1 if $l=z+1$. 
Proof. First note that if $\operatorname{res}(\mathfrak{t})=\operatorname{res}\left(\mathfrak{t}_{\boldsymbol{\lambda}}^{\nu}\right)$ then by Lemma 3.5, there exist $\mathfrak{s} \in \operatorname{Std}_{\boldsymbol{\lambda}}^{e}(\boldsymbol{\nu})$ and $w \in \mathfrak{S}_{n}$ such that $v_{\mathfrak{t}}=v_{\mathfrak{s}} \psi_{w}$ where $\psi_{w}$ commutes with all the terms in the product. Since $\hat{S}_{\boldsymbol{\mu}}^{\nu}$ is an $\mathscr{R}_{n}^{\Lambda}$-module and $\psi_{w} \in \mathscr{R}_{n}^{\Lambda}$, in order to prove the lemma it is sufficient to consider only the case that $\mathfrak{t} \in \operatorname{Std}_{\boldsymbol{\lambda}}^{e}(\boldsymbol{\nu})$.

Suppose that there exist $0 \leq l \leq z+1$ and $1 \leq g \leq \gamma$ such that row $_{\mathfrak{t}}(n+1) \geq$ $r_{l}$ and $\operatorname{row}_{\mathfrak{t}}(n+g)>\operatorname{row}_{\mathfrak{t}_{l}}(n+g)$. If $\operatorname{row}_{\mathfrak{t}}(n+g)>r_{z+1}$ then by Lemma 3.9. $v_{\mathfrak{t}} \prod_{j=l}^{z} y_{n+g_{j}}$ is a linear combination of basis elements $v_{\mathfrak{a}}$ where row $\mathfrak{a}(n+g)>r_{z+1}$. Then $\boldsymbol{\mu} \nsubseteq$ Shape $\left(\mathfrak{a}_{\downarrow n}\right)$ and so $v_{\mathfrak{a}} \in \hat{S}_{\boldsymbol{\mu}}^{\nu}$. So we may assume that $0 \leq l \leq z$ and that $\operatorname{row}_{\mathfrak{t}}(n+g) \leq r_{z+1}$, that is, $\operatorname{row}_{\mathfrak{t}}(n+g)=r_{k}$ for some $0 \leq k \leq z+1$.

Suppose first that $g=1$, so that $\operatorname{row}_{\mathfrak{t}}(n+1)=r_{k}$ for some $l<k \leq z$. By Lemma 3.19 we can write $v_{\mathfrak{t}} y_{n+g_{k}} \ldots y_{n+g_{z}}$ as a linear combination of terms $v_{\mathfrak{u}} \psi_{\mathfrak{u}}$, where $\mathfrak{u} \in \operatorname{Std}_{\boldsymbol{\lambda}}^{e}(\boldsymbol{\nu}), \psi_{\mathfrak{u}} \in \boldsymbol{\Psi}_{n}$ and $\operatorname{row}_{\mathfrak{u}}(n+1) \geq r_{z+1}$. Then by Lemma 3.9 and Lemma 2.22, $v_{\mathfrak{u}} \psi_{\mathfrak{u}} y_{n+g_{l}} \ldots y_{n+g_{k-1}}$ is a linear combination of terms $v_{\mathfrak{a}}$ where $\mathfrak{a} \in \operatorname{Std}(\boldsymbol{\nu})$ and $\operatorname{row}_{\mathfrak{a}}(n+1)>r_{z+1}$, so that $v_{\mathfrak{a}} \in \hat{S}_{\boldsymbol{\mu}}^{\nu}$.

Now suppose that $2 \leq g \leq \gamma$. By the comments above, we may assume that $\operatorname{row}_{\mathfrak{t}}(n+1)=r_{l} . \quad \operatorname{As}_{\mathfrak{t}} \in \operatorname{Std}_{\boldsymbol{\lambda}}^{e}(\boldsymbol{\nu})$ and $\operatorname{row}_{\mathfrak{t}}(n+1)=r_{l}$ we have $\operatorname{row}_{\mathfrak{t}_{l}}(n+g)>$ $r_{l}$ because $\mathfrak{t} \in \operatorname{Std}_{\boldsymbol{\lambda}}^{e}(\boldsymbol{\nu})$ and $n+g$ is the only number larger than $n+1$ which has residue $\operatorname{res}_{\mathfrak{t}_{l}}(n+g)$. So $\operatorname{row}_{\mathfrak{t}_{l}}(n+g)=r_{k}$ for some $l<k \leq z+1$. By Lemma 3.19, $v_{\mathfrak{t}} y_{n+g_{l}} \ldots y_{n+g_{n+k-1}}$ is equal to a linear combination of terms $v_{\mathfrak{u}} \psi_{\mathfrak{u}}$, where $\mathfrak{u} \in \operatorname{Std}_{\boldsymbol{\lambda}}^{e}(\boldsymbol{\nu}), \psi_{\mathfrak{u}} \in \boldsymbol{\Psi}_{n}, \operatorname{row}_{\mathfrak{u}}(n+1) \geq r_{k}$ and $\operatorname{row}_{\mathfrak{u}}(n+g) \geq \operatorname{row}_{\mathfrak{t}}(n+g)>$ $\operatorname{row}_{\mathfrak{t}_{l}}(n+g)=r_{k}$. However, since $\operatorname{row}_{\mathfrak{u}}(n+g)>r_{k}$ and $\mathfrak{u} \in \operatorname{Std}_{\boldsymbol{\lambda}}^{e}(\boldsymbol{\nu})$ this forces row $_{\mathfrak{u}}(n+1)>r_{k}$ because, again, $n+g$ is the only number larger than $n+1$ with residue $\operatorname{res}_{\mathfrak{t}}(n+g)$. Therefore, $v_{\mathfrak{u}} y_{n+g_{k}} \ldots y_{n+g_{z}} \in \hat{S}_{\boldsymbol{\mu}}^{\nu}$ by the argument above. It follows that $v_{\mathfrak{t}} y_{n+g_{l}} \ldots y_{n+g_{z}} \in \hat{S}_{\boldsymbol{\mu}}^{\nu}$, as required.

The next result, which generalises Lemma 3.2, will show that the map we construct is non-zero.

3.21. Proposition. Suppose that $0 \leq k \leq z$. Then there exist integers $a_{\mathfrak{s}}$ such that

$$
v_{\mathfrak{t}_{k}} y_{n+g_{k}}=v_{\mathfrak{t}_{k+1}}+\sum_{\mathfrak{s} \in \operatorname{Std}(\boldsymbol{\nu})} a_{\mathfrak{s}} v_{\mathfrak{s}}
$$

where $a_{\mathfrak{s}} \neq 0$ only if $\operatorname{res}(\mathfrak{s})=\operatorname{res}\left(\mathfrak{t}_{\boldsymbol{\lambda}}^{\nu}\right), \operatorname{row}_{\mathfrak{s}}(n+1) \geq r_{k+1}$ and either there exists $1 \leq g \leq g_{k}$ with $\operatorname{row}_{\mathfrak{s}}(n+g)>\operatorname{row}_{\mathfrak{t}_{k+1}}(n+g)$ or $\mathfrak{t}_{z+1} \not \mathfrak{s}($ or both $)$.

Proof. It follows from Lemma 2.16 that $v_{\mathfrak{t}_{k}} y_{n+g_{k}}=\sum_{\mathfrak{u} \in \operatorname{Std}(\boldsymbol{\nu})} b_{\mathfrak{u}} v_{\mathfrak{u}}$ where $b_{\mathfrak{u}} \neq 0$ only if $\operatorname{res}(\mathfrak{u})=\operatorname{res}\left(\mathfrak{t}_{k}\right)=\operatorname{res}\left(\mathfrak{t}_{\boldsymbol{\lambda}}^{\nu}\right)$. By construction, $v_{\mathfrak{t}_{k}} y_{n+g_{k}}=v_{\mathfrak{t}_{\boldsymbol{\sigma}}}^{\nu} \psi_{w_{k}} y_{n+g_{k}}=$ $v_{\mathfrak{t}_{\boldsymbol{\sigma}_{k}}^{\nu}} y_{n+g_{k}} \psi_{w_{k}}$, which, by Lemma 3.9 and Lemma 2.22 is a linear sum of basis elements $v_{\mathfrak{u}}$ where $\mathfrak{u} \in \operatorname{Std}(\nu)$ is such that $\operatorname{row}_{\mathfrak{u}}(n+1) \geq r_{k+1}$.

It remains to show that $b_{\mathfrak{t}_{k+1}}=1$ and that if $\mathfrak{u} \neq t_{k+1}$ and $b_{\mathfrak{u}} \neq 0$ then either there exists $1 \leq g \leq g_{k}$ such that $\operatorname{row}_{\mathfrak{u}}(n+g)>\operatorname{row}_{\mathfrak{t}_{k+1}}(n+g)$ or $\mathfrak{t}_{z+1} \not \mathfrak{u}$. Note that the last two cases are not mutually exclusive, however, $\mathfrak{t}_{k+1}$ does not satisfy either of these conditions. 
By Lemma 3.10, $v_{\mathfrak{t}_{\boldsymbol{\sigma}_{k}}^{\nu}} y_{n+g_{k}}$ is equal to the sum of all diagrams of the form

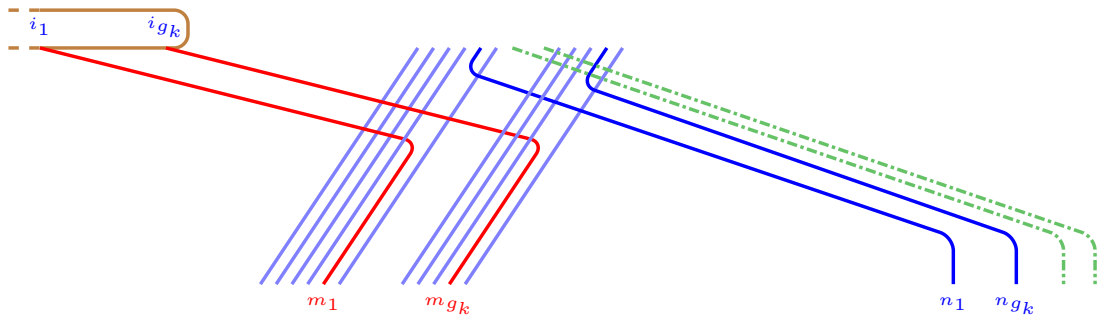

where the sum is over all integers $1 \leq m_{1}<\cdots<m_{g_{k}} \leq n$ such that $\operatorname{res}_{\boldsymbol{t}_{\boldsymbol{\sigma}_{k}}^{\nu}}\left(m_{h}\right)=$ $\operatorname{res}_{\boldsymbol{t}_{\boldsymbol{\sigma}_{k}}^{\nu}}(n+h)$ and $\operatorname{row}_{\boldsymbol{t}_{\boldsymbol{\sigma}_{k}}^{\nu}}\left(m_{h}\right)>\operatorname{row}_{\mathfrak{t}_{\boldsymbol{\sigma}_{k}}^{\nu}}(n+h)$, for $1 \leq h \leq g_{k}$, and where all other strings appear in the same positions that they do in $\mathfrak{t}_{\boldsymbol{\sigma}_{k}}^{\nu}$.

Fix $m_{1}<\cdots<m_{g_{k}}$, as in the last paragraph, and let $\mathbf{m}=\left(m_{1}, \ldots, m_{g_{k}}\right)$. Let $\varpi_{\mathbf{m}} \in \mathcal{W}_{n+\gamma}$ be the word in $\mathcal{W}_{n+\gamma}$ determined by the braid diagram corresponding to $\mathbf{m}$, as drawn above, and let $\pi_{\varpi_{m}}$ be the corresponding permutation. Then we need to compute $v_{\mathfrak{t}^{\nu}} \varpi_{\mathbf{m}} \psi_{w_{k}}$, for all $\mathbf{m}$.

First, suppose that $m_{h}=m_{h}^{(k)}$, for $1 \leq h \leq g_{k}$. Then by Lemma 3.17 and the discussion following it, we have

$$
v_{\mathfrak{t}^{\nu}} \varpi_{\mathbf{m}} \psi_{w_{k}}=v_{\mathfrak{t}_{\boldsymbol{\sigma}_{k+1}}^{\nu}} \prod_{h=1}^{g_{k}} \psi_{s\left(l_{g_{k}-h+1}^{(k)}, m_{g_{k}-h+1}^{(k)}\right)} \psi_{w_{k}}=v_{\mathfrak{t}_{k+1}} .
$$

This implies that $v_{\mathfrak{t}_{k+1}}$ appears in $v_{\mathfrak{t}_{k}} y_{n+g_{k}}$ with coefficient 1 because, as we will see, $v_{\mathfrak{t}_{k+1}}$ only appears in $v_{\mathfrak{t}^{\nu}} \varpi_{\mathbf{m}} \psi_{w_{k}}$ when $\mathbf{m}=\left(m_{1}^{(k)}, \ldots, m_{g_{k}}^{(k)}\right)$.

Now suppose that there exists $1 \leq g \leq g_{k}$ with $m_{g}>m_{g}^{(k)}$. Note that the $(n+g)$ string is stubborn in $\varpi_{\mathbf{m}}$. By Corollary 3.8, $v_{\mathfrak{t}} \varpi_{\mathbf{m}}$ is a linear combination of basis elements $v_{\mathfrak{a}}$ indexed by standard $\boldsymbol{\nu}$-tableaux $\mathfrak{a}$ such that $d(\mathfrak{a})^{-1}(n+g) \geq \pi_{\varpi_{\mathfrak{m}}}^{-1}(n+g)$ and $\operatorname{res}_{\mathfrak{a}}(n+g)=\operatorname{res}_{\mathfrak{t}_{\boldsymbol{\lambda}}^{\nu}}(n+g)$, implying that $\operatorname{row}_{\mathfrak{a}}(n+g)>\operatorname{row}_{\mathfrak{t}_{k+1}}(n+g)$. By Lemma 2.22 $v_{\mathfrak{a}} \psi_{w_{k}}$ is a linear combination of terms $v_{\mathfrak{u}}$ where $\mathfrak{u} \in \operatorname{Std}(\boldsymbol{\nu})$ is such that $\operatorname{row}_{\mathfrak{u}}(n+g)>\operatorname{row}_{\mathfrak{t}_{k+1}}(n+g)$.

Finally, suppose that $m_{h} \leq h^{(k)}$, for $1 \leq h \leq g_{k}$, and that there exists $1 \leq g \leq g_{k}$ with $m_{g}<m_{g}^{(k)}$. Without loss of generality, we may assume $g$ is minimal with this property. In particular, note that $m_{g_{k}} \leq m_{g_{k}}^{(k)}$ so that none of the $n+1, \ldots, n+\gamma$ strings cross. As in the proof of Lemma 3.17.

$$
\mathfrak{t}^{\nu} \pi_{\varpi_{\mathbf{m}}} w_{k}=\mathfrak{t}_{k} \prod_{h=1}^{g_{k}}\left(\eta_{h}, n+h\right),
$$

where $\eta_{h}=\mathfrak{t}_{k}\left(\left(\mathfrak{t}_{\boldsymbol{\sigma}_{k}}^{\nu}\right)^{-1}\left(m_{h}\right)\right)$ and $\ell\left(\pi_{\varpi_{\mathbf{m}}}\right)+\ell\left(w_{k}\right)=\ell\left(\pi_{\varpi_{\mathbf{m}}} w_{k}\right)$. Let $\mathfrak{t}_{\mathbf{m}}=\mathfrak{t}^{\nu} \pi_{\varpi_{\mathbf{m}}} w_{k}$. Suppose first that $\mathfrak{t}_{\mathbf{m}}$ is not row standard. By (3.22), there exists $1 \leq h \leq g_{k}$ such that $n+h$ is immediately to the left of an entry $s$ in $\mathfrak{t}_{m}$, where $1 \leq s \leq n$. Then $v_{\mathfrak{t}^{\nu}} \varpi_{\mathbf{m}}$ is zero by Definition 2.12 (c). Now suppose that $\boldsymbol{t}_{\mathbf{m}}$ is row standard but not column standard. Note that $\operatorname{row}_{\mathfrak{t}_{m}}(n+f) \leq \operatorname{row}_{\mathfrak{t}_{\mathbf{m}}}(n+h)$ for $1 \leq f \leq h \leq \gamma$. By (3.22), there exists $h$ with $1 \leq h \leq \gamma$ such that $n+h$ lies above an entry $s$ in $\mathfrak{t}_{\mathbf{m}}$, for some $1 \leq s \leq n$. But then $v_{\mathfrak{t}^{\nu}} \varpi_{\mathbf{m}}=0$ by (2.19) since either $e=0$ or $\gamma<e$.

So we may assume $\mathfrak{t}_{\mathbf{m}}$ is standard. Let $x=\mathfrak{t}_{\mathbf{m}}\left(\mathfrak{t}_{k}^{-1}(n+g)\right)$, so that $x$ occupies the same position in $\mathfrak{t}_{\mathbf{m}}$ that $n+g$ occupies in $\mathfrak{t}_{k}$. Note that $d\left(\mathfrak{t}_{\mathbf{m}}\right)\left(x^{\prime}\right)=d\left(\mathfrak{t}_{z+1}\right)\left(x^{\prime}\right)$ 
for $1 \leq x^{\prime}<x$. Thus Shape $\left(\left(\mathfrak{t}_{z+1}\right)_{\downarrow x}\right) \not \operatorname{Shape}\left(\left(\mathfrak{t}_{\mathbf{m}}\right)_{\downarrow x}\right)$ and so $\mathfrak{t}_{z+1} \unrhd \mathfrak{t}_{\mathbf{m}}$. Applying Lemma 2.15 $v_{\mathfrak{t}}^{\nu} \varpi_{\mathbf{m}} \psi_{w_{k}}$ is a linear combination of basis elements $v_{\mathfrak{u}}$ where $\mathfrak{u} \in \operatorname{Std}(\boldsymbol{\nu})$ and $\mathfrak{u} \unrhd \mathfrak{t}_{\mathbf{m}}$, so that $\mathfrak{t}_{z+1} \unrhd \mathfrak{u}$.

We are now ready to start proving Theorem 3.12. Recalling the framework of Lemma 2.24, define

$$
L_{\boldsymbol{\mu}}^{\boldsymbol{\lambda}}=y_{n+g_{0}} y_{n+g_{1}} \ldots y_{n+g_{z}} e_{\boldsymbol{\mu}}^{\boldsymbol{\lambda}}, \quad \text { where } \quad e_{\boldsymbol{\mu}}^{\boldsymbol{\lambda}}=\sum_{\mathbf{j} \in I^{n}} e\left(j_{1}, \ldots, j_{n}, i_{1}, \ldots, i_{\gamma}\right)
$$

and $i_{h}=\operatorname{res}_{t_{\lambda}^{\nu}}(n+h)$, for $1 \leq h \leq \gamma$. Then $L_{\boldsymbol{\mu}}^{\boldsymbol{\lambda}}$ commutes with $\mathscr{R}_{n}^{\Lambda}$, so the map

$$
\theta_{\boldsymbol{\mu}}^{\boldsymbol{\lambda}}: S^{\boldsymbol{\nu}} \longrightarrow S^{\boldsymbol{\nu}} ; \quad x \mapsto x L_{\boldsymbol{\mu}}^{\boldsymbol{\lambda}}, \quad \text { for } x \in S^{\boldsymbol{\nu}},
$$

is an $\mathscr{R}_{n}^{\Lambda}$-module endomorphism of $S^{\nu}$.

3.24. Example. Let $\boldsymbol{\lambda}$ and $\boldsymbol{\mu}$ be as in Example 3.13 and Example 3.16. Then

$$
\theta_{\boldsymbol{\mu}}^{\boldsymbol{\lambda}}(x)=x y_{17}^{2} y_{18}^{2} \sum_{\mathbf{j} \in I^{n}} e\left(j_{1}, \ldots, j_{n}, 0,1\right)
$$

for all $x \in S^{\nu}$.

In the notation of Theorem 3.12 $a=3, b=5$ and $c=4$, so that $a-b+2 c=6$ and, consequently, there exists a non-zero homomorphism $S^{\boldsymbol{\lambda}}\langle 6\rangle \rightarrow S^{\boldsymbol{\mu}}$ by Theorem 3.12, We will show that $\theta_{\boldsymbol{\mu}}^{\boldsymbol{\lambda}}$ induces such a map.

Proof of Theorem 3.12. We follow the same basic strategy that we used to prove Theorem 3.1 that is, we engineer the circumstances that we need to apply Lemma 2.24. By (2.23), $S^{\boldsymbol{\nu}}$ has an $\mathscr{R}_{n}^{\Lambda}$-module filtration

$$
S^{\nu} \supseteq \check{S}_{\mathfrak{t}_{\lambda}^{\nu}}^{\nu} \supset \check{S}_{\boldsymbol{\lambda}}^{\nu} \supseteq \hat{S}_{\mathfrak{t}_{\mu}^{\nu}}^{\nu} \supset \hat{S}_{\boldsymbol{\mu}}^{\nu} \supset 0
$$

where $\check{S}_{\mathfrak{t}_{\boldsymbol{\lambda}}^{\nu}}^{\nu} / \check{S}_{\boldsymbol{\lambda}}^{\nu} \cong S^{\boldsymbol{\lambda}}\left\langle\operatorname{deg} \mathfrak{t}_{\boldsymbol{\lambda}}^{\nu}-\operatorname{deg} \mathfrak{t}^{\boldsymbol{\lambda}}\right\rangle$ and $\hat{S}_{\mathfrak{t}_{\boldsymbol{\mu}}^{\nu}}^{\nu} / \hat{S}_{\boldsymbol{\mu}}^{\nu} \cong S^{\boldsymbol{\mu}}\left\langle\operatorname{deg} \mathfrak{t}_{\boldsymbol{\mu}}^{\nu}-\operatorname{deg} \mathfrak{t}^{\boldsymbol{\mu}}\right\rangle$. By Lemma 2.24 in order to show that $\theta_{\mu}^{\boldsymbol{\lambda}}$ induces a map $S^{\boldsymbol{\lambda}}\langle\delta\rangle \rightarrow S^{\boldsymbol{\mu}}$, for some $\delta \in \mathbb{Z}$, it is enough to prove the following three statements:
(A) $\theta_{\boldsymbol{\mu}}^{\boldsymbol{\lambda}}\left(\check{S}_{\boldsymbol{\lambda}}^{\nu}\right) \subseteq \hat{S}_{\boldsymbol{\mu}}^{\nu}$
(B) $\theta_{\boldsymbol{\mu}}^{\boldsymbol{\lambda}}\left(\check{S}_{\mathfrak{t}_{\lambda}^{\nu}}^{\nu}\right) \subseteq \hat{S}_{\mathfrak{t}_{\mu}^{\nu}}^{\nu}$
and
(C) $\theta_{\boldsymbol{\mu}}^{\boldsymbol{\lambda}}\left(v_{\mathrm{t}_{\lambda}^{\nu}}\right) \notin \hat{S}_{\boldsymbol{\mu}}^{\nu}$.

First consider (A). Since $\theta_{\boldsymbol{\mu}}^{\boldsymbol{\lambda}}$ is an $\mathscr{R}_{n}^{\Lambda}$-homomorphism it is enough to show that $\theta_{\boldsymbol{\mu}}^{\boldsymbol{\lambda}}\left(v_{\mathfrak{t}}\right) \in \hat{S}_{\mathfrak{t}_{\boldsymbol{\mu}}^{\nu}}^{\nu}$ whenever $\mathfrak{t} \in \operatorname{Std}_{n}(\boldsymbol{\nu})$ and $\operatorname{Shape}\left(\mathfrak{t}_{\downarrow n}\right) \triangleright \boldsymbol{\lambda}$. If $\mathfrak{t} \notin \operatorname{Std}_{\boldsymbol{\lambda}}^{e}(\boldsymbol{\nu})$ then $\theta_{\boldsymbol{\mu}}^{\boldsymbol{\lambda}}\left(v_{\mathfrak{t}}\right)=0$ because $v_{\mathfrak{t}} e_{\boldsymbol{\mu}}^{\boldsymbol{\lambda}}=0$ by (2.14). Therefore, we may assume that $\mathfrak{t} \in \operatorname{Std}_{\boldsymbol{\lambda}}^{e}(\boldsymbol{\nu})$. Since Shape $\left(\mathfrak{t}_{\downarrow n}\right) \triangleright \boldsymbol{\lambda}$, we have $\operatorname{row}_{\mathfrak{t}}(n+h) \geq r_{0}$ for $1 \leq h \leq \gamma$ with at least one of these equalities being strict, so since $\operatorname{res}(\mathfrak{t})=\operatorname{res}\left(\mathfrak{t}_{\boldsymbol{\lambda}}^{\nu}\right)$ we have $\operatorname{row}_{\mathfrak{t}}(n+1)>r_{0}$. Setting $g=1$ and $l=0$ in Lemma 3.20 $\theta_{\boldsymbol{\mu}}^{\boldsymbol{\lambda}}\left(v_{\mathfrak{t}}\right) \in \hat{S}_{\boldsymbol{\mu}}^{\nu}$ so property (A) holds.

By definition, $\theta_{\boldsymbol{\mu}}^{\boldsymbol{\lambda}}\left(v_{\mathfrak{t}_{\lambda}^{\nu}}\right)=v_{\mathfrak{t}_{\lambda}^{\nu}} y_{n+g_{0}} \ldots y_{n+g_{z}}$. To prove (B) and (C) we would like to argue by induction on $k$ by considering the elements $v_{t_{\lambda}^{\nu}} y_{n+g_{0}} \ldots y_{n+g_{k}}$, where $0 \leq k \leq z$. The difficulty is that when we write $v_{\mathfrak{t}_{\lambda}^{\nu}} y_{n+g_{0}} \ldots y_{n+g_{k}}=\sum_{\mathfrak{t}} a_{\mathfrak{t}} v_{\mathfrak{t}}$ then it can happen that $a_{\mathfrak{t}} \neq 0$ when $\operatorname{row}_{\mathfrak{t}}(n+h)<\operatorname{row}_{\mathfrak{t}_{z}}(n+1)$ for some $h$ with $1 \leq h \leq \gamma$. To cater for this we have to argue slightly circuitously. For $0 \leq k \leq z+1$ define

$$
\operatorname{Std}_{\boldsymbol{\lambda}}(\boldsymbol{\nu}, k)=\left\{\mathfrak{u} \in \operatorname{Std}(\boldsymbol{\nu}) \mid \operatorname{res}(\mathfrak{u})=\operatorname{res}\left(\mathfrak{t}_{\boldsymbol{\lambda}}^{\nu}\right), \operatorname{row}_{\mathfrak{u}}(n+1)=r_{k} \text { and } \mathfrak{t}_{z+1} \not \mathfrak{u}\right\} .
$$

To complete the proof we claim that if $0 \leq k \leq z+1$ then

$$
v_{\mathfrak{t}_{\boldsymbol{\lambda}}^{\nu}} y_{n+g_{0}} \ldots y_{n+g_{z}} \equiv\left(v_{\mathfrak{t}_{k}}+\sum_{\mathfrak{u} \in \operatorname{Std}_{\boldsymbol{\lambda}}(\boldsymbol{\nu}, k)} a_{\mathfrak{u}} v_{\mathfrak{u}}\right) y_{n+g_{k}} \ldots y_{n+g_{z}} \quad\left(\bmod \hat{S}_{\boldsymbol{\mu}}^{\nu}\right),
$$


for some $a_{\mathfrak{u}} \in \mathbb{Z}$. By definition, $v_{\mathfrak{t}_{z+1}} \in \hat{S}_{\mathfrak{t}_{\mu}^{\nu}}^{\nu}$ and $v_{\mathfrak{t}_{z+1}} \notin \hat{S}_{\boldsymbol{\mu}}^{\nu}$. Moreover, $v_{\mathfrak{u}} \in \hat{S}_{\mathfrak{t}_{\mu}^{\nu}}^{\nu}$ for all $\mathfrak{u} \in \operatorname{Std}_{\boldsymbol{\lambda}}(\boldsymbol{\nu}, z+1)$. Therefore, if $k=z+1$ then $(3.25)$ is the statement

$$
\theta_{\boldsymbol{\mu}}^{\boldsymbol{\lambda}}\left(v_{\mathfrak{t}_{\boldsymbol{\lambda}}^{\nu}}\right) \equiv v_{\mathfrak{t}_{z+1}} \quad\left(\bmod \hat{S}_{\mathfrak{t}_{\mu}^{\nu}}^{\nu}\right)
$$

So, taking $k=z+1$ in (3.25) establishes (B) and (C) and completes the proof.

To prove that (3.25) holds we argue by induction on $k$. If $k=0$ then there is nothing to prove because we can set $a_{\mathfrak{u}}=0$ for all $\mathfrak{u} \in \operatorname{Std}_{\boldsymbol{\lambda}}(\boldsymbol{\nu}, 0)$. Hence, by induction it is enough to show that if (3.25) holds for $k+1$ whenever it holds for $k$. First, suppose that $\mathfrak{u} \in \operatorname{Std}_{\boldsymbol{\lambda}}(\boldsymbol{\nu}, k)$ and that $a_{\mathfrak{u}} \neq 0$ in (3.25). Using Lemma 3.5, we can write $v_{\mathfrak{u}}=v_{\mathfrak{s}} \psi_{w}$ where $\mathfrak{s} \in \operatorname{Std}_{n}^{e}(\boldsymbol{\nu})$ and $w \in \mathfrak{S}_{n}$. By Lemma 3.9 and Lemma 2.22, $v_{\mathfrak{u}} y_{n+g_{k}}=v_{\mathfrak{s}} \psi_{w} y_{n+g_{k}}=v_{\mathfrak{s}} y_{n+g_{k}} \psi_{w}$ is a $\mathbb{Z}$-linear combination of terms $v_{\mathfrak{t}}$ where $\mathfrak{t} \in \operatorname{Std}(\boldsymbol{\nu})$ and $\operatorname{row}_{\mathfrak{t}}(n+1)>r_{k}$. Therefore, $\operatorname{row}_{\mathfrak{t}}(n+1) \geq r_{k+1}$ since $n+1$ can only appear in a removable $j$-strip of length at most $\gamma$. If $\operatorname{row}_{\mathfrak{t}}(n+1)>r_{k+1}$ then $v_{\mathfrak{t}} y_{n+g_{k+1}} \ldots y_{n+g_{z}} \in \hat{S}_{\boldsymbol{\mu}}^{\nu}$ by Lemma 3.20. Alternatively, if $\operatorname{row}_{\mathfrak{t}}(n+1)=r_{k+1}$ then $\mathfrak{t}_{z+1} \unrhd \mathfrak{t}$, because $\mathfrak{t}_{z+1} \unrhd \mathfrak{u}$ and $\mathfrak{t} \triangleright \mathfrak{u}$ by Lemma 2.16. Therefore, $\mathfrak{t} \in \operatorname{Std}_{\boldsymbol{\lambda}}(\boldsymbol{\nu}, k+1)$.

Now consider the leading term $\left(v_{\mathfrak{t}_{k}} y_{n+g_{k}}\right) y_{n+g_{k+1}} \ldots y_{n+g_{z}}$ appearing in (3.25). By Proposition 3.21, $v_{\mathfrak{t}_{k}} y_{n+g_{k}}$ is equal to $v_{\mathfrak{t}_{k+1}}$ plus a $\mathbb{Z}$-linear combination of terms $v_{\mathfrak{t}}$ such that $\operatorname{row}_{\mathfrak{t}}(n+1) \geq r_{k+1}$ and either $\mathfrak{t}_{z+1} \unrhd \mathfrak{t}$, or there exists a $g$ such that $\operatorname{row}_{\mathfrak{t}}(n+g)>\operatorname{row}_{\mathfrak{t}_{k+1}}(n+g)$ and $1 \leq g \leq g_{k}$. In the latter case, $v_{\mathfrak{t}} y_{n+g_{k+1}} \ldots y_{n+g_{z}} \in$ $\hat{S}_{\boldsymbol{\mu}}^{\boldsymbol{\nu}}$ by Lemma 3.20. On the other hand, if $\operatorname{row}_{\mathfrak{t}}(n+1)=r_{k+1}$ and $\mathfrak{t}_{z+1} \unrhd \mathfrak{t}$ then $\mathfrak{t} \in \operatorname{Std}_{\boldsymbol{\lambda}}(\boldsymbol{\nu}, k+1)$.

We have now shown that $(3.25)$ holds for $k+1$ and so proved our claim. Therefore, by Lemma 2.24, $\theta_{\boldsymbol{\mu}}^{\boldsymbol{\lambda}}$ induces a non-zero homogeneous map $S^{\boldsymbol{\lambda}}\langle\delta\rangle \rightarrow S^{\boldsymbol{\mu}}$, where $\delta=\left(\operatorname{deg} \mathfrak{t}_{\boldsymbol{\lambda}}^{\boldsymbol{\nu}}-\operatorname{deg} \mathfrak{t}^{\boldsymbol{\lambda}}\right)-\left(\operatorname{deg} \mathfrak{t}_{\boldsymbol{\mu}}^{\boldsymbol{\nu}}-\operatorname{deg} \mathfrak{t}^{\boldsymbol{\mu}}\right)+\operatorname{deg} L_{\boldsymbol{\mu}}^{\boldsymbol{\lambda}}$. Arguing as in the last paragraph of the proof of Theorem 3.1 shows that $\delta=a-b+2 c$, where $a, b$ and $c$ are as in the statement of Theorem 3.12 We leave these details to the reader.

3.4. Cyclotomic Carter-Payne pairs. We now turn to the statement and proof of our Main Theorem. Special cases of our main result include Theorem 3.1 and Theorem 3.12, both of which motivate our main result and simplify its proof.

If $\boldsymbol{\lambda}$ and $\boldsymbol{\nu}$ are multipartitions and $\boldsymbol{\lambda} \subset \boldsymbol{\nu}$ let $\operatorname{res}(\boldsymbol{\nu} \backslash \boldsymbol{\lambda})=\{\operatorname{res}(\alpha) \mid \alpha \in \boldsymbol{\nu} \backslash \boldsymbol{\lambda}\}$. If $J \subseteq I$ and $i, j \in J$ write $j \leq_{J} i$ if $\{j, j+1, \ldots, i\} \subseteq J$.

3.26. Definition. Suppose that $\boldsymbol{\lambda}$ and $\boldsymbol{\mu}$ are multipartitions of $n \geq 0$ and let $\boldsymbol{\nu}=\boldsymbol{\lambda} \cup \boldsymbol{\mu}$ and $J=\operatorname{res}(\boldsymbol{\nu} \backslash \boldsymbol{\lambda})$. Then $(\boldsymbol{\lambda}, \boldsymbol{\mu})$ is a cyclotomic Carter-Payne pair if $J=\operatorname{res}(\boldsymbol{\nu} \backslash \boldsymbol{\mu}),|J|=|\boldsymbol{\nu} \backslash \boldsymbol{\lambda}|$ and $|J|<|I|$, and if $i, j \in J$ and $j \leq_{J} i$ then

$$
\operatorname{row} \alpha_{\boldsymbol{\lambda}}^{\nu}(j) \leq \operatorname{row} \alpha_{\boldsymbol{\lambda}}^{\nu}(i), \quad \operatorname{row} \alpha_{\boldsymbol{\mu}}^{\nu}(j)=\operatorname{row} \alpha_{\boldsymbol{\mu}}^{\nu}(i) \quad \text { and } \quad \operatorname{row} \alpha_{\boldsymbol{\lambda}}^{\nu}(i) \leq \operatorname{row} \alpha_{\boldsymbol{\mu}}^{\nu}(i) \text {, }
$$

and $\alpha_{\boldsymbol{\lambda}}^{\nu}(i)$ is in a removable $j$-strip. Here, and below, if $k \in J$ then $\alpha_{\boldsymbol{\lambda}}^{\nu}(k) \in \boldsymbol{\nu} \backslash \boldsymbol{\lambda}$ and $\alpha_{\boldsymbol{\mu}}^{\boldsymbol{\nu}}(k) \in \boldsymbol{\nu} \backslash \boldsymbol{\mu}$ are the unique nodes in $\boldsymbol{\nu}$ such that $\operatorname{res} \alpha_{\boldsymbol{\lambda}}^{\nu}(k)=k=\alpha_{\boldsymbol{\mu}}^{\nu}(k)$.

Note that in Definition 3.26 the residues in the set $J$ are necessarily distinct because $|J|=|\boldsymbol{\nu} \backslash \boldsymbol{\lambda}|$. It is easy to see that if $\boldsymbol{\lambda}$ and $\boldsymbol{\mu}$ are multipartitions satisfying the assumptions of Theorem 3.12 then $(\boldsymbol{\lambda}, \boldsymbol{\mu})$ is a cyclotomic Carter-Payne pair.

3.27. Example. Suppose that $e=0$ and let $\kappa=(0,4,0,0,4)$. Let $\boldsymbol{\lambda}=(\emptyset|\emptyset| 2|3| 1)$ and $\boldsymbol{\mu}=(2|1| 3|\emptyset| \emptyset)$ so that $\boldsymbol{\nu}=\boldsymbol{\lambda} \cup \boldsymbol{\mu}=(2|1| 3|3| 1)$. In the first diagram below, we have shaded the nodes $\alpha_{\boldsymbol{\lambda}}^{\nu}(i)$ and in the second we have shaded the nodes $\alpha_{\boldsymbol{\mu}}^{\nu}(i)$, 
for $i \in J=\{0,1,2,4\}$ :

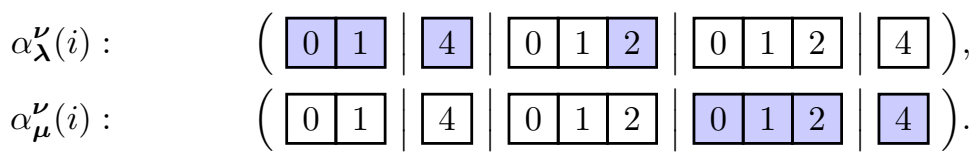

Hence, $(\boldsymbol{\lambda}, \boldsymbol{\mu})$ is a cyclotomic Carter-Payne pair.

The aim of this section is to prove the following result, which is the most general statement of our Main Theorem from the introduction. To state this result we continue to use the notation from Definition 3.26

3.28. Theorem. Suppose that $(\boldsymbol{\lambda}, \boldsymbol{\mu})$ is a cyclotomic Carter-Payne pair and let $J=\operatorname{res}(\boldsymbol{\nu} \backslash \boldsymbol{\lambda})$ and $J^{*}=\{j \in J \mid j-1 \notin J\}$. Then

$$
\operatorname{Hom}_{\mathscr{R}_{n}^{\Lambda}}\left(S^{\boldsymbol{\lambda}}\langle a-b+2 c-d\rangle, S^{\boldsymbol{\mu}}\right) \neq 0,
$$

where for $j \in J^{*}$ we set $\gamma_{j}=\#\left\{i \in J \mid j \leq_{J} i\right\}$ and

$$
\begin{aligned}
& a=\#\left\{\alpha \in \operatorname{Add}(\boldsymbol{\nu}) \mid \operatorname{res}(\alpha)=i \in J \text { and } \operatorname{row} \alpha_{\boldsymbol{\lambda}}^{\nu}(i)<\operatorname{row} \alpha \leq \operatorname{row} \alpha_{\boldsymbol{\mu}}^{\nu}(i)\right\}, \\
& b=\#\left\{\alpha \in \operatorname{Rem}(\boldsymbol{\nu}) \mid \operatorname{res}(\alpha)=i \in J \text { and } \operatorname{row} \alpha_{\boldsymbol{\lambda}}^{\nu}(i)<\operatorname{row} \alpha \leq \operatorname{row} \alpha_{\boldsymbol{\mu}}^{\nu}(i)\right\}, \\
& c=\#\left\{C \mid \begin{array}{c}
C \text { is a removable } j \text {-strip of length at most } \gamma_{j} \text { such } \\
\text { that row } \alpha_{\boldsymbol{\lambda}}^{\nu}(j)<\operatorname{row} C \leq \operatorname{row} \alpha_{\boldsymbol{\mu}}^{\nu}(j), \text { for } j \in J^{*}
\end{array}\right\}, \\
& d=\#\left\{\operatorname{row} \alpha_{\boldsymbol{\lambda}}^{\nu}(i) \mid i \in J\right\}-\#\left\{\operatorname{row} \alpha_{\boldsymbol{\mu}}^{\nu}(i) \mid i \in J\right\} .
\end{aligned}
$$

Moreover, $a-b+2 c-d>0$.

When $e=2$, Theorem 3.28 reduces to Theorem 3.1 (and Theorem 3.12). When $e=3$, Theorem 3.28 is slightly stronger than Theorem 3.12 because the nodes in $\boldsymbol{\nu} \backslash \boldsymbol{\lambda}$ are not necessarily in the same row.

By Definition 3.26, if $j \leq_{J} i$ then $\operatorname{row} \alpha_{\boldsymbol{\mu}}^{\nu}(i)=\operatorname{row} \alpha_{\boldsymbol{\mu}}^{\nu}(j)$ but $\alpha_{\boldsymbol{\lambda}}^{\nu}(i)$ and $\alpha_{\boldsymbol{\lambda}}^{\nu}(j)$ can be in different rows. Therefore, the integer $d$ in Theorem 3.28 is the sum over $j \in J^{*}$ of the differences between the number of rows occupied by the nodes $\left\{\alpha_{\boldsymbol{\lambda}}^{\nu}(i) \mid j \leq_{J} i\right\}$ and the nodes $\left\{\alpha_{\boldsymbol{\mu}}^{\nu}(i) \mid j \leq_{J} i\right\}$. Every row that contributes to this sum has, for some $j \in J^{*}$, a removable $j$-strip $C$ of length at most $\gamma_{j}$ where row $\alpha_{\boldsymbol{\lambda}}^{\nu}(j)<\operatorname{row} C \leq \operatorname{row} \alpha_{\boldsymbol{\mu}}^{\nu}(j)$. Such rows also contribute towards the integer $c$ (but not the integer $b$ ). The argument given in the proof of Theorem 3.12 then shows that $a-b+2 c-d>0$.

By the last paragraph, if $\boldsymbol{\nu} \backslash \boldsymbol{\lambda}$ is contained in one row then $d=0$ and Theorem 3.28 reduces to Theorem 3.12. More generally, as we will see, the proof of Theorem 3.28 is almost identical to the proof of Theorem 3.12. For this reason, we only sketch the proof of Theorem 3.28, highlighting those places where changes to the previous argument are needed.

We now fix a cyclotomic Carter-Payne pair $(\boldsymbol{\lambda}, \boldsymbol{\mu})$ and turn to the proof of Theorem 3.28. Note that if $e=2$ then $(\boldsymbol{\lambda}, \boldsymbol{\mu})$ is a cyclotomic Carter-Payne pair only if $\gamma=1$, in which case Theorem 3.28 reduces to Theorem 3.1. We can therefore assume for the rest of this section that $e \neq 2$.

To prove Theorem 3.28 we generalise the construction of the tableaux $\mathfrak{t}_{k}$ and the multipartitions $\boldsymbol{\sigma}_{k}$, for $0 \leq k \leq z+1$, which were used in the proof of Theorem 3.12. We invite the reader to check that these two notations agree in the special case when the pair $(\boldsymbol{\lambda}, \boldsymbol{\mu})$ satisfies the assumptions of Theorem 3.12. 
As in Theorem 3.28, set $\boldsymbol{\nu}=\boldsymbol{\lambda} \cup \boldsymbol{\mu}, J=\operatorname{res}(\boldsymbol{\nu} \backslash \boldsymbol{\lambda})$ and $J^{*}=\{j \in J \mid j-1 \notin J\}$. If $j \in J^{*}$ define $\gamma_{j}=\#\left\{i \in J \mid j \leq_{J} i\right\}$ and let $\gamma=\sum_{j \in J^{*}} \gamma_{j}$. Then $\gamma=|\boldsymbol{\nu} \backslash \boldsymbol{\lambda}|$.

Suppose that $\mathfrak{t}_{\boldsymbol{\lambda}}^{\boldsymbol{\nu}}$ is a standard $\boldsymbol{\nu}$-tableau such that $\left(\mathfrak{t}_{\boldsymbol{\lambda}}^{\boldsymbol{\nu}}\right)_{\downarrow n}=\mathfrak{t}^{\boldsymbol{\lambda}}$ and if $i, i+1 \in J$ and row $\alpha_{\boldsymbol{\lambda}}^{\nu}(i)=\operatorname{row} \alpha_{\boldsymbol{\lambda}}^{\nu}(i+1)$ then $\mathfrak{t}_{\boldsymbol{\lambda}}^{\nu}\left(\alpha_{\boldsymbol{\lambda}}^{\nu}(i+1)\right)=\mathfrak{t}_{\boldsymbol{\lambda}}^{\nu}\left(\alpha_{\boldsymbol{\lambda}}^{\nu}(i)\right)+1$, and let

$$
\operatorname{Std}_{\boldsymbol{\lambda}}^{e}(\boldsymbol{\nu})=\left\{\mathfrak{t} \in \operatorname{Std}_{n}^{e}(\boldsymbol{\nu}) \mid \operatorname{res}_{\mathfrak{t}}(n+g)=\operatorname{res}_{\mathfrak{t}_{\boldsymbol{\lambda}}^{\nu}}(n+g), \text { for } 1 \leq g \leq \gamma\right\} .
$$

The choice of $\mathfrak{t}_{\boldsymbol{\lambda}}^{\nu}$ does affect our definition of the $\mathscr{R}_{n}^{\Lambda}$-modules $\check{S}_{\mathfrak{t}_{\lambda}^{\nu}}^{\nu}, \hat{S}_{\mathfrak{t}_{\mu}^{\nu}}^{\nu} \subset S^{\nu}$, and consequently the definition of the $\mathscr{R}_{n}^{\Lambda}$-endomorphism $\theta_{\mu}^{\boldsymbol{\lambda}}: S^{\boldsymbol{\nu}} \rightarrow S^{\boldsymbol{\nu}}$, defined in (3.29) below, but it does not affect the statement of the Main Theorem.

As before, if $\boldsymbol{\sigma} \in \mathscr{P}_{n}^{\Lambda}$ is a multipartition such that $J=\operatorname{res}(\boldsymbol{\nu} \backslash \boldsymbol{\sigma})$ and $\boldsymbol{\sigma} \subset \boldsymbol{\nu}$ define $\mathfrak{t}_{\boldsymbol{\sigma}}^{\nu}=\mathfrak{t}_{\boldsymbol{\sigma}}^{\boldsymbol{\nu}}(\boldsymbol{\lambda})$ to be the unique standard $\boldsymbol{\nu}$-tableau in $\operatorname{Std}_{\boldsymbol{\lambda}}^{e}(\boldsymbol{\nu})$ such that $\left(\mathfrak{t}_{\boldsymbol{\sigma}}^{\boldsymbol{\nu}}\right)_{\downarrow n}=\mathfrak{t}^{\boldsymbol{\sigma}}$.

Mirroring the definitions in $\S 3.3$, let $r$ be the smallest row index, with respect to the lexicographic order (2.9), such that the corresponding rows of $\boldsymbol{\nu}$ and $\boldsymbol{\lambda}$ are different and let $s$ be the largest row index such that $\boldsymbol{\nu}$ and $\boldsymbol{\mu}$ differ. Let $C_{0}, C_{1}, \ldots, C_{z+1}$ be the complete list of the removable $j$-strips of length at most $\gamma_{j}$, for some $j \in J^{*}$, lying between rows $r$ and $s$ in the diagram of $\boldsymbol{\nu}$ such that $r=$ $r_{0}<r_{1}<\cdots<r_{z+1}=s$, where $r_{k}=\operatorname{row}\left(C_{k}\right)$ for $0 \leq k \leq z+1$. For $0 \leq k \leq z+1$ define $j_{k} \in J^{*}$ to be the residue such that $C_{k}$ is a removable $j_{k}$-strip and let $f_{k}$ be the unique integer such that $\operatorname{res}_{t_{\lambda}^{\nu}}\left(n+f_{k}\right)=j_{k}$ and $1 \leq f_{k} \leq \gamma$. Almost exactly as before, define standard tableaux $\mathfrak{t}_{0}, \mathfrak{t}_{1}, \ldots, \mathfrak{t}_{z+1}$ recursively by letting $\mathfrak{t}_{0}=\mathfrak{t}_{\lambda}^{\nu}$ and setting

$$
\mathfrak{t}_{k+1}=\mathfrak{t}_{k} \prod_{h=f_{k}}^{g_{k}}\left(\eta_{h}^{(k)}, n+h\right),
$$

where $f_{k} \leq g_{k} \leq \gamma$ and $g_{k}$ is maximal such that $\operatorname{row}_{\mathfrak{t}_{k}}\left(n+g_{k}\right)=r_{k}$ and the integer $\eta_{h}^{(k)}$ is minimal such that $\operatorname{res}_{\mathfrak{t}_{k}}\left(\eta_{h}^{(k)}\right)=\operatorname{res}_{\mathfrak{t}_{k}}(n+h)$ and

$$
\mathfrak{t}_{k}^{-1}\left(\eta_{h}^{(k)}\right) \in C_{k+1} \cup C_{k+2} \cup \cdots \cup C_{z+1},
$$

for $f_{k} \leq h \leq g_{k}$. By construction, $\operatorname{row}_{\mathfrak{t}_{k}}\left(n+f_{k}\right)=r_{k}$, for $0 \leq k \leq z+1$.

Generalising (3.23)] define $\theta_{\boldsymbol{\mu}}^{\boldsymbol{\lambda}}$ to be the $\mathscr{R}_{n}^{\Lambda}$-endomorphism of $S^{\boldsymbol{\nu}}$ given by

$$
\theta_{\boldsymbol{\mu}}^{\boldsymbol{\lambda}}(x)=x y_{n+g_{0}} \ldots y_{n+g_{z}} e_{\mu}^{\boldsymbol{\lambda}}, \quad \text { for } x \in S^{\boldsymbol{\nu}},
$$

where $e_{\boldsymbol{\mu}}^{\boldsymbol{\lambda}}=\sum_{\mathbf{j} \in I^{n}} e\left(j_{1}, \ldots, j_{n}, i_{1}, \ldots, i_{\gamma}\right)$ and $i_{h}=\operatorname{res}_{\mathfrak{t}_{\boldsymbol{\lambda}}^{\nu}}(n+h)$ for $1 \leq h \leq \gamma$. By definition, $\theta_{\boldsymbol{\mu}}^{\boldsymbol{\lambda}}$ is an $\mathscr{R}_{n}^{\Lambda}$-module endomorphism of $S^{\boldsymbol{\nu}}$.

3.30. Lemma. Suppose that $(\boldsymbol{\lambda}, \boldsymbol{\mu})$ is a cyclotomic Carter-Payne pair. Then $\boldsymbol{\theta}_{\boldsymbol{\mu}}^{\boldsymbol{\lambda}}$ depends only on $\boldsymbol{\lambda}, \boldsymbol{\mu}$ and $\mathfrak{t}_{\boldsymbol{\lambda}}^{\nu}$.

Proof. The tableau $\mathfrak{t}_{\boldsymbol{\lambda}}^{\nu}$ determines the idempotent $e_{\boldsymbol{\mu}}^{\boldsymbol{\lambda}}$. For $1 \leq g \leq \gamma$ let $i_{g}=$ $\operatorname{res}_{t_{\lambda}^{\nu}}(n+g)$. If $x \in S^{\nu}$ then it is easy to see that

$$
\theta_{\boldsymbol{\mu}}^{\boldsymbol{\lambda}}(x)=x \prod_{g=1}^{\gamma} y_{n+g}^{c_{g}} e_{\boldsymbol{\mu}}^{\boldsymbol{\lambda}},
$$

where $c_{g}$ is equal to the number of removable $j$-strips $C$ of length at most $\gamma_{j}$ such that $j=i_{g}-|C|+1 \in J^{*}$ and $\operatorname{row}_{t_{\lambda}^{\nu}}(n+g)=\operatorname{row} \alpha_{\boldsymbol{\lambda}}^{\nu}\left(i_{g}\right)<\operatorname{row} C \leq \operatorname{row} \alpha_{\mu}^{\nu}\left(i_{g}\right)$. (The condition $i_{g}=j+|C|-1$ simply says that the rightmost node in $C$ has residue $i_{g}$.) This implies the result. 
To show that $\theta_{\boldsymbol{\mu}}^{\boldsymbol{\lambda}}$ induces a non-zero homomorphism from $S^{\boldsymbol{\lambda}}\langle a-b+2 c-d\rangle$ to $S^{\mu}$ we need some more notation. For $0 \leq k \leq z+1$ define multipartitions $\boldsymbol{\sigma}_{k}=\operatorname{Shape}\left(\left(\mathfrak{t}_{k}\right)_{\downarrow n}\right)$ and define permutations $w_{k} \in \mathfrak{S}_{n}$ by $\left(\mathfrak{t}_{k}\right)_{\downarrow n}=\left(\mathfrak{t}_{\boldsymbol{\sigma}_{k}}^{\nu}\right)_{\downarrow n} w_{k}$. Then $\mathfrak{t}_{z+1} \triangleright \mathfrak{t}_{z} \triangleright \cdots \triangleright \mathfrak{t}_{1} \triangleright \mathfrak{t}_{0}=\mathfrak{t}_{\boldsymbol{\lambda}}^{\nu}$. Exactly as in (3.15). define integers $l_{h}^{(k)}$ and $m_{h}^{(k)}$, for $f_{k} \leq h \leq g_{k}$ and $0 \leq k \leq z$ and hence define $\psi_{w_{k}}$ as in (3.18)

3.31. Example. As in Example 3.27, let $e=0$ and let $\kappa=(0,4,0,0,4)$. Consider $\boldsymbol{\lambda}=(\emptyset|\emptyset| 2|3| 1)$ and $\boldsymbol{\mu}=(2|1| 3|\emptyset| \emptyset)$. The residues in $\boldsymbol{\nu}=\boldsymbol{\lambda} \cup \boldsymbol{\mu}=(2|1| 3|3| 1)$ are

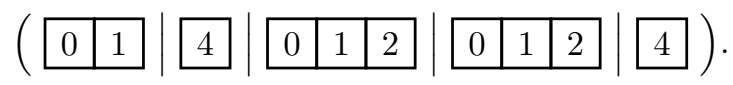

By definition, $\gamma=|\boldsymbol{\nu} \backslash \boldsymbol{\lambda}|=4$ and $z=2$. The tableaux $\mathfrak{t}_{k}$, for $0 \leq k \leq z+1$ are

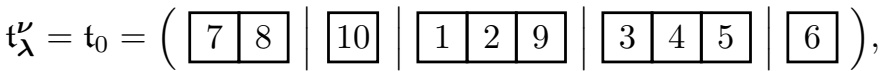

$$
\begin{aligned}
& \mathfrak{t}_{1}=\left(\begin{array}{|l|l|}
\hline 1 & 2 \\
\hline & 10
\end{array}\left|\begin{array}{|l|l|l|l|l|l|l|l|}
\hline 7 & 8 & 9 \\
\hline
\end{array}\right| \begin{array}{lll}
3 & 4 & 5 \\
\hline
\end{array}\right), \\
& \mathfrak{t}_{2}=\left(\begin{array}{|l|l|l|l|l|l|l|l|l|l|l|l|}
\hline 1 & 2 \\
\hline & 6 & 8 & 9 \\
\hline
\end{array} \mid \begin{array}{lll}
3 & 4 & 5 \\
\hline
\end{array}\right),
\end{aligned}
$$

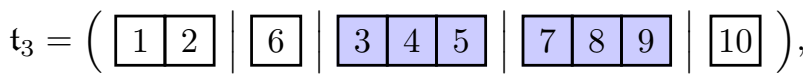

where at each step we have shaded the entries which moved. In the notation of Theorem 3.28, $a=0, b=2, c=3$ and $d=1$, so the theorem implies that there exists a non-zero homomorphism $S^{\boldsymbol{\lambda}}\langle 3\rangle \rightarrow S^{\mu}$. This map is induced by the $\mathscr{R}_{n}^{\Lambda}$ module endomorphism $\theta_{\boldsymbol{\mu}}^{\boldsymbol{\lambda}}$ of $S^{\boldsymbol{\nu}}$ which is given by $\theta_{\boldsymbol{\mu}}^{\boldsymbol{\lambda}}(x)=x y_{8} y_{9} y_{10} e_{\boldsymbol{\mu}}^{\boldsymbol{\lambda}}$, for $x \in S^{\boldsymbol{\nu}}$, where $e_{\boldsymbol{\mu}}^{\boldsymbol{\lambda}}=\sum_{\mathbf{i} \in I^{6}} e\left(i_{1}, \ldots, i_{6}, 0,1,2,4\right)$.

We now refer the reader to Lemma 3.20. It is clear that the statement given in that lemma also holds for our more generalised setup.

As in Example 3.31, there may be tableaux $\mathfrak{t}_{k}$ for $1 \leq k \leq z+1$ in which $\operatorname{row}_{\mathfrak{t}_{k}}(n+f)>\operatorname{row}_{\mathfrak{t}_{k}}(n+g)$ for some $1 \leq f<g \leq \gamma$. As a consequence, certain words in $\psi_{1}, \ldots, \psi_{n+\gamma-1}$ which previously corresponded to reduced expressions in $s_{1}, \ldots, s_{n+\gamma-1}$ no longer necessarily have this property. As we shall see, however, this will ultimately not cause us any difficulties.

3.32. Example. We continue Example 3.27 (and Example 3.31). The braid diagrams below show that $v_{\mathfrak{t}_{1}} y_{10}=v_{\mathfrak{t}_{2}}$ :
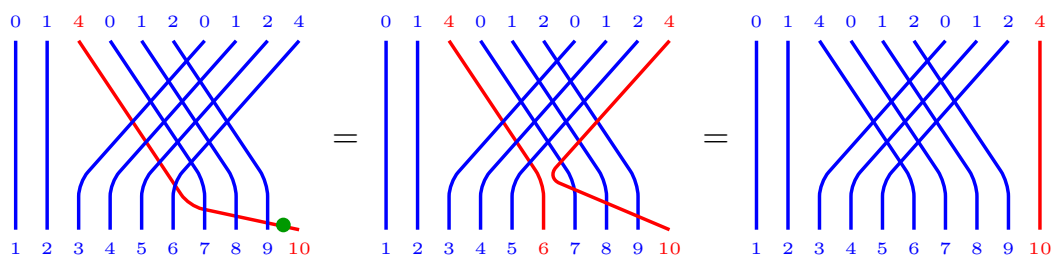

3.33. Lemma. Suppose that $\varpi$ is a word in $\mathbf{\Psi}_{n+\gamma}$ and let $B_{\varpi}$ be the braid diagram for $v_{\mathfrak{t}^{\nu}} \varpi$. Suppose that $B_{\varpi}$ has the following properties:

- If $1 \leq m<m^{\prime} \leq n$, the $m$-string and the $m^{\prime}$-string do not cross each other.

- If $1 \leq m \leq n$ and $1 \leq g \leq \gamma$, the $m$-string and the $n+g$-string cross at most once. 
- If $1 \leq g, h \leq \gamma$ and the $n+g$-string and the $n+h$-string cross, then $i_{g} \neq i_{h} \pm 1$. Let $s_{j_{1}} s_{j_{2}} \ldots s_{j_{l}}$ be a reduced expression for $\pi_{\varpi}$. Then $v_{\mathfrak{t}^{\nu} \varpi} \varpi v_{\mathfrak{t}^{\nu}} \psi_{j_{1}} \psi_{j_{2}} \ldots \psi_{j_{l}}$.

Proof. Suppose $\varpi=\psi_{k_{1}} \psi_{k_{2}} \ldots \psi_{k_{l^{\prime}}}$. As permutations, we may get from (the possibly non-reduced expression) $s_{k_{1}} s_{k_{2}} \ldots s_{k_{l^{\prime}}}$ to any reduced expression for $\pi_{\varpi}$ by applying the braid relations and the relation $s_{j}^{2}=1$ for $1 \leq j<n+\gamma$. The result follows, once we have checked that when making the corresponding manipulations of the diagram $B_{\varpi}$, we never get local diagrams of the form

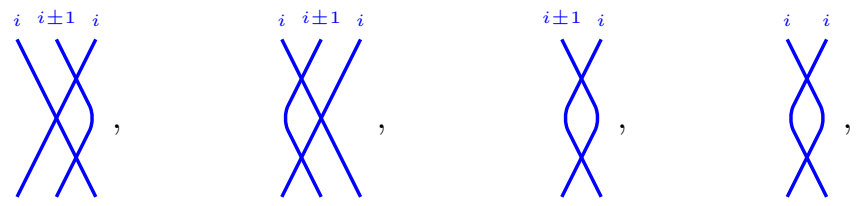

but this follows easily from our assumptions on $B_{\varpi}$.

As in (3.18) set $v_{\mathfrak{t}_{k}}=v_{\mathfrak{t}_{\boldsymbol{\sigma}_{k}}^{\nu}} \psi_{w_{k}}$, for $0 \leq k \leq z+1$, and consider the generalisation of Proposition 3.21 to the current setting. We claim that if $0 \leq k \leq z$ then

$$
v_{\mathfrak{t}_{k}} y_{n+g_{k}}=v_{\mathfrak{t}_{k+1}}+\sum_{\mathfrak{s} \in \operatorname{Std}(\boldsymbol{\nu})} a_{\mathfrak{s}} v_{\mathfrak{s}},
$$

where $a_{\mathfrak{s}} \neq 0$ only if $\operatorname{res}(\mathfrak{s})=\operatorname{res}\left(\mathfrak{t}_{\boldsymbol{\lambda}}^{\nu}\right), \operatorname{row}_{\mathfrak{s}}\left(n+f_{k}\right) \geq \operatorname{row}_{\mathfrak{t}_{k+1}}\left(n+f_{k}\right)$ and either there exists $f_{k} \leq g \leq g_{k}$ with $\operatorname{row}_{\mathfrak{s}}(n+g)>\operatorname{row}_{\mathfrak{t}_{k+1}}(n+g)$ or $\mathfrak{t}_{z+1} \not \mathfrak{s}$ (or both).

To show that (3.34) holds, we follow the proof of Proposition 3.21 and write $v_{\mathfrak{t}_{k}} y_{n+g_{k}}=\sum_{\mathfrak{u} \in \operatorname{Std}(\boldsymbol{\nu})} b_{\mathfrak{u}} v_{\mathfrak{u}}$ where $b_{\mathfrak{u}} \neq 0$ only if $\operatorname{res}(\mathfrak{u})=\operatorname{res}\left(\mathfrak{t}_{\boldsymbol{\lambda}}^{\nu}\right)$ and $\operatorname{row}_{\mathfrak{u}}\left(n+f_{k}\right) \geq$ row $_{t_{k+1}}\left(n+f_{k}\right)$. Again, we must show that $b_{\mathfrak{t}_{k+1}}=1$ and that if $\mathfrak{u} \neq \mathfrak{t}_{k+1}$ and $b_{\mathfrak{u}} \neq 0$ then either there exists $f_{k} \leq g \leq g_{k}$ such that $\operatorname{row}_{\mathfrak{u}}(n+g)>\operatorname{row}_{t_{k+1}}(n+g)$ or $\mathfrak{t}_{z+1} \unrhd \mathfrak{u}$ (or both).

Using Lemma 3.10, $v_{\mathfrak{t}_{\boldsymbol{\sigma}_{k}}^{\nu}} y_{n+g_{k}}$ is equal to the sum of diagrams of the form below, where the sum is over all integer sequences $\mathbf{m}=\left(m_{f_{k}}, \ldots, m_{g_{k}}\right)$ such that $1 \leq$ $m_{f_{k}}<m_{f_{k}+1}<\ldots<m_{g_{k}} \leq n, \operatorname{res}_{\boldsymbol{t}_{\boldsymbol{\sigma}_{k}}^{\nu}}\left(m_{h}\right)=\operatorname{res}_{\boldsymbol{t}_{\boldsymbol{\sigma}_{k}}^{\nu}}(n+h)$ and $\operatorname{row}_{\boldsymbol{t}_{\boldsymbol{\sigma}_{k}}^{\nu}}\left(m_{h}\right)>$ row $_{\boldsymbol{t}_{\boldsymbol{\sigma}_{k}}^{\nu}}(n+h)$, for $f_{k} \leq h \leq g_{k}$.

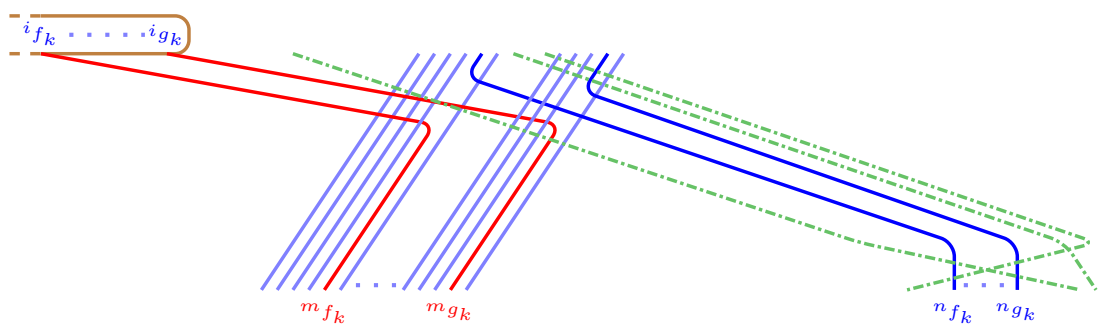

In all of these diagrams the $(n+h)$-strings, for $f_{k} \leq h \leq g_{k}$, correspond to rows below row ${\text { to } \boldsymbol{\sigma}_{k}}\left(n+f_{k}\right)$. Fix $l$ with $1 \leq l<f_{k}$ or $g_{k}<l \leq \gamma$. The dashed lines in the diagram indicate that the $(n+l)$-string is in exactly the same position as it is in the diagram for $v_{\mathfrak{t}_{\boldsymbol{\sigma}_{k}}^{\nu}}$. The $(n+l)$-string plays no role in the argument below because, by assumption, the residue of this string is not equal to the residue of an $(n+h)$-string, where $f_{k} \leq h \leq g_{k}$.

For each such $\mathbf{m}=\left(m_{f_{k}}, \ldots, m_{g_{k}}\right)$, define $\varpi_{\mathbf{m}} \in \mathcal{W}_{n+\gamma}$ and $B_{\mathbf{m}}$ as in Proposi3.21. First consider the case when $m_{h}=m_{h}^{(k)}$ for $f_{k} \leq h \leq g_{k}$. Then, consider as 
a word,

$$
\varpi_{\mathbf{m}}=\varpi \prod_{h=f_{k}}^{g_{k}} \psi_{s\left(l_{f_{k}+g_{k}-h}^{(k)}, m_{f_{k}+g_{k}-h}^{(k)}\right)}
$$

where $\varpi$ satisfies the assumptions of Lemma 3.33. Furthermore $\mathfrak{t}^{\nu} \pi_{\varpi}=v_{\boldsymbol{t}_{\boldsymbol{\sigma}_{k+1}}}$, so that $v_{\mathfrak{t}^{\nu}} \varpi=v_{\mathfrak{t}_{\sigma_{k+1}}}$, regardless of the choice of reduced expression that was used to define $v_{\mathfrak{t}_{\boldsymbol{\sigma}_{k+1}}^{\nu}}$. Therefore, as in the proof of Proposition 3.21, we obtain

$$
v_{\mathfrak{t}^{\nu}} \varpi_{\mathbf{m}} \psi_{w_{k}}=v_{\mathfrak{t}_{\boldsymbol{\sigma}_{k+1}}^{\nu}} \prod_{h=f_{k}}^{g_{k}} \psi_{s\left(l_{f_{k}+g_{k}-h}^{(k)}, m_{f_{k}+g_{k}-h}^{(k)}\right)} \psi_{w_{k}}=v_{\mathfrak{t}_{k+1}} .
$$

Now suppose that there exists $f_{k} \leq g \leq g_{k}$ such that $m_{g}>m_{g}^{(k)}$. Exactly as in the proof of Proposition 3.21, $\mathfrak{t}^{\nu} \varpi_{m} \psi_{k}$ is a linear combination of terms $v_{\mathfrak{u}}$ where $\mathfrak{u} \in \operatorname{Std}(\boldsymbol{\nu})$ is such that $\operatorname{row}_{\mathfrak{u}}(n+g)>\operatorname{row}_{\mathfrak{t}_{k+1}}(n+g)$.

Finally, suppose that $m_{h} \leq m_{h}^{(k)}$, for $1 \leq h \leq g_{k}$, and that there exists an integer $g$ such that $f_{k} \leq g \leq g_{k}$ and $m_{g}<m_{g}^{(k)}$. As in the case where $m_{h}=m_{h}^{(k)}$ for all $h$, we use Lemma 3.33 to write $v_{\mathfrak{t}^{\nu}} \varpi_{\mathbf{m}}=v_{\mathfrak{t}^{\nu}} \psi_{d\left(\pi_{\varpi_{\mathbf{m}}}\right)}$. The argument used in the proof of Proposition 3.21 shows that $v_{\mathbf{t}^{\nu}} \varpi_{\mathbf{m}} \psi_{w_{k}}$ is a linear combination of terms $v_{\mathfrak{u}}$ where $\mathfrak{u} \in \operatorname{Std}(\boldsymbol{\nu})$ and $\mathfrak{t}_{z+1} \not \mathfrak{u}$. This completes the proof of (3.34)

This establishes the required generalisation of Lemma 3.20 and Proposition 3.21. Now the argument used to prove Theorem 3.12 can be repeated, word for word, to complete the proof of Theorem 3.28.

3.5. Maps involving many cyclotomic Carter-Payne pairs. In this last section we give two applications of Theorem 3.28 to situations which involve more than one cyclotomic Carter-Payne pair. Again, we assume that $e \in\{0,2,3,4,5, \ldots\}$ is arbitrary.

If $(\boldsymbol{\lambda}, \boldsymbol{\mu})$ is a cyclotomic Carter-Payne pair, define

$$
\delta_{\boldsymbol{\mu}}^{\boldsymbol{\lambda}}=a-b+2 c-d,
$$

where $a, b, c$ and $d$ are as in Theorem 3.28, and let $\Theta_{\boldsymbol{\mu}}^{\boldsymbol{\lambda}} \in \operatorname{Hom}_{\mathscr{R}_{n}^{\Lambda}}\left(S^{\boldsymbol{\lambda}}\left\langle\delta_{\boldsymbol{\mu}}^{\boldsymbol{\lambda}}\right\rangle, S^{\boldsymbol{\mu}}\right)$ be the $\mathscr{R}_{n}^{\Lambda}$-module homomorphism induced by the map $\theta_{\boldsymbol{\mu}}^{\boldsymbol{\lambda}}$ which was defined in (3.29).

The first result in this section is almost implicit in the proof of Theorem 3.28. In the statement of the next result we adopt the convention that if $X$ and $Y$ are sets then $X \backslash Y=\{x \in X \mid x \notin Y\}$.

3.35. Theorem. Suppose that $\boldsymbol{\lambda}, \boldsymbol{\mu}, \boldsymbol{\rho} \in \mathscr{P}_{n}^{\Lambda}$ are multipartitions of $n$ such that $\boldsymbol{\lambda} \backslash \boldsymbol{\rho}=\boldsymbol{\mu} \backslash \boldsymbol{\rho}$ and $(\boldsymbol{\lambda}, \boldsymbol{\rho})$ and $(\boldsymbol{\rho}, \boldsymbol{\mu})$ are cyclotomic Carter-Payne pairs. Then $(\boldsymbol{\lambda}, \boldsymbol{\mu})$ is a cyclotomic Carter-Payne pair, $\delta_{\boldsymbol{\mu}}^{\boldsymbol{\lambda}}=\delta_{\boldsymbol{\rho}}^{\boldsymbol{\lambda}}+\delta_{\boldsymbol{\mu}}^{\boldsymbol{\rho}} \geq 2$ and there exist maps $\Theta_{\boldsymbol{\rho}}^{\boldsymbol{\lambda}} \in$ $\operatorname{Hom}_{\mathscr{R}_{n}^{\Lambda}}\left(S^{\boldsymbol{\lambda}}\left\langle\delta_{\boldsymbol{\rho}}^{\boldsymbol{\lambda}}\right\rangle, S^{\boldsymbol{\rho}}\right)$ and $\Theta_{\boldsymbol{\mu}}^{\boldsymbol{\rho}} \in \operatorname{Hom}_{\mathscr{R}_{n}^{\Lambda}}\left(S^{\boldsymbol{\rho}}\left\langle\delta_{\boldsymbol{\mu}}^{\boldsymbol{\rho}}\right\rangle, S^{\boldsymbol{\mu}}\right)$ such that

$$
0 \neq \Theta_{\boldsymbol{\mu}}^{\boldsymbol{\lambda}}=\Theta_{\boldsymbol{\mu}}^{\rho} \circ \Theta_{\boldsymbol{\rho}}^{\boldsymbol{\lambda}} \in \operatorname{Hom}_{\mathscr{R}_{n}^{\Lambda}}\left(S^{\boldsymbol{\lambda}}\left\langle\delta_{\boldsymbol{\mu}}^{\boldsymbol{\lambda}}\right\rangle, S^{\boldsymbol{\mu}}\right) .
$$

Before we prove the theorem we give an example to illustrate it.

3.36. Example. Suppose that $e=5$ and $\kappa=(4,1,0,0,2)$ and let

$$
\boldsymbol{\lambda}=(6,4,1|3,1| 1|1| 4,3), \quad \boldsymbol{\rho}=(7,5,2|1,1| 1|\emptyset| 4,3) \quad \text { and } \quad \boldsymbol{\mu}=(7,5,2|3,1| 1|1| 3,1) .
$$

Then $(\boldsymbol{\lambda}, \boldsymbol{\rho})$ and $(\boldsymbol{\rho}, \boldsymbol{\mu})$ are cyclotomic Carter-Payne pairs, so Theorem 3.35implies that $(\boldsymbol{\lambda}, \boldsymbol{\mu})$ is also a cyclotomic Carter-Payne pair, which is easily checked. The 
residue diagram of $\boldsymbol{\nu}=\boldsymbol{\lambda} \cup \boldsymbol{\mu}(=\boldsymbol{\lambda} \cup \boldsymbol{\rho}=\boldsymbol{\rho} \cup \boldsymbol{\mu})=(7,5,2|3,1| 1|1| 4,3)$ is given by

$\left(\begin{array}{|l|l|l|l|l|l|l|}\hline 4 & 0 & 1 & 2 & 3 & 4 & 0 \\ \hline 3 & 4 & 0 & 1 & 2 & \multicolumn{1}{|c}{} \\\right.$\cline { 1 - 3 } 2 & 3 & \multicolumn{7}{|c}{} \\ \cline { 1 - 2 } & \end{array}
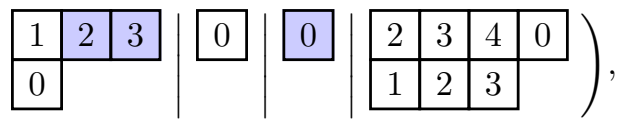

where we have shaded the nodes in $\boldsymbol{\lambda} \backslash \boldsymbol{\rho}=\boldsymbol{\mu} \backslash \boldsymbol{\rho}$. Let

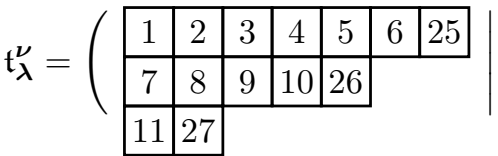

$$
\begin{aligned}
& \left.\begin{array}{|l|l|l|l|l|l|l|l|l|l|}
\hline 12 & 13 & 14 \\
\hline 15 & & 16 & 18 & 19 & 20 & 21 \\
\hline 22 & 23 & 24 &
\end{array}\right) \text { and }
\end{aligned}
$$

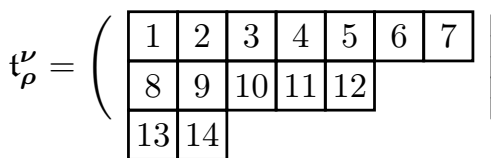
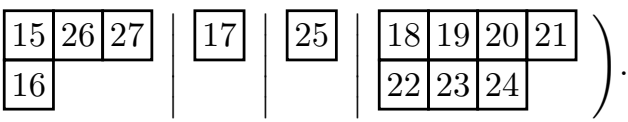

Then the $\mathscr{R}_{n}^{\Lambda}$-module endomorphisms $\theta_{\boldsymbol{\mu}}^{\boldsymbol{\lambda}}, \theta_{\boldsymbol{\rho}}^{\boldsymbol{\lambda}}$ and $\theta_{\boldsymbol{\mu}}^{\boldsymbol{\rho}}$ are given by

$$
\theta_{\boldsymbol{\mu}}^{\boldsymbol{\lambda}}(x)=x y_{25}^{4} y_{26} y_{27} e_{\boldsymbol{\mu}}^{\boldsymbol{\lambda}}, \quad \theta_{\boldsymbol{\rho}}^{\boldsymbol{\lambda}}(x)=x y_{25}^{3} y_{26} y_{27} e_{\boldsymbol{\rho}}^{\boldsymbol{\lambda}}, \quad \text { and } \quad \theta_{\boldsymbol{\mu}}^{\boldsymbol{\rho}}(x)=x y_{25} y_{27} e_{\boldsymbol{\mu}}^{\boldsymbol{\rho}},
$$

respectively, for all $x \in S^{\nu}$ and where

$$
e_{\boldsymbol{\mu}}^{\boldsymbol{\lambda}}=e_{\boldsymbol{\rho}}^{\boldsymbol{\lambda}}=e_{\boldsymbol{\mu}}^{\boldsymbol{\rho}}=\sum_{\mathbf{j} \in I^{24}} e\left(j_{1}, \ldots, j_{24}, 0,2,3\right) .
$$

So, $\theta_{\boldsymbol{\mu}}^{\boldsymbol{\rho}} \circ \theta_{\boldsymbol{\rho}}^{\boldsymbol{\lambda}}=\theta_{\boldsymbol{\mu}}^{\boldsymbol{\lambda}} \neq 0$ and it induces a non-zero $\mathscr{R}_{n}^{\Lambda}$-homomorphism $S^{\boldsymbol{\lambda}}\langle 7\rangle \rightarrow S^{\boldsymbol{\mu}}$ as claimed.

Proof of Theorem 3.35. The condition $\boldsymbol{\lambda} \backslash \boldsymbol{\rho}=\boldsymbol{\mu} \backslash \boldsymbol{\rho}$ implies that $\boldsymbol{\nu}=\boldsymbol{\lambda} \cup \boldsymbol{\rho}=\boldsymbol{\rho} \cup \boldsymbol{\mu}=$ $\boldsymbol{\lambda} \cup \boldsymbol{\mu}$ and, consequently, that $\operatorname{res}(\boldsymbol{\nu} \backslash \boldsymbol{\lambda})=\operatorname{res}(\boldsymbol{\nu} \backslash \boldsymbol{\rho})=\operatorname{res}(\boldsymbol{\nu} \backslash \boldsymbol{\mu})$ since $(\boldsymbol{\lambda}, \boldsymbol{\rho})$ and $(\boldsymbol{\rho}, \boldsymbol{\mu})$ are cyclotomic Carter-Payne pairs. Let $\gamma=|\boldsymbol{\nu} \backslash \boldsymbol{\lambda}|$ and $J=\operatorname{res}(\boldsymbol{\nu} \backslash \boldsymbol{\lambda})$ and note that if $j \leq_{J} i$ then $\operatorname{row} \alpha_{\boldsymbol{\rho}}^{\nu}(i)=\operatorname{row} \alpha_{\boldsymbol{\rho}}^{\nu}(j)$ and $\operatorname{row} \alpha_{\boldsymbol{\mu}}^{\nu}(i)=\operatorname{row} \alpha_{\boldsymbol{\mu}}^{\nu}(j)$. It follows that $(\boldsymbol{\lambda}, \boldsymbol{\mu})$ is also a cyclotomic Carter-Payne pair. By Theorem 3.28 there exist non-zero maps $\Theta_{\boldsymbol{\mu}}^{\boldsymbol{\lambda}}, \Theta_{\boldsymbol{\rho}}^{\boldsymbol{\lambda}}$ and $\Theta_{\boldsymbol{\mu}}^{\rho}$, as in the statement of the theorem, and it remains to show that $\Theta_{\mu}^{\lambda}=\Theta_{\mu}^{\rho} \circ \Theta_{\rho}^{\lambda}$.

As in the proof of Theorem 3.28, let $\mathfrak{t}_{\boldsymbol{\lambda}}^{\nu}$ be a standard $\boldsymbol{\nu}$-tableau such that $\left(\mathfrak{t}_{\boldsymbol{\lambda}}^{\nu}\right)_{\downarrow n}=\mathfrak{t}^{\boldsymbol{\lambda}}$ and $\mathfrak{t}_{\boldsymbol{\lambda}}^{\nu}\left(\alpha_{\boldsymbol{\lambda}}^{\boldsymbol{\nu}}(i+1)\right)=\mathfrak{t}_{\boldsymbol{\lambda}}^{\nu}\left(\alpha_{\boldsymbol{\lambda}}^{\nu}(i)\right)+1$, for $i, i+1 \in J$. Let $\mathfrak{t}_{\boldsymbol{\rho}}^{\boldsymbol{\nu}}$ be the unique standard $\boldsymbol{\nu}$-tableau such that $\mathfrak{t}_{\rho}^{\nu} \in \operatorname{Std}_{\boldsymbol{\lambda}}^{e}(\boldsymbol{\nu})$ and $\left(\mathfrak{t}_{\boldsymbol{\rho}}^{\nu}\right)_{\downarrow n}=\mathfrak{t}^{\rho}$ and define $\mathfrak{t}_{\boldsymbol{\mu}}^{\nu}$ similarly. Using these tableau, define maps $\theta_{\boldsymbol{\mu}}^{\boldsymbol{\lambda}}, \theta_{\boldsymbol{\rho}}^{\boldsymbol{\lambda}}$ and $\theta_{\boldsymbol{\mu}}^{\boldsymbol{\rho}}$ as in (3.29). Then these three maps are $\mathscr{R}_{n}^{\Lambda}$-module endomorphisms of $S^{\nu}$ and by Lemma 3.30 they depend only on the set of removable $j$-strips which occur between $\boldsymbol{\nu} \backslash \boldsymbol{\lambda}$ and $\boldsymbol{\nu} \backslash \boldsymbol{\mu}$. Consequently, $\theta_{\boldsymbol{\mu}}^{\boldsymbol{\lambda}}=\theta_{\boldsymbol{\mu}}^{\boldsymbol{\rho}} \circ \theta_{\boldsymbol{\rho}}^{\boldsymbol{\lambda}}$ as endomorphisms of $S^{\boldsymbol{\nu}}$. By the same reasoning, $\delta_{\boldsymbol{\mu}}^{\boldsymbol{\lambda}}=\delta_{\boldsymbol{\rho}}^{\boldsymbol{\lambda}}+\delta_{\boldsymbol{\mu}}^{\rho}$.

By the proof of Theorem 3.28, the map $\theta_{\boldsymbol{\mu}}^{\rho} \circ \theta_{\boldsymbol{\rho}}^{\boldsymbol{\lambda}}$ sends $\check{S}_{\mathfrak{t}_{\lambda}^{\nu}}^{\nu}$ into $\hat{S}_{\mathfrak{t}_{\mu}^{\nu}}^{\nu}$. Therefore, $\theta_{\boldsymbol{\mu}}^{\boldsymbol{\rho}} \circ \theta_{\boldsymbol{\rho}}^{\boldsymbol{\lambda}}$ induces an $\mathscr{R}_{n}^{\Lambda}$-module homomorphism from $S^{\boldsymbol{\lambda}}\left\langle\delta_{\boldsymbol{\mu}}^{\boldsymbol{\lambda}}\right\rangle$ to $S^{\boldsymbol{\mu}}$ by Lemma 2.24. As $\theta_{\boldsymbol{\mu}}^{\boldsymbol{\lambda}}=\theta_{\boldsymbol{\mu}}^{\boldsymbol{\rho}} \circ \theta_{\boldsymbol{\rho}}^{\boldsymbol{\lambda}}$, it follows that $\Theta_{\boldsymbol{\mu}}^{\boldsymbol{\lambda}}=\Theta_{\boldsymbol{\mu}}^{\boldsymbol{\rho}} \circ \Theta_{\boldsymbol{\rho}}^{\boldsymbol{\lambda}}$. In particular, the map $\Theta_{\boldsymbol{\mu}}^{\boldsymbol{\rho}} \circ \Theta_{\boldsymbol{\rho}}^{\boldsymbol{\lambda}}$ is a non-zero homomorphism in $\operatorname{Hom}_{\mathscr{R}_{n}^{\Lambda}}\left(S^{\boldsymbol{\lambda}}\left\langle\delta_{\boldsymbol{\mu}}^{\boldsymbol{\lambda}}\right\rangle, S^{\boldsymbol{\mu}}\right)$.

Before stating the second result in this section we introduce some new notation. Suppose that $\boldsymbol{\lambda}=\left(\lambda^{(1)}, \ldots, \lambda^{(\ell)}\right) \in \mathscr{P}_{n}^{\Lambda}$ and recall that $\lambda^{(k)}=\left(\lambda_{1}^{(k)}, \lambda_{2}^{(k)}, \ldots\right)$, 
for $1 \leq k \leq \ell$. Fix a row index $r=(l, a)$. Then $r$ determines the row slice $\boldsymbol{\lambda}=\boldsymbol{\lambda}^{[1]} \sqcup \boldsymbol{\lambda}^{[2]}$ of $\boldsymbol{\lambda}$, where

$$
\begin{aligned}
& \lambda^{[1]}=\left(\lambda^{(1)}, \ldots, \lambda^{(l-1)},\left(\lambda_{1}^{(l)}, \ldots, \lambda_{a}^{(l)}\right)\right), \\
& \left.\lambda^{[2]}=\left(\lambda_{a+1}^{(l)}, \lambda_{a+2}^{(l)}, \ldots\right), \lambda^{(l+1)}, \ldots, \lambda^{(\ell)}\right) .
\end{aligned}
$$

Define $n_{1}=\left|\boldsymbol{\lambda}^{[1]}\right|, n_{2}=\left|\boldsymbol{\lambda}^{[2]}\right|, l_{1}=l$ and $l_{2}=\ell-l+1$, for $b=1,2$. We consider the $l_{b}$-multipartition $\lambda^{[b]}$ to be an element of $\mathscr{P}_{n_{b}}^{\Lambda_{b}}$, where the weight $\Lambda_{b} \in P^{+}$is determined by the multisets of residues of the nodes in $\lambda^{[b]}$, which we identify with a subset of $\boldsymbol{\lambda}$ in the obvious way, for $b=1,2$.

Iterating this construction, we consider row slices $\boldsymbol{\lambda}=\boldsymbol{\lambda}^{[1]} \sqcup \cdots \sqcup \boldsymbol{\lambda}^{[s]}$. We emphasize that we consider each slice $\boldsymbol{\lambda}^{[b]}$ as a subset of $\boldsymbol{\lambda}$, so $\boldsymbol{\lambda}^{[b]}$ comes equipped with a multiset of residues for $1 \leq b \leq s$.

3.37. Theorem. Suppose that $e \in\{0,2,3,4, \ldots\}$ and that $\boldsymbol{\lambda}, \boldsymbol{\mu} \in \mathscr{P}_{n}^{\Lambda}$ are multipartitions which admit row slices $\boldsymbol{\lambda}=\boldsymbol{\lambda}^{[1]} \sqcup \cdots \sqcup \boldsymbol{\lambda}^{[s]}$ and $\boldsymbol{\mu}=\boldsymbol{\mu}^{[1]} \sqcup \cdots \sqcup \boldsymbol{\mu}^{[s]}$ such that $\ell\left(\boldsymbol{\lambda}^{[b]}\right)=\ell\left(\boldsymbol{\mu}^{[b]}\right),\left|\boldsymbol{\lambda}^{[b]}\right|=\left|\boldsymbol{\mu}^{[b]}\right|$ and $\left(\boldsymbol{\lambda}^{[b]}, \boldsymbol{\mu}^{[b]}\right)$ is a cyclotomic Carter-Payne pair, for $1 \leq b \leq s$. Then

$$
\operatorname{Hom}_{\mathscr{R}_{n}^{\Lambda}}\left(S^{\boldsymbol{\lambda}}\left\langle\delta_{\boldsymbol{\mu}}^{\boldsymbol{\lambda}}\right\rangle, S^{\boldsymbol{\mu}}\right) \neq 0
$$

where $\delta_{\boldsymbol{\mu}}^{\boldsymbol{\lambda}}=\delta_{\boldsymbol{\mu}^{[1]}}^{\boldsymbol{\lambda}^{[1]}}+\cdots+\delta_{\boldsymbol{\mu}^{[s]}}^{\boldsymbol{\lambda}^{[s]}} \geq s$.

If $\Lambda=\Lambda_{0}$ is a weight of level 1 then the main result of [22] implies that

$$
\operatorname{Hom}_{\mathscr{H}_{n}^{\Lambda}}\left(\underline{S}^{\boldsymbol{\lambda}}, \underline{S}^{\boldsymbol{\mu}}\right) \cong \bigotimes_{b=1}^{s} \operatorname{Hom}_{\mathscr{H}_{n_{b}}^{\Lambda_{b}}}\left(\underline{S}^{\boldsymbol{\lambda}^{[b]}}, \underline{S}^{\boldsymbol{\mu}^{[b]}}\right)
$$

as vector spaces. Therefore, the $\mathscr{H}_{n}^{\Lambda}$-module analogue of Theorem 3.37 is a corollary of Theorem 3.28 and 22] when $\Lambda=\Lambda_{0}$. Theorem 3.37] suggests that there is a higher level analogue of the "row removal" results of [22].

3.38. Example. Let $e=3$ and let $\kappa=(0,2,0)$. Consider

$$
\begin{aligned}
& \boldsymbol{\lambda}=(5,4,1|5,3| 5)=(5,4,1 \mid 5,3) \sqcup(\emptyset \mid 5) \quad \text { and } \\
& \boldsymbol{\mu}=(6,5,1|3,3,2| 3)=(6,5,1 \mid 3,3) \sqcup(2 \mid 3) .
\end{aligned}
$$

Set $\boldsymbol{\nu}=\boldsymbol{\lambda} \cup \boldsymbol{\mu}=(6,5,1|5,3,2| 5)$. Then

$$
\boldsymbol{\nu}=\left(\begin{array}{|l|l|l|l|l|l|}
\hline 0 & 1 & 2 & 0 & 1 & 2 \\
\hline 2 & 0 & 1 & 2 & 0 & \\
\cline { 1 - 2 } 1 & \multicolumn{1}{|l}{}
\end{array}\right.
$$

\begin{tabular}{|l|l|l|l|l|}
\hline 2 & 0 & 1 & 2 & 0 \\
\hline 1 & 2 & 0 & \multicolumn{1}{|c|}{} \\
\cline { 1 - 2 } 0 & 1 &
\end{tabular}

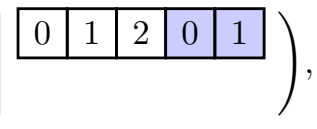

where we have shaded all of the nodes in $\boldsymbol{\nu} \backslash(\boldsymbol{\lambda} \cap \boldsymbol{\mu})$. Using the obvious notation for the row slices of $\boldsymbol{\lambda}$ and $\boldsymbol{\mu}$ at row $r=(2,2)$, we see that $\left(\boldsymbol{\lambda}^{[1]}, \boldsymbol{\mu}^{[1]}\right)$ and $\left(\boldsymbol{\lambda}^{[2]}, \boldsymbol{\mu}^{[2]}\right)$ are both cyclotomic Carter-Payne pairs. Therefore, $\operatorname{Hom}_{\mathscr{R}_{n}^{\Lambda}}\left(S^{\boldsymbol{\lambda}}\langle 5\rangle, S^{\boldsymbol{\mu}}\right) \neq 0$ by Theorem 3.37 To construct the homomorphism $\theta_{\boldsymbol{\mu}}^{\boldsymbol{\lambda}}$ in this example we would define

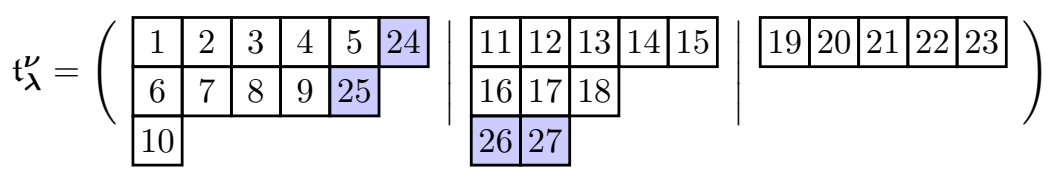

and we define $\theta_{\boldsymbol{\mu}}^{\boldsymbol{\lambda}}(x)=x y_{24} y_{25} y_{27} e_{\boldsymbol{\mu}}^{\boldsymbol{\lambda}}$. 
Sketch of the proof of Theorem 3.37. We sketch the proof only in the special case when $s=2$. The general case is similar except that it requires more notation.

Let $\boldsymbol{\nu}=\boldsymbol{\lambda} \cup \boldsymbol{\mu}=\boldsymbol{\lambda}^{[1]} \sqcup \boldsymbol{\lambda}^{[2]} \cup \boldsymbol{\mu}^{[1]} \sqcup \boldsymbol{\mu}^{[2]}$ set $\gamma=|\boldsymbol{\nu} \backslash \boldsymbol{\lambda}|=\gamma_{1}+\gamma_{2}$, where $\gamma_{1}=\left|\boldsymbol{\lambda}^{[1]} \backslash \boldsymbol{\mu}^{[1]}\right|$ and $\gamma_{2}=\left|\boldsymbol{\lambda}^{[2]} \backslash \boldsymbol{\mu}^{[2]}\right|$. As in Example 3.38, define $\mathfrak{t}_{\boldsymbol{\lambda}}^{\boldsymbol{\nu}}$ to be the standard $\boldsymbol{\nu}$-tableau such that $\left(\mathfrak{t}_{\boldsymbol{\lambda}}^{\nu}\right)_{\downarrow n}=\mathfrak{t}^{\boldsymbol{\lambda}}$, the numbers $n+1, \ldots, n+\gamma_{1}$ appear in $\boldsymbol{\nu} \backslash\left(\boldsymbol{\lambda} \cup \boldsymbol{\mu}^{[2]}\right), n+\gamma_{1}+1, \ldots, n+\gamma_{1}+\gamma_{2}$ appear in $\boldsymbol{\nu} \backslash\left(\boldsymbol{\lambda} \cup \boldsymbol{\mu}^{[1]}\right)$, and $n+1, \ldots, n+\gamma$ are entered into $\mathfrak{t}_{\boldsymbol{\lambda}}^{\nu}$ subject to the usual residue constraints.

Using the tableau $\mathfrak{t}_{\lambda}^{\nu}$ define $\mathscr{R}_{n}^{\Lambda}$-module endomorphisms $\theta^{[1]}$ and $\theta^{[2]}$ of $S^{\boldsymbol{\nu}}$ as in (3.29) Then $\theta^{[1]}$ is right multiplication by a polynomial in $y_{n+1}, \ldots, y_{n+\gamma_{1}}$ times $e_{\boldsymbol{\mu}}^{\boldsymbol{\lambda}}$, and $\theta^{[2]}$ is right multiplication by a polynomial in $y_{n+\gamma_{1}+1}, \ldots, y_{n+\gamma}$ times $e_{\boldsymbol{\mu}}^{\boldsymbol{\lambda}}$. By construction, these two maps commute, so $\theta^{[1]} \circ \theta^{[2]}=\theta^{[2]} \circ \theta^{[1]}$ is an $\mathscr{R}_{n}^{\Lambda}$-module endomorphism of $S^{\boldsymbol{\nu}}$. By Lemma 3.30, $\theta^{[1]}$ depends only on $\boldsymbol{\lambda}^{[1]}$ and $\boldsymbol{\mu}^{[1]}$ and, similarly, $\theta^{[2]}$ depends only on $\boldsymbol{\lambda}^{[2]}$ and $\boldsymbol{\mu}^{[2]}$. By Theorem 3.28, the map $\theta^{[1]}$ induces an $\mathscr{R}_{n}^{\Lambda}$-module homomorphism $\Theta^{[1]}$ from $S^{\boldsymbol{\lambda}^{[1]} \sqcup \boldsymbol{\lambda}^{[2]}}\left\langle\delta_{\boldsymbol{\mu}^{[1]}}^{\boldsymbol{\lambda}^{[1]}}\right\rangle$ to $S^{\boldsymbol{\mu}^{[1]} \sqcup \boldsymbol{\lambda}^{[2]}}$. Similarly, because $\theta^{[2]}$ depends only on $\boldsymbol{\lambda}^{[2]}$ and $\boldsymbol{\mu}^{[2]}$, the map $\theta^{[2]}$ induces an $\mathscr{R}_{n}^{\Lambda}$-module homomorphism $\Theta^{[2]}$ from $S^{\boldsymbol{\mu}^{[1]} \sqcup \boldsymbol{\lambda}^{[2]}}\left\langle\delta_{\boldsymbol{\mu}^{[2]}}^{\boldsymbol{\lambda}^{[2]}}\right\rangle$ to $S^{\boldsymbol{\mu}^{[1]} \sqcup \boldsymbol{\mu}^{[2]}}$. As in the proof of Theorem 3.35, it follows that $\theta^{[2]} \circ \theta^{[1]}$ induces the $\mathscr{R}_{n}^{\Lambda}$-module homomorphism $\Theta^{[2]} \circ \Theta^{[1]}$ from $S^{\boldsymbol{\lambda}}\left\langle\delta_{\boldsymbol{\mu}}^{\boldsymbol{\lambda}}\right\rangle$ to $S^{\boldsymbol{\mu}}$.

To complete the proof it remains to show that $\Theta^{[2]} \circ \Theta^{[1]}$ is non-zero. By construction, $\theta^{[2]}$ only ever moves entries in the tableaux which are in the rows after $\ell\left(\boldsymbol{\lambda}^{[1]}\right)=\ell\left(\boldsymbol{\mu}^{[1]}\right)$. Let $\mathfrak{t}_{0}^{[1]}, \ldots, \mathfrak{t}_{z_{1}+1}^{[1]}$ and $\mathfrak{t}_{0}^{[2]}, \ldots, \mathfrak{t}_{z_{2}+1}^{[2]}$ be the tableaux constructed during the proof of Theorem 3.28 when showing that the induced maps $\Theta^{[1]}$ and $\Theta^{[2]}$, respectively, are well-defined and non-zero. Therefore, extending the slice notation to tableaux in the obvious way, and working modulo $\hat{S}_{\boldsymbol{\mu}}^{\nu}$, the argument used to prove (3.25) shows that $\theta^{[2]} \circ \theta^{[1]}$ sends $v_{\mathfrak{t}_{\lambda}^{\nu}}$ to $v_{\mathfrak{t}_{z_{1}+1}^{[1]}}^{\left[\mathfrak{t}_{z_{2}+1}^{[2]}\right.}$ plus a linear combination of terms $v_{\mathfrak{u}}$, where $\mathfrak{u} \neq \mathfrak{t}_{z_{1}+1}^{[1]} \sqcup \mathfrak{t}_{z_{2}+1}^{[2]}$. It follows that $\Theta^{[2]} \circ \Theta^{[1]} \neq 0$.

\section{REFERENCES}

[1] Susumu Ariki, On the decomposition numbers of the Hecke algebra of $G(m, 1, n)$, J. Math. Kyoto Univ. 36 (1996), no. 4, 789-808. MR1443748(98h:20012)

[2] Susumu Ariki, Andrew Mathas, and Hebing Rui, Cyclotomic Nazarov-Wenzl algebras, Nagoya Math. J. 182 (2006), 47-134. MR2235339 (2007d:20005)

[3] Michel Broué, Reflection groups, braid groups, Hecke algebras, finite reductive groups, Current developments in mathematics, 2000, Int. Press, Somerville, MA, 2001, pp. 1-107. MR.1882533 (2003e:20044)

[4] Jonathan Brundan and Alexander Kleshchev, Blocks of cyclotomic Hecke algebras and Khovanov-Lauda algebras, Invent. Math. 178 (2009), no. 3, 451-484, DOI 10.1007/s00222009-0204-8. MR2551762(2010k:20010)

[5] _ Graded decomposition numbers for cyclotomic Hecke algebras, Adv. Math., 222 (2009), 1883-1942. http://dx.doi.org/10.1016/j.aim.2009.06.018

[6] Jonathan Brundan, Alexander Kleshchev, and Weiqiang Wang, Graded Specht modules, J. Reine Angew. Math. 655 (2011), 61-87, DOI 10.1515/CRELLE.2011.033. MR2806105 (2012f:20006)

[7] R. W. Carter and M. T. J. Payne, On homomorphisms between Weyl modules and Specht modules, Math. Proc. Cambridge Philos. Soc. 87 (1980), no. 3, 419-425, DOI 10.1017/S0305004100056851. MR556922 (81h:20048)

[8] K. Corlett, Homomorphisms Between Specht Modules of the Ariki Koike Algebra, 2011, preprint. http://arxiv.org/abs/1108.3672. 
[9] K. Corlett, On homomorphisms between Specht modules For the Ariki-Koike algebras, PhD thesis, University of East Anglia, UK, 2012.

[10] Richard Dipper, Gordon James, and Andrew Mathas, Cyclotomic q-Schur algebras, Math. Z. 229 (1998), no. 3, 385-416, DOI 10.1007/PL00004665. MR.1658581(2000a:20033)

[11] J. Dixon, Some results concerning Verma modules, PhD thesis, Queen Mary College, University of London, 2008.

[12] Craig J. Dodge, Large dimension homomorphism spaces between Specht modules for symmetric groups, J. Pure Appl. Algebra 215 (2011), no. 12, 2949-2956, DOI 10.1016/j.jpaa.2011.04.015. MR2811577(2012h:20024)

[13] Harald Ellers and John Murray, Branching rules for Specht modules, J. Algebra 307 (2007), no. 1, 278-286, DOI 10.1016/j.jalgebra.2006.07.032. MR2278054 (2007k:20007)

[14] I. Gordon and I. Losev, On category $\mathcal{O}$ for cyclotomic rational Cherednik algebras, J. Eur. Math. Soc., to appear. http://arxiv.org/abs/1109.2315.

[15] Jun $\mathrm{Hu}$ and Andrew Mathas, Graded cellular bases for the cyclotomic KhovanovLauda-Rouquier algebras of type A, Adv. Math. 225 (2010), no. 2, 598-642, DOI 10.1016/j.aim.2010.03.002. MR2671176 (2011g:20006)

[16] _ Seminormal forms and cyclotomic quiver Hecke algebras of type A, 2013, preprint. http://arxiv.org/abs/1304.0906.

[17] V. G. KAC, Infinite dimensional Lie algebras, CUP, Cambridge, third ed., 1994.

[18] Mikhail Khovanov and Aaron D. Lauda, A diagrammatic approach to categorification of quantum groups. I, Represent. Theory 13 (2009), 309-347, DOI 10.1090/S1088-4165-09-00346-X. MR2525917(2010i:17023)

[19] Mikhail Khovanov and Aaron D. Lauda, A diagrammatic approach to categorification of quantum groups II, Trans. Amer. Math. Soc. 363 (2011), no. 5, 2685-2700, DOI 10.1090/S00029947-2010-05210-9. MR2763732(2012a:17021)

[20] Alexander S. Kleshchev, Andrew Mathas, and Arun Ram, Universal graded Specht modules for cyclotomic Hecke algebras, Proc. Lond. Math. Soc. (3) 105 (2012), no. 6, 1245-1289, DOI 10.1112/plms/pds019. MR3004104

[21] Sinéad Lyle, Some q-analogues of the Carter-Payne theorem, J. Reine Angew. Math. 608 (2007), 93-121, DOI 10.1515/CRELLE.2007.054. MR2339470 (2008f:20016)

[22] Sinéad Lyle and Andrew Mathas, Row and column removal theorems for homomorphisms of Specht modules and Weyl modules, J. Algebraic Combin. 22 (2005), no. 2, 151-179, DOI 10.1007/s10801-005-2511-5. MR2164395 (2006e:20009)

[23] Sinéad Lyle and Andrew Mathas, Carter-Payne homomorphisms and Jantzen filtrations, J. Algebraic Combin. 32 (2010), no. 3, 417-457, DOI 10.1007/s10801-010-0222-z. MR2721060 (2011h:20006)

[24] Andrew Mathas, Iwahori-Hecke algebras and Schur algebras of the symmetric group, University Lecture Series, vol. 15, American Mathematical Society, Providence, RI, 1999. MR:1711316 (2001g:20006)

[25] R. Rouquier, 2-Kac-Moody algebras, 2008, preprint. http://arxiv.org/abs/0812.5023.

School of Mathematics, University of East Anglia, Norwich NR4 7TJ, United KingDOM

E-mail address: s.lyle@uea.ac.uk

School of Mathematics and Statistics, University of Sydney, NSW 2006, Australia

E-mail address: andrew.mathas@sydney.edu.au 